\title{
Measurement of the top quark pair production charge asymmetry in proton-proton collisions at $\sqrt{s}=7 \mathrm{TeV}$ using the ATLAS detector
}

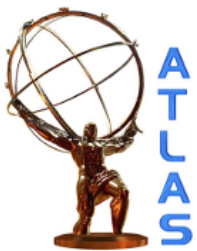

\section{The ATLAS collaboration}

E-mail: atlas.publications@cern.ch

ABSTRACT: This paper presents a measurement of the top quark pair $(t \bar{t})$ production charge asymmetry $A_{C}$ using $4.7 \mathrm{fb}^{-1}$ of proton-proton collisions at a centre-of-mass energy $\sqrt{s}=7 \mathrm{TeV}$ collected by the ATLAS detector at the LHC. A $t \bar{t}$-enriched sample of events with a single lepton (electron or muon), missing transverse momentum and at least four high transverse momentum jets, of which at least one is tagged as coming from a $b$-quark, is selected. A likelihood fit is used to reconstruct the $t \bar{t}$ event kinematics. A Bayesian unfolding procedure is employed to estimate $A_{C}$ at the parton-level. The measured value of the $t \bar{t}$ production charge asymmetry is $A_{C}=0.006 \pm 0.010$, where the uncertainty includes both the statistical and the systematic components. Differential $A_{C}$ measurements as a function of the invariant mass, the rapidity and the transverse momentum of the $t \bar{t}$ system are also presented. In addition, $A_{C}$ is measured for a subset of events with large $t \bar{t}$ velocity, where physics beyond the Standard Model could contribute. All measurements are consistent with the Standard Model predictions.

KEYwords: Hadron-Hadron Scattering, Top physics

ARXIV EPRINT: 1311.6724 


\section{Contents}

1 Introduction 1

2 Data sample, simulated samples and event selection 3

2.1 Samples 3

2.2 Event selection 3

2.3 Background estimation 5

$3 \quad$ The $t \bar{t}$ production charge asymmetry measurement $\quad 7$

$\begin{array}{lll}3.1 & \text { Reconstruction of the } t \bar{t} \text {-system } & 7\end{array}$

$\begin{array}{lll}3.2 & \text { Unfolding procedure } & 7\end{array}$

$\begin{array}{llr}3.3 & \text { Systematic uncertainties } & 9\end{array}$

4 Results $\quad 11$

4.1 Inclusive and differential measurements 11

$\begin{array}{lll}4.2 & \text { Measurements for } \beta_{z, t \bar{t}}>0.6 & 12\end{array}$

$\begin{array}{lll}4.3 & \text { Interpretation } & 13\end{array}$

$\begin{array}{llr}5 & \text { Conclusion } & 14\end{array}$

The ATLAS collaboration $\quad 22$

\section{Introduction}

The measurement of the $t \bar{t}$ production charge asymmetry represents an important test of quantum chromodynamics (QCD) at high energies and is also an ideal place to observe effects of possible new physics processes beyond the Standard Model (BSM). Several BSM processes can alter this asymmetry [1-13], either with anomalous vector or axial-vector couplings (i.e. axigluons) or via interference with the Standard Model (SM). Different models also predict different asymmetries as a function of the invariant mass $m_{t \bar{t}}$ [14], the transverse momentum $p_{\mathrm{T}, t \bar{t}}$ and the rapidity $\left|y_{t \bar{t}}\right|$ of the $t \bar{t}$-system.

At leading order $(\mathrm{LO}), t \bar{t}$ production at hadron colliders is predicted to be symmetric under the exchange of top quark and antiquark. At next-to-leading order (NLO), the process $q \bar{q} \rightarrow t \bar{t} g$ exhibits an asymmetry in the rapidity distributions of the top quark and antiquark, due to interference between initial- and final- state gluon emission. In addition, the $q \bar{q} \rightarrow t \bar{t}$ process itself possesses an asymmetry due to the interference between the Born and the NLO diagrams. The $q g$ production process is also asymmetric, but its contribution is much smaller than the $q \bar{q}$ one. The production of $t \bar{t}$ events by gluon fusion, $g g \rightarrow t \bar{t}$, is symmetric. At the Tevatron proton-antiproton collider, where $t \bar{t}$ events are predominantly produced by $q \bar{q}$ annihilation, top quarks are preferentially emitted in the direction of the 
incoming quark while the top antiquarks are emitted preferentially in the direction of the incoming antiquark [15-21]. The $t \bar{t}$ asymmetry at the Tevatron is therefore measured as a forward-backward asymmetry,

$$
A_{\mathrm{FB}}=\frac{N(\Delta y>0)-N(\Delta y<0)}{N(\Delta y>0)+N(\Delta y<0)}
$$

where $\Delta y \equiv y_{t}-y_{\bar{t}}$ is the difference in rapidity between top quarks and antiquarks, and $N$ represents the number of events with $\Delta y$ being positive or negative. The interest in this measurement has grown after $\mathrm{CDF}$ and $\mathrm{D} 0$ collaborations reported $A_{\mathrm{FB}}$ measurements significantly larger than the SM predictions, in both the inclusive and differential case as a function of $m_{t \bar{t}}$ and $\left|y_{t \bar{t}}\right|[22-26]$.

In proton-proton $(p p)$ collisions at the LHC, the dominant mechanism for $t \bar{t}$ production is the $g g$ fusion process, while production via $q \bar{q}$ or $q g$ interactions is small. Since the colliding beams are symmetric, $A_{\mathrm{FB}}$ is no longer a useful observable. However, $t \bar{t}$ production via $q \bar{q}$ or $q g$ processes is asymmetric under top quark-antiquark exchange, and, in addition, the valence quarks carry, on average, a larger momentum fraction than antiquarks from the sea. Hence for $q \bar{q}$ or $q g$ production processes at the LHC, QCD predicts a small excess of centrally produced top antiquarks while top quarks are produced, on average, at higher absolute rapidities. Therefore, the $t \bar{t}$ production charge asymmetry $A_{\mathrm{C}}$ is defined as $[1,27]$

$$
A_{\mathrm{C}}=\frac{N(\Delta|y|>0)-N(\Delta|y|<0)}{N(\Delta|y|>0)+N(\Delta|y|<0)}
$$

where $\Delta|y| \equiv\left|y_{t}\right|-\left|y_{\bar{t}}\right|$ is the difference between the absolute value of the top quark rapidity $\left|y_{t}\right|$ and the absolute value of the top antiquark rapidity $\left|y_{\bar{t}}\right|$.

The SM prediction for the $t \bar{t}$ production charge asymmetry at the LHC is $A_{\mathrm{C}}^{\mathrm{SM}}=$ $0.0123 \pm 0.0005[21]$, computed at NLO in QCD including electroweak corrections. Recent asymmetry measurements at the LHC [28-30] did not report any significant deviation from the SM predictions in either the inclusive or differential $A_{\mathrm{C}}$ measurements. Agreement with the SM $A_{\mathrm{C}}$ predictions at the LHC is compatible with the larger than expected $A_{\mathrm{FB}}$ values measured at the Tevatron for the most general new physics scenarios [31], but creates a tension between the measurements at the two colliders in specific simple models [8]. This motivates the interest in a more precise measurement of the $t \bar{t}$ production charge asymmetry.

In this paper, a measurement of the $t \bar{t}$ production charge asymmetry in the singlelepton final state is reported. To allow comparisons with theory calculations, a Bayesian unfolding procedure is applied to account for distortions due to acceptance and detector effects, leading to parton-level $A_{\mathrm{C}}$ measurements. Compared with the previous $t \bar{t}$ production charge asymmetry measurement performed by the ATLAS experiment and described in ref. [30], the full 2011 data sample is now used and new differential $A_{\mathrm{C}}$ measurements are performed. In particular, an inclusive $A_{\mathrm{C}}$ measurement and measurements of $A_{\mathrm{C}}$ as a function of $m_{t \bar{t}}, p_{\mathrm{T}, t \bar{t}}$ and $\left|y_{t \bar{t}}\right|$ are presented. The inclusive $A_{\mathrm{C}}$ result and the differential result as a function of $m_{t \bar{t}}$ are also presented with the additional requirement of a minimum velocity $\beta_{z, t \bar{t}}$ of the $t \bar{t}$-system along the beam axis to enhance the sensitivity to BSM effects [32]. 


\section{Data sample, simulated samples and event selection}

\subsection{Samples}

The measurement is performed using $7 \mathrm{TeV} p p$ collisions recorded by the ATLAS detector [33] at the LHC during 2011. The ATLAS detector is composed of inner tracking detectors immersed in a $2 \mathrm{~T}$ axial magnetic field provided by a solenoid, surrounded by calorimeters and, as an outer layer, by a muon spectrometer in a magnetic field provided by three large air-core toroid magnet systems. ${ }^{1}$ After applying detector and data-quality requirements, the recorded data corresponds to an integrated luminosity of $4.7 \mathrm{fb}^{-1}$ [34].

Simulated $t \bar{t}$ events are modelled using the LO multi-parton matrix-element Monte Carlo (MC) generator ALPGEN [35] with the LO CTEQ6L1 [36] parton distribution function (PDF) for the proton. Parton showering and the underlying event are modelled using HERWIG [37] and JIMMY [38] with the AUET2 parameter settings [39]. The $t \bar{t}$ sample is generated assuming a top quark mass of $172.5 \mathrm{GeV}$ and it is normalised to a total inclusive cross-section of $177_{-11}^{+10} \mathrm{pb}$ computed at next-to-next-to-leading-order (NNLO) in QCD including resummation of next-to-next-to-leading-logarithmic (NNLL) soft gluon terms with Top ++2.0 [40-45]. The uncertainties included in the calculation are those related to the choice of the PDF set (following the PDF4LHC prescriptions [46]), the variations of $\alpha_{S}$ and the choice of renormalisation and factorisation scales. These uncertainties are added in quadrature to give the quoted overall uncertainty.

Single-top events are generated using ACERMC [47] for the $t$-channel and MC@NLO for the $W t$ - and $s-$ channels. The production of $W$ and $Z$ bosons in association with jets is simulated using the ALPGEN generator interfaced to HERWIG and JIMMY. Simulated $W+$ jets events are reweighted using the NLO PDF set CT10. Pairs of $W / Z$ bosons $(W W$, $W Z, Z Z)$ are produced using HERWIG.

All simulated samples are generated with multiple $p p$ interactions per bunch crossing (event pile-up). Up to 24 interactions per bunch crossing were observed during the data taking period. The number of interaction vertices in simulated samples is adjusted so that its distribution reproduces the one observed in data. The samples are then processed through the GEANT4 [48] simulation [49] of the ATLAS detector and the same reconstruction software used for data.

\subsection{Event selection}

Candidate events with the $t \bar{t}$ single-lepton signature are considered. These events are characterised by exactly one high $-p_{\mathrm{T}}$ isolated lepton (electron, muon or tau decaying to electron or muon), missing transverse momentum $E_{\mathrm{T}}^{\text {miss }}$ due to the neutrino from the leptonic $W$ decay, two jets originating from $b$-quarks and two jets originating from light quarks from the hadronic $W$ decay.

\footnotetext{
${ }^{1}$ ATLAS uses a right-handed coordinate system with its origin at the nominal interaction point (IP) in the centre of the detector and the $z$-axis along the beam pipe. The $x$-axis points from the IP to the centre of the LHC ring, and the $y$-axis points upward. Cylindrical coordinates $(r, \phi)$ are used in the transverse plane, $\phi$ being the azimuthal angle around the beam pipe. The pseudorapidity is defined in terms of the polar angle $\theta$ as $\eta=-\ln \tan (\theta / 2)$. Transverse momentum and energy are defined as $p_{\mathrm{T}}=p \sin \theta$ and $E_{\mathrm{T}}=E \sin \theta$, respectively.
} 
Events are required to pass the single-electron or single-muon trigger, with thresholds in transverse energy $\left(E_{T}\right)$ at $20 \mathrm{GeV}$ or $22 \mathrm{GeV}$ for electrons (depending on instantaneous luminosity conditions during the different data collection periods) and in transverse momentum $\left(p_{T}\right)$ at $18 \mathrm{GeV}$ for muons. Electron candidates are required to have $E_{\mathrm{T}}>25 \mathrm{GeV}$ and $\left|\eta_{\text {cluster }}\right|<2.47$, where $\eta_{\text {cluster }}$ is the pseudorapidity of the electromagnetic energy cluster in the calorimeter. Candidates in the transition region $1.37<\left|\eta_{\text {cluster }}\right|<1.52$ between calorimeter sections are excluded. Muon candidates are required to have $p_{\mathrm{T}}>20 \mathrm{GeV}$ and $|\eta|<2.5$. Electrons and muons are required to be isolated to reduce the backgrounds from hadrons mimicking lepton signatures and heavy-flavour decays inside jets. For electrons, stringent cuts both on the shape of the calorimetric energy deposits and on the tracks used to compute the isolation, in order to reject the tracks related to photon conversions, are applied. Cuts that depend on $\eta$ and $E_{T}$ leading to a $90 \%$ efficiency are used in a cone of $\Delta R=0.2$ for the energy isolation and in a cone of $\Delta R=0.3$ for the track isolation around the electron candidate. For muons, the sum of track transverse momenta in a cone of $\Delta R=0.3$ around the muon is required to be less than $2.5 \mathrm{GeV}$, while the total energy deposited in a cone of $\Delta R=0.2$ around the muon is required to be less than $4 \mathrm{GeV}$.

Jets are reconstructed from topologically connected calorimetric energy clusters using the anti- $k_{t}$ algorithm [50] with a radius parameter $R=0.4$. They are first calibrated to the electromagnetic energy scale and then corrected to the hadronic energy scale using energy - and $\eta$-dependent correction factors obtained from simulation and control data analyses [51]. The compatibility of the jets with the primary vertex (defined as the vertex with the highest sum of the square of the transverse momenta of the tracks associated to it) is determined using the tracks associated with the jet (jet vertex fraction). Jets originating from the hadronisation of $b$-quarks are identified by combining the information from three $b$-tagging algorithms, based on the topology of $b$ - and $c$-hadron weak decays inside jets [52] and on the transverse and longitudinal impact parameter significance of each track within the jet [53]. These three tagging algorithms are combined into a single discriminating variable used to make the tagging decision. The operating point chosen corresponds to a $70 \%$ tagging efficiency for $b$-quarks. The rejection rate is about 150 for light-quark jets, 5 for charm jets and 14 for hadronically decaying $\tau$ leptons. All these numbers are evaluated in simulated $t \bar{t}$ events.

The missing transverse momentum is reconstructed from clusters of energy deposits in the calorimeters calibrated at the electromagnetic scale and corrected according to the energy scale of the associated physics object. Contributions from muons are included using their momentum measured by the inner tracking and muon spectrometer systems.

Jets within $\Delta R \equiv \sqrt{(\Delta \eta)^{2}+(\Delta \phi)^{2}}=0.2$ of an electron candidate are removed to avoid double counting electrons as jets. Subsequently, electrons and muons within $\Delta R=$ 0.4 of a jet axis and with $p_{\mathrm{T}}>20 \mathrm{GeV}$ are removed in order to reduce the contamination caused by leptons from hadron decays.

In the muon channel, events are required to satisfy $E_{\mathrm{T}}^{\mathrm{miss}}>20 \mathrm{GeV}$ and $E_{\mathrm{T}}^{\mathrm{miss}}+$ $m_{\mathrm{T}}(W)>60 \mathrm{GeV}$ in order to suppress the multi-jets background. ${ }^{2}$ In the electron channel,

\footnotetext{
${ }^{2}$ In events with a leptonic decay of a genuine $W$ boson, $m_{\mathrm{T}}(W)$ is the $W$ boson transverse mass, defined as $\sqrt{2 p_{\mathrm{T}}^{\ell} p_{\mathrm{T}}^{\nu}\left(1-\cos \left(\phi^{\ell}-\phi^{\nu}\right)\right)}$, where the measured $E_{\mathrm{T}}^{\mathrm{miss}}$ vector provides the neutrino information.
} 
the multi-jets contamination is larger, and more stringent cuts of $E_{\mathrm{T}}^{\mathrm{miss}}>30 \mathrm{GeV}$ and $m_{\mathrm{T}}(W)>30 \mathrm{GeV}$ are applied.

Finally, events are required to have at least four jets with $p_{\mathrm{T}}>25 \mathrm{GeV}$ and $|\eta|<2.5$. These requirements define the 'pretag' selection. For the 'tag' selection, at least one of these jets is required to be $b$-tagged.

\subsection{Background estimation}

The main backgrounds affecting the measurement come from $W$ bosons produced in association with jets $(W+$ jets), single-top, $Z+$ jets, production of $W / Z$ bosons pairs and multi-jet events with background leptons. ${ }^{3}$ The $W+$ jets and multi-jets contributions are evaluated using a data-driven approach. Single-top, $Z+$ jets and diboson production are evaluated using simulated samples normalised to the approximate NNLO cross section for single-top events, NNLO cross section for inclusive $Z$ events, and NLO cross section for diboson events, respectively.

For reconstructed $t \bar{t}$ candidate events, the dominant $W+$ jets background is asymmetric in $\Delta|y|$ and therefore a data-driven technique is used to estimate its normalisation. The approach used is based on the fact that the production rate of $W^{+}+$jets is larger than that of $W^{-}+$jets. Since, to a good approximation, processes other than $W+$ jets give equal numbers of positively and negatively charged leptons, the formula

$$
N_{W^{+}}+N_{W^{-}}=\left(\frac{r_{\mathrm{MC}}+1}{r_{\mathrm{MC}}-1}\right)\left(D^{+}-D^{-}\right),
$$

is used to estimate the total number of $W$ events in the selected sample, after the numbers of single-top, diboson and $Z+$ jets events are evaluated in simulated samples and subtracted. Here, $N_{W^{ \pm}}$is the estimated number of $W^{ \pm}+$jets events, $D^{+}\left(D^{-}\right)$is the total number of events in data passing the pretag selection described in section 2.2 with positively (negatively) charged leptons, and $r_{\mathrm{MC}}=N\left(p p \rightarrow W^{+}+X\right) / N\left(p p \rightarrow W^{-}+X\right)$ is evaluated from simulation, using the ALPGEN generator with the same event selection. Further details of the method can be found in ref. [30].

The $W$ charge asymmetry depends also on the $W+$ jets flavour composition, i.e. on the mixture of $W b b+$ jets, $W c c+$ jets, $W c+$ jets and $W+$ light-jets processes in ALPGEN simulated samples. Since this composition cannot be predicted with sufficient precision, data-driven corrections are derived. The relative fractions are estimated in data, after subtracting all non- $W$ contributions, including $t \bar{t}$, applying the tag selection but requiring the presence of exactly two jets in the final state, in order to have a control region dominated by $W+$ jets events. The overall number of $W+$ jets events is determined simultaneously with the heavy-flavour composition in this region. The heavy-flavour fractions in the simulated $W+$ jets samples are then rescaled to the measured fractions. For the electron channel, the scale factors obtained are: $1.4 \pm 0.4$ for $W b b+$ jets and $W c c+$ jets, $0.7 \pm 0.4$ for $W c+$ jets and $1.00 \pm 0.10$ for $W+$ light-jets components. For the muon channel, they are: $1.2 \pm 0.4$ for $W b b+$ jets and $W c c+$ jets, $1.0 \pm 0.4$ for $W c+$ jets and $0.97 \pm 0.09$ for $W+$ light-jets

\footnotetext{
${ }^{3}$ The term 'background (bkgd) leptons' in this paper refers to hadrons mimicking lepton signatures and to leptons arising from heavy-hadron decays or photon conversions.
} 


\begin{tabular}{|c|c|c|c|c|}
\hline Channel & $\mu+$ jets pretag & $\mu+$ jets tag & $e+$ jets pretag & $e+$ jets tag \\
\hline$t \bar{t}$ & $34900 \pm 2200$ & $30100 \pm 1900$ & $21400 \pm 1300$ & $18500 \pm 1100$ \\
\hline$W+$ jets & $28200 \pm 3100$ & $4800 \pm 900$ & $13200 \pm 1600$ & $2300 \pm 900$ \\
\hline Multi-jets & $5500 \pm 1100$ & $1800 \pm 400$ & $3800 \pm 1900$ & $800 \pm$ \\
\hline Single top & $2460 \pm 120$ & $1970 \pm$ & $1530 \pm$ & $1220 \pm$ \\
\hline$Z+$ jets & $3000 \pm 1900$ & $480 \pm 230$ & $3000 \pm 1400$ & $460 \pm 220$ \\
\hline Diboson & $380 \pm 180$ & $80 \pm$ & $230 \pm 110$ & $47 \pm$ \\
\hline Total background & $40000 \pm 4000$ & $9200 \pm 1000$ & $21700 \pm 2900$ & $4800 \pm 1000$ \\
\hline Signal + background & $74000 \pm 4000$ & $39300 \pm 2100$ & $43100 \pm 3100$ & $23300 \pm 1600$ \\
\hline Observed & 70845 & 37568 & 40972 & 21929 \\
\hline
\end{tabular}

Table 1. Numbers of expected events for the $t \bar{t}$ signal and the various background processes and observed events in data for the pretag and tag samples. The uncertainties include statistical and systematic components.

components. The uncertainties include both the statistical and the systematic components. The sources of systematic uncertainty considered are those described in section 3.3.

With the determined flavour fractions, the $W+$ jets normalisation for pretag-selected events using eq. (2.1) is computed and then extrapolated to the tag-selected events using the tagging fractions (i.e. the fraction of events with at least one $b$-jet) computed in simulated samples. The scale factors that are applied to the tag-selected $W+$ jets events are $0.83 \pm 0.31$ and $0.94 \pm 0.17$ in the electron and muon channel respectively. The uncertainties include both the statistical and the systematic components, including a particular systematic uncertainty that accounts for differences in the flavour composition between the signal region and the region where the flavour fractions are extracted. It is derived from studies of ALPGEN parameter variations (factorisation and renormalisation scales, angular matching parameters and jet $p_{\mathrm{T}}$ generation thresholds) and it amounts to $15 \%$ for the $W b b / W c c / W c+$ jets components and $5 \%$ for the $W+$ light-jets component.

The 'Matrix Method' is used to evaluate the multi-jets background with background leptons. The method relies on defining 'loose' and 'tight' lepton samples [54] and measuring the 'tight' selection efficiencies for real $\left(\epsilon_{\text {real }}\right)$ and background $\left(\epsilon_{\mathrm{bkgd}}\right)$ 'loose' leptons. The 'loose' selection requires less stringent identification and isolation requirements than the ones described in section 2.2, referred here as 'tight' selection. The fraction $\epsilon_{\text {real }}$ is measured using data control samples of $Z$ boson decays to two leptons. The fraction $\epsilon_{\mathrm{bkgd}}$ is measured in control regions where the contribution of background leptons is dominant.

The expected and observed yields are listed in table 1 . The number of events in the electron channel is significantly lower than in the muon channel due to the higher lepton $p_{\mathrm{T}}$ threshold, tighter isolation and the more stringent missing transverse momentum requirements. The number of events observed in data and the total predicted yield are compatible within uncertainty. 


\section{The $t \bar{t}$ production charge asymmetry measurement}

After the reconstruction of the $t \bar{t}$-system (section 3.1) and the estimation of the background, the $\Delta|y|$ spectra (section 3.2) are unfolded to obtain inclusive and differential partonlevel charge asymmetry measurements (as a function of $m_{t \bar{t}}, p_{\mathrm{T}, t \bar{t}}$ and $\left|y_{t \bar{t}}\right|$ ), as defined in eq. (1.1).

In addition, an inclusive measurement and a differential measurement as a function of $m_{t \bar{t}}$ are performed for events where the $z$-component of the $t \bar{t}$-system velocity is large, $\beta_{z, t \bar{t}}>0.6$. Most BSM models introduced to explain the excesses in the CDF and D0 measurements postulate the presence of new particles that can alter the SM prediction for $A_{\mathrm{C}}$. Requiring $\beta_{z, t \bar{t}}>0.6$ defines a region of phase-space where the effects of these new particles on the asymmetry are enhanced [32].

\subsection{Reconstruction of the $t \bar{t}$-system}

A kinematic fit is used to determine the likelihood for candidate events to be $t \bar{t}$ events as well as to determine the four-vector of the top quark and antiquark to compute $\Delta|y|$. The charge of the lepton is used to determine whether the reconstructed object is a top quark or antiquark. A detailed description of the method and its assumptions can be found in ref. [30]. In simulation studies using $t \bar{t}$ events, the fraction of events reconstructed with the correct $\Delta|y|$ sign was evaluated to be $75 \%$.

For the differential measurements a cut on the likelihood is applied to reject badly reconstructed events, reducing the migrations across the bins. The reconstructed $\Delta|y|$ distribution is shown in figure 1 along with the distributions of $m_{t \bar{t}}, p_{\mathrm{T}, t \bar{t}},\left|y_{t \bar{t}}\right|$ and $\beta_{z, t \bar{t}}$.

\subsection{Unfolding procedure}

The reconstructed $\Delta|y|$ distributions are distorted by acceptance and detector resolution effects. We use the Fully Bayesian Unfolding (FBU) [55] technique to estimate the partonlevel distributions from the measured spectra. This method relies on applying Bayes' theorem to the unfolding problem, which can be formulated in the following terms.

Given an observed data spectrum $\mathbf{D} \in \mathbb{N}^{N_{\mathrm{r}}}$ and a migration matrix $\mathcal{M} \in \mathbb{R}^{N_{\mathrm{r}}} \times \mathbb{R}^{N_{\mathrm{t}}}$ ( $N_{\mathrm{r}}$ and $N_{\mathrm{t}}$ are the number of bins in the measured and true spectra respectively) that takes into account the distortion effects mentioned above, the posterior probability density of the true spectrum $\mathbf{T} \in \mathbb{R}^{N_{\mathrm{t}}}$ follows the probability density

$$
p(\mathbf{T} \mid \mathbf{D}, \mathcal{M}) \propto \mathcal{L}(\mathbf{D} \mid \mathbf{T}, \mathcal{M}) \cdot \pi(\mathbf{T})
$$

where $\mathcal{L}(\mathbf{D} \mid \mathbf{T}, \mathcal{M})$ is the conditional likelihood for the data $\mathbf{D}$ assuming the true $\mathbf{T}$ and the migration matrix $\mathcal{M}$, and $\pi$ is the prior probability density for the true $\mathbf{T}$.

Assuming that the data follows a Poisson distribution, the likelihood $\mathcal{L}(\mathbf{D} \mid \mathbf{T}, \mathcal{M})$ can be computed starting from the migration matrix $\mathcal{M}$, whose elements $\mathcal{M}_{t r}$ represent the probability and the efficiency of an event produced in the true bin $t$ to be reconstructed in any bin $r$. The background in each bin is taken into account when computing $\mathcal{L}(\mathbf{D} \mid \mathbf{T}, \mathcal{M})$. While the above quantities can be estimated from simulated samples of signal events, the prior probability density $\pi(\mathbf{T})$ must be chosen according to what is known about $\mathbf{T}$ before 

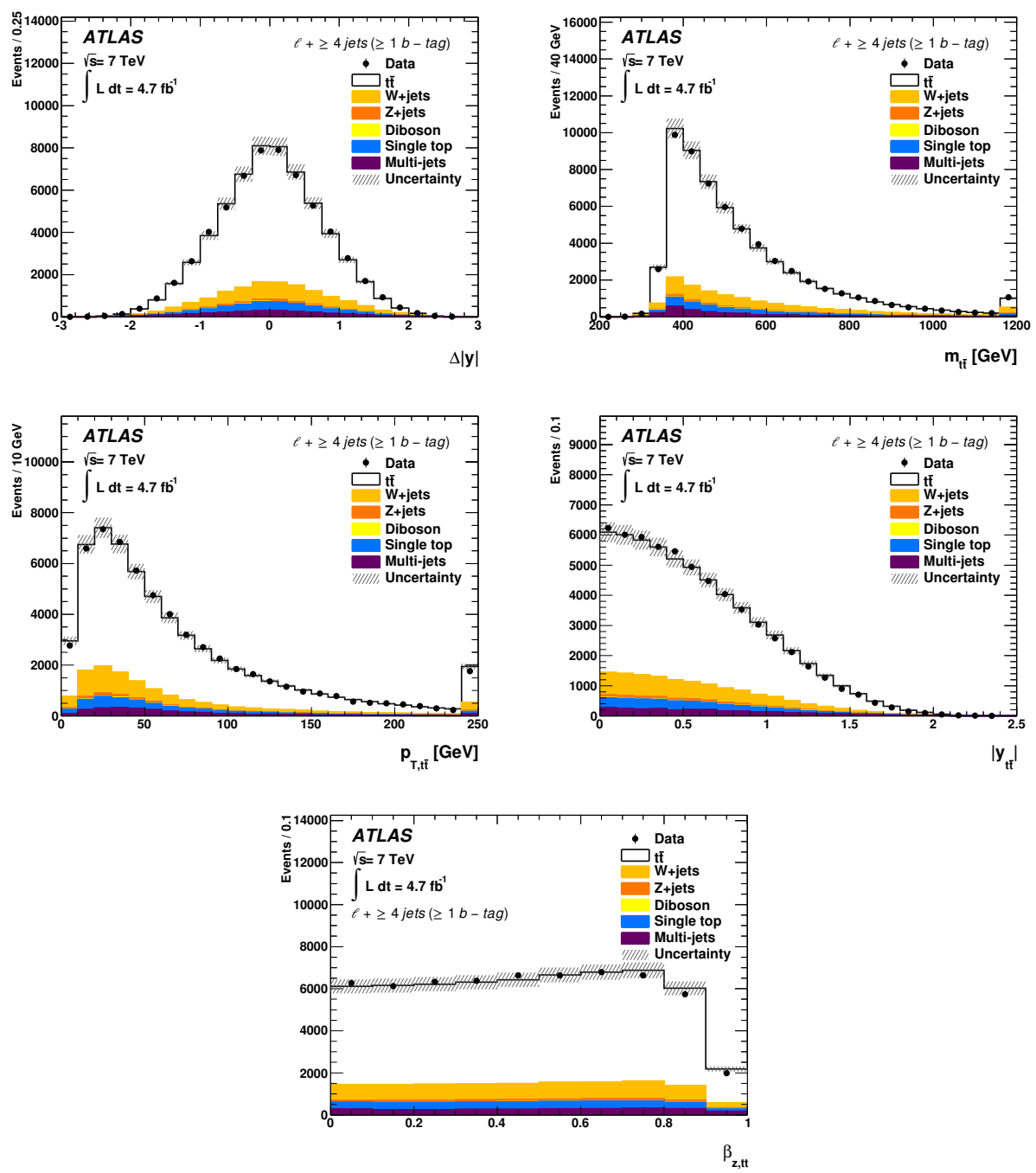

Figure 1. Reconstructed $\Delta|y|$ (top left), invariant mass $m_{t \bar{t}}$ (top right), transverse momentum $p_{\mathrm{T}, t \bar{t}}$ (centre left), rapidity $\left|y_{t \bar{t}}\right|$ (centre right) and velocity $\beta_{z, t \bar{t}}$ (bottom) distributions for the electron and muon channels combined after requiring at least one $b$-tagged jet. Data (dots) and SM expectations (solid lines) are shown. The uncertainty on the total prediction includes both the statistical and the systematic components. The overflow is included in the last bin.

the measurement. In this context, the choice of the prior can be interpreted as the choice of a regularisation in other unfolding techniques (see ref. [56] for instance). After choosing a prior, the posterior probability density $p(\mathbf{T} \mid \mathbf{D}, \mathcal{M})$ is computed by generating uniformly distributed points in the $N_{\mathrm{t}}$-dimensional space, and evaluating for each of them $\mathcal{L}(\mathbf{D} \mid \mathbf{T}, \mathcal{M})$ and $\pi(\mathbf{T})$. A weight given by $\mathcal{L}(\mathbf{D} \mid \mathbf{T}, \mathcal{M}) \cdot \pi(\mathbf{T})$ is then assigned to each point, allowing the posterior probability density of the unfolded spectrum to be determined, for each $\Delta|y|$ bin and for $A_{\mathrm{C}}$.

The FBU method has two main advantages. Firstly, it gives a precise physical meaning to the regularisation procedure through the choice of a prior built with well-motivated physical quantities. Secondly, systematic uncertainties are accounted for consistently with 
the Bayesian statistical approach, by reporting credible intervals built by integrating the posterior distribution over the nuisance parameters.

The choice of the prior is arbitrary. With a flat prior, the FBU method has been checked to be equivalent to unregularised matrix inversion. Non-uniform priors favour spectra that have some well-defined features. By assuming that some spectra are more likely than others, information is added to the measurement, reducing the uncertainty but potentially biasing its outcome.

Two different priors are used in the following: a flat prior and a curvature prior. The curvature prior is defined starting from the definition of the curvature $C(\mathbf{T})$ being the sum of the squares of the second derivatives of the $\Delta|y|$ distribution $\mathbf{T}$ with $N_{\mathrm{t}}$ bins:

$$
C(\mathbf{T})=\sum_{i=2}^{N_{\mathrm{t}}-1}\left(\Delta_{i+1, i}-\Delta_{i, i-1}\right)^{2}
$$

where $\Delta_{a, b}=T_{a}-T_{b}$. The curvature prior is then defined as follows:

$$
\pi(\mathbf{T}) \propto \begin{cases}\mathrm{e}^{\alpha \mathrm{S}(\mathbf{T})} & \text { in the integration space, } \forall t \in\left[1, N_{\mathrm{t}}\right] \\ 0 & \text { otherwise }\end{cases}
$$

where $\alpha$ is the regularisation parameter and $S(\mathbf{T}) \equiv\left|C(\mathbf{T})-C\left(\mathbf{T}^{*}\right)\right|$ is a regularisation function, defined, for each generated point, as the difference between the curvature $C(\mathbf{T})$ of the true $\Delta|y|$ spectrum $\mathbf{T}$ and that of the estimated spectrum $\mathbf{T}^{*}$.

The flat prior is used for the differential measurements of $A_{\mathrm{C}}$ as a function of $m_{t \bar{t}}$ and of $\left|y_{t \bar{t}}\right|$. The curvature prior defined in eq. (3.2) is used for the inclusive measurement and for the differential measurement as a function of $p_{\mathrm{T}, t \bar{t}}$, because it reduces the uncertainty on these measurements. The regularisation strength $\alpha=10^{-8}$ is chosen based on the numerical value of the curvature of the true spectrum. It has been checked, by varying $\alpha$ by one order of magnitude included the $\alpha=0$ unregularised case, that this particular choice of $\alpha$ does not cause any significant bias in either the unfolded distributions or in the computed asymmetries. The consistency of the FBU method with the iterative scheme [56] has been checked as well.

Four bins are used for the $\Delta|y|$ distribution both for the inclusive and the differential measurements. The $\Delta|y|$ bin ranges are the same in both measurements. The bin ranges for the differential variables are chosen to have approximately the same number of entries in each bin. The $A_{\mathrm{C}}$ posterior probability density is built from the asymmetry in each generated point of the integration space. The value of $A_{\mathrm{C}}$ and its statistical uncertainty are the mean and the RMS of the posterior probability density distribution respectively.

\subsection{Systematic uncertainties}

Several sources of systematic uncertainty are taken into account.

A possible small mis-modelling of the lepton momentum scale and resolution in simulation is corrected by scale factors derived from the comparison of $Z \rightarrow \ell \ell, J / \psi \rightarrow \ell \ell$ and $W \rightarrow e \nu$ events in data and simulation. The uncertainty on the scale factors ranges from $1 \%$ to $1.5 \%$ depending on the $p_{\mathrm{T}}$ and $\eta$ of the leptons. 
The jet energy scale is derived using information from test-beam data, collision data and simulation. Its uncertainty is between $1 \%$ and $2.5 \%$ in the central region of the detector, depending on jet $p_{\mathrm{T}}$ and $\eta$ [51]. This value includes uncertainties due to the flavour composition of the sample, mis-measurements due to the effect of nearby jets, influence of pile-up, and a $p_{\mathrm{T}}$-dependent uncertainty for jets arising from the fragmentation of $b$-quarks. The jet energy resolution and reconstruction efficiencies are measured in data using techniques described in refs. [51, 57].

The uncertainties on the lepton and jets are propagated to the missing transverse momentum calculation.

The $b$-tagging efficiencies and light jets mis-tag rates are measured in data. Jet $p_{\mathrm{T}^{-}}$ dependent scale factors are applied to simulation to match the efficiencies observed in data. The typical uncertainty on the $b$-tagging scale factors ranges from $6 \%$ to $20 \%$ (depending on jet $p_{\mathrm{T}}$ and $\eta$ ) for $b$-jets, from $12 \%$ to $22 \%$ for $c$-jets and is about $16 \%$ for light-jets [53]. The impact of this uncertainty is negligible.

The systematic uncertainty in the modelling of the signal process is assessed by varying the simulation parameters and by using a different Monte Carlo generator (POWHEG [58, 59]). The sources of systematic uncertainty considered are the choice and the functional form of factorisation scale and the choice of parton shower model (PYTHIA or HERWIG). The impact of the choice of PDFs is evaluated following the procedure described in ref. [46]. All these uncertainties have a negligible impact on the asymmetry.

The limited size of the MC simulation samples gives rise to a systematic uncertainty in the response matrix. This is estimated by independently varying the bin content of the response matrix according to Poisson distributions.

Several other sources of systematic uncertainties are considered, namely the uncertainties on: the luminosity determination (1.8\%) [34], the lepton and trigger reconstruction and identification scale factors, the lepton charge mis-identification, the jet vertex fraction scale factor, the missing transverse momentum scale and resolution and the $Z+$ jets and multi-jets background normalisations. All of these lead to uncertainties on the asymmetry measurements below 0.001 and are therefore negligible.

Systematic uncertainties related to the different choice of PDFs and to the shape of the $W+$ jets distributions are also considered. The former is evaluated as explained above. The latter is estimated in simulated events generated with the same variations of the ALPGEN parameters as described above for the modelling of the signal process.

For each of the systematic uncertainties (except for those related to the modelling of the $t \bar{t}$ signal and for the $W+$ jets shape) the $W+$ jets normalisation and the heavy-flavour composition are recomputed as described in section 2.3 to take into account the correlation with the various sources of systematic uncertainty considered.

For the systematic uncertainties affecting the background, the posterior probability density with a modified background prediction is computed. For those affecting the signal, the posterior probability density with the modified efficiency and response matrix is evaluated.

Systematic uncertainties are taken into account with a marginalisation procedure. After computing the posterior probability density corresponding to each systematic variation, 


\begin{tabular}{|l|c|c|}
\hline$A_{\mathrm{C}}$ & Data & Theory \\
\hline Unfolded & $0.006 \pm 0.010$ & $0.0123 \pm 0.0005$ \\
Unfolded with $m_{t \bar{t}}>600 \mathrm{GeV}$ & $0.018 \pm 0.022$ & $0.0175_{-0.0004}^{+0.0005}$ \\
Unfolded with $\beta_{z, t \bar{t}}>0.6$ & $0.011 \pm 0.018$ & $0.020_{-0.007}^{+0.006}$ \\
\hline
\end{tabular}

Table 2. Measured inclusive charge asymmetry, $A_{\mathrm{C}}$, values for the electron and muon channels combined after unfolding without and with the $\beta_{z, t \bar{t}}>0.6$ cut explained in the text. The $A_{\mathrm{C}}$ measurement with a cut on the $t \bar{t}$ invariant mass $m_{t \bar{t}}>600 \mathrm{GeV}$ is also shown. SM predictions, as described in the text, are also reported. The quoted uncertainties include statistical and systematic components after the marginalisation.

the likelihood used in the unfolding is marginalised by integrating out its dependence on the nuisance parameters. It is assumed that the priors for all nuisance parameters are Gaussian and that there is no correlation between them. A marginalisation is then performed by transforming the integral over the nuisance parameter into a discrete sum of the posterior probability densities evaluated at three values of the nuisance parameter: the central one and the $1 \sigma$ variations. The resulting posterior probability density is finally used to extract the systematic uncertainty on the measurements.

\section{Results}

\subsection{Inclusive and differential measurements}

The $t \bar{t}$ production charge asymmetry is measured to be $A_{\mathrm{C}}=0.006 \pm 0.010$ compatible with the SM prediction $A_{\mathrm{C}}=0.0123 \pm 0.0005$ [21]. These values are shown in table 2 together with the measurement and prediction for $m_{t \bar{t}}>600 \mathrm{GeV}$. The total systematic uncertainty is computed with the marginalisation procedure described in section 3.3. The uncertainties quoted for all the results in this section include statistical and systematic components. In order to estimate the impact of each source of systematic uncertainty, the marginalisation procedure is repeated removing one such source at a time from the global marginalisation. For each of the systematic uncertainties considered in this analysis and for all the measurements, the impact on the $A_{\mathrm{C}}$ value and its uncertainty is less than $10 \%$ of the statistical uncertainty, and thus negligible.

As a cross-check, the systematic uncertainties affecting $A_{\mathrm{C}}$ are computed one by one before the marginalisation procedure described above. For each source, the systematic uncertainty represents the variation of the mean of posterior probability densities corresponding to a $1 \sigma$ variation of the nuisance parameter. The statistical uncertainty still dominates the variations in $A_{\mathrm{C}}$ even before the marginalisation procedure. Table 3 summarises the result of this 'cross-check' procedure for the inclusive charge asymmetry measurement (left column) and for the measurement with the $m_{t \bar{t}}>600 \mathrm{GeV}$ requirement after unfolding (central column). Figure 2 shows the charge asymmetry as a function of $m_{t \bar{t}}, p_{\mathrm{T}, t \bar{t}}$ and $\left|y_{t \bar{t}}\right|$ compared with the theoretical SM predictions described in ref. [21] and provided by its authors for the chosen bins. In addition, predictions for two assumed mass values (300 $\mathrm{GeV}[14]$ and $7000 \mathrm{GeV}$ ), for a heavy axigluon exchanged in the $s$-channel, are also shown. 


\begin{tabular}{|l|c|c|c|}
\hline Source of systematic uncertainty & \multicolumn{3}{|c|}{$\delta A_{\mathrm{C}}$} \\
\hline Lepton reconstruction/identification & Inclusive & $m_{t \bar{t}}>600 \mathrm{GeV}$ & $\beta_{z, t \bar{t}}>0.6$ \\
Lepton energy scale and resolution & $<0.001$ & 0.001 & $<0.001$ \\
Jet energy scale and resolution & 0.003 & 0.003 & 0.003 \\
Missing transverse momentum and pile-up modelling & 0.003 & 0.003 & 0.005 \\
Multi-jets background normalisation & $<0.001$ & 0.002 & 0.004 \\
$b$-tagging/mis-tag efficiency & $<0.001$ & 0.001 & 0.001 \\
Signal modelling & $<0.001$ & $<0.001$ & $<0.001$ \\
Parton shower/hadronisation & $<0.001$ & $<0.001$ & $<0.001$ \\
Monte Carlo statistics & 0.002 & $<0.001$ & $<0.001$ \\
PDF & 0.001 & $<0.001$ & $<0.001$ \\
$W+$ jets normalisation and shape & 0.002 & $<0.001$ & $<0.001$ \\
\hline Statistical uncertainty & 0.010 & 0.021 & 0.017 \\
\hline
\end{tabular}

Table 3. Systematic uncertainties for the inclusive asymmetry, $A_{\mathrm{C}}$ (second column), the asymmetry for $m_{t \bar{t}}>600 \mathrm{GeV}$ (third column) and the inclusive asymmetry, $A_{\mathrm{C}}$, for $\beta_{z, t \bar{t}}>0.6$ (fourth column). For variations resulting in asymmetric uncertainties, the average absolute deviation from the nominal value is reported. The values reported for each systematic uncertainty are the variation of the mean of posteriors computed considering $1 \sigma$ variations.

The masses are chosen as benchmarks, taking into account the fact that they would not be visible as resonances in the $m_{t \bar{t}}$ spectrum. The parameters of the model are tuned to give a forward-backward asymmetry compatible with the Tevatron results. The differential distributions and respective asymmetries do not show any significant deviation from the SM prediction. The resulting charge asymmetry $A_{\mathrm{C}}$ is shown in table 4 for the differential measurements as a function of $m_{t \bar{t}}, p_{\mathrm{T}, t \bar{t}}$ and $\left|y_{t \bar{t}}\right|$. The systematic uncertainties, computed before the marginalisation procedure as described above in the cross-check procedure, are listed in table 5 for each of the differential measurements. The correlation matrices for the statistical uncertainties are shown in table 6 for the measurement as a function of $m_{t \bar{t}}, p_{\mathrm{T}, t \bar{t}}$ and $\left|y_{t \bar{t}}\right|$ respectively.

\subsection{Measurements for $\beta_{z, t \bar{t}}>0.6$}

An additional requirement on the $z$-component of the $t \bar{t}$-system velocity $\beta_{z, t \bar{t}}>0.6$ is applied, as explained in section 1, for the inclusive and the differential $\Delta|y|$ distribution as a function of $m_{t \bar{t}}$. It has been verified that resolution effects on the reconstructed $\beta_{z, t \bar{t}}$ did not introduce any bias in the measurement. Hence an unfolding of the $\beta_{z, t \bar{t}}$ distribution was found to be unnecessary. The inclusive asymmetry after this requirement is $A_{\mathrm{C}}=0.011 \pm 0.018$, as reported in the last row of table 2 , to be compared with the $\mathrm{SM}$ prediction $A_{\mathrm{C}}^{\mathrm{SM}}=0.020_{-0.007}^{+0.006}[21]$. Table 3 (right column) shows the list of systematic uncertainties affecting the measurement before the marginalisation procedure.

Figure 2 (bottom right plot) shows the differential $A_{\mathrm{C}}$ measurement as a function of $m_{t \bar{t}}$, while table 7 shows the value of $A_{\mathrm{C}}$ for the different bins, table 8 lists the systematic uncertainties affecting the measurement before the marginalisation and table 9 shows 

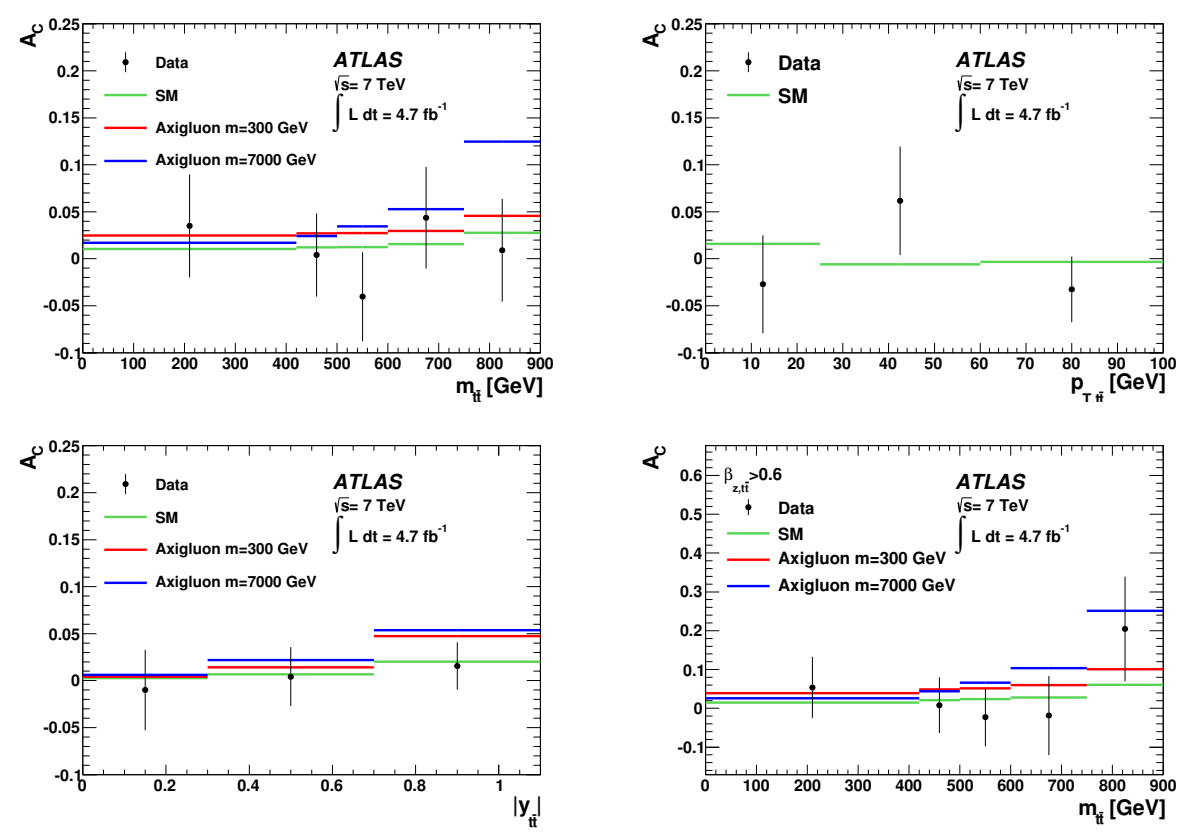

Figure 2. Distributions of $A_{\mathrm{C}}$ as a function of $m_{t \bar{t}}$ (top left), $p_{\mathrm{T}, t \bar{t}}$ (top right) and $\left|y_{t \bar{t}}\right|$ (bottom left) after unfolding, for the electron and muon channels combined. The $A_{\mathrm{C}}$ distribution as a function of $m_{t \bar{t}}$, after the $\beta_{z, t \bar{t}}>0.6$ requirement, is also shown (bottom right). The $A_{\mathrm{C}}$ values after the unfolding (points) are compared with the SM predictions (green lines) and the predictions for a colour-octet axigluon with a mass of $300 \mathrm{GeV}$ (red lines) and $7000 \mathrm{GeV}$ (blue lines) respectively, as described in the text. The thickness of the lines represents the factorisation and renormalisation scale uncertainties on the corresponding theoretical predictions. The values plotted are the average $A_{\mathrm{C}}$ in each bin. The error bars include both the statistical and the systematic uncertainties on $A_{\mathrm{C}}$ values. The bins are the same as the ones reported in tables 4 and 7 respectively.

the correlation coefficients among the different bins. These measurements do not deviate significantly from the SM expectations either.

\subsection{Interpretation}

Figure 3 shows the inclusive $A_{\mathrm{C}}$ measurements with and without the additional requirement on the invariant mass of the $t \bar{t}$-system $m_{t \bar{t}}>600 \mathrm{GeV}$ described in section 4.1. In the left plot, the $A_{\mathrm{C}}$ measurement without the $m_{t \bar{t}}>600 \mathrm{GeV}$ requirement is compared with the corresponding measurement from CMS [29] (horizontal lines) and with the $t \bar{t}$ forward-backward asymmetry $A_{\mathrm{FB}}$ measurements made at the Tevatron by CDF, $A_{\mathrm{FB}}=0.164 \pm 0.045$ [24], and D0, $A_{\mathrm{FB}}=0.196 \pm 0.065$ [26] (vertical lines). In the right plot, the $A_{\mathrm{C}}$ measurement with the requirement of $m_{t \bar{t}}>600 \mathrm{GeV}$, is compared with the $A_{\mathrm{FB}}$ measurement, with the requirement of $m_{t \bar{t}}>450 \mathrm{GeV}$, performed by the CDF experiment at the Tevatron [24].

Predictions given by several new physics models introduced to explain the larger than expected $A_{\mathrm{FB}}$ values measured at the Tevatron are also displayed. Details of these models can be found in refs. $[8,30,60]$. For each model, the predictions for $A_{\mathrm{FB}}$ and $A_{\mathrm{C}}$ are derived 


\begin{tabular}{|l|c|c|c|c|c|}
\hline & \multicolumn{5}{|c|}{$m_{t \bar{t}}[\mathrm{GeV}]$} \\
\hline$A_{\mathrm{C}}$ & $0-420$ & $420-500$ & $500-600$ & $600-750$ & $>750$ \\
\hline Unfolded & $0.036 \pm 0.055$ & $0.003 \pm 0.044$ & $-0.039 \pm 0.047$ & $0.044 \pm 0.054$ & $0.011 \pm 0.054$ \\
Theory & $0.0103_{-0.0004}^{+0.0003}$ & $0.0123_{-0.0003}^{+0.0006}$ & $0.0125 \pm 0.0002$ & $0.0156_{-0.0009}^{+0.0007}$ & $0.0276_{-0.0008}^{+0.0004}$ \\
\hline
\end{tabular}

\begin{tabular}{|l|c|c|c|}
\hline & \multicolumn{3}{|c|}{$p_{\mathrm{T}, t \bar{t}}[\mathrm{GeV}]$} \\
\hline$A_{\mathrm{C}}$ & $0-25$ & $25-60$ & $>60$ \\
\hline Unfolded & $-0.032 \pm 0.052$ & $0.067 \pm 0.057$ & $-0.034 \pm 0.034$ \\
Theory & $0.0160_{-0.0009}^{+0.0007}$ & $-0.0058_{-0.0004}^{+0.0004}$ & $-0.0032_{-0.0002}^{+0.0002}$ \\
\hline
\end{tabular}

\begin{tabular}{|l|c|c|c|}
\hline & \multicolumn{3}{|c|}{$\left|y_{t \bar{t}}\right|$} \\
\hline$A_{\mathrm{C}}$ & $0-0.3$ & $0.3-0.7$ & $>0.7$ \\
\hline Unfolded & $-0.010 \pm 0.043$ & $0.006 \pm 0.031$ & $0.015 \pm 0.025$ \\
Theory & $0.0026_{-0.0001}^{+0.0008}$ & $0.0066_{-0.0003}^{+0.0001}$ & $0.0202_{-0.0007}^{+0.0006}$ \\
\hline
\end{tabular}

Table 4. Measured charge asymmetry, $A_{\mathrm{C}}$, values for the electron and muon channels combined after unfolding as a function of the $t \bar{t}$ invariant mass, $m_{t \bar{t}}$ (top), the $t \bar{t}$ transverse momentum, $p_{\mathrm{T}, t \bar{t}}$ (middle) and the $t \bar{t}$ rapidity, $\left|y_{t \bar{t}}\right|$ (bottom). SM predictions, as described in the text, are also reported. The quoted uncertainties include statistical and systematic components after the marginalisation.

using the PROTOS generator [61] with the constraints described in ref. [30]. The ranges of predicted values for $A_{\mathrm{FB}}$ and $A_{\mathrm{C}}$ for a given new physics model are also shown. The new physics contributions are computed using the tree-level SM amplitude plus the one(s) from the new particle(s), to account for the interference between the two contributions. Some of these new physics models seem to be disfavoured by the current measurements.

\section{Conclusion}

This paper has presented a measurement of the $t \bar{t}$ production charge asymmetry measurement in $t \bar{t}$-events with a single lepton (electron or muon), at least four jets, of which at least one is tagged as a $b$-jet, and large missing transverse momentum, using an integrated luminosity of $4.7 \mathrm{fb}^{-1}$ recorded by the ATLAS experiment in $p p$ collisions at a centre-of-mass energy of $\sqrt{s}=7 \mathrm{TeV}$ at the LHC. The inclusive $t \bar{t}$ production charge asymmetry $A_{\mathrm{C}}$ and its differential distributions, as a function of $m_{t \bar{t}}, p_{\mathrm{T}, t \bar{t}}$ and $\left|y_{t \bar{t}}\right|$, have been unfolded to parton-level. The measured inclusive $t \bar{t}$ production charge asymmetry is $A_{\mathrm{C}}=0.006 \pm 0.010$, to be compared with the SM prediction $A_{\mathrm{C}}^{\mathrm{SM}}=0.0123 \pm 0.0005$. All measurements presented are statistically limited and are found to be compatible with the SM prediction within the uncertainties.

\section{Acknowledgments}

We thank CERN for the very successful operation of the LHC, as well as the support staff from our institutions without whom ATLAS could not be operated efficiently. 


\begin{tabular}{|c|c|c|c|c|c|}
\hline & \multicolumn{5}{|c|}{$m_{t \bar{t}}[\mathrm{GeV}]$} \\
\hline Source of systematic uncertainty & $0-420$ & $420-500$ & $500-600$ & $600-750$ & $>750$ \\
\hline Lepton reconstruction/identification & $<0.005$ & $<0.005$ & $<0.005$ & $<0.005$ & $<0.005$ \\
\hline Lepton energy scale and resolution & 0.017 & 0.014 & 0.013 & 0.007 & $<0.005$ \\
\hline Jet energy scale and resolution & 0.014 & 0.007 & 0.035 & 0.032 & 0.017 \\
\hline Missing transverse momentum and pile-up modelling & 0.013 & 0.017 & 0.018 & 0.008 & 0.005 \\
\hline Multi-jets background normalisation & $<0.005$ & $<0.005$ & $<0.005$ & $<0.005$ & $<0.005$ \\
\hline$b$-tagging/mis-tag efficiency & $<0.005$ & $<0.005$ & $<0.005$ & $<0.005$ & $<0.005$ \\
\hline Signal modelling & $<0.005$ & $<0.005$ & $<0.005$ & $<0.005$ & $<0.005$ \\
\hline Parton shower/hadronisation & $<0.005$ & $<0.005$ & $<0.005$ & $<0.005$ & $<0.005$ \\
\hline Monte Carlo sample size & $<0.005$ & $<0.005$ & $<0.005$ & $<0.005$ & $<0.005$ \\
\hline $\mathrm{PDF}$ & $<0.005$ & $<0.005$ & $<0.005$ & $<0.005$ & $<0.005$ \\
\hline$W+$ jets normalisation and shape & $<0.005$ & $<0.005$ & $<0.005$ & $<0.005$ & $<0.005$ \\
\hline Statistical uncertainty & 0.054 & 0.042 & 0.046 & 0.052 & 0.054 \\
\hline
\end{tabular}

\begin{tabular}{|c|c|c|c|}
\hline & \multicolumn{3}{|c|}{$p_{\mathrm{T}, t \bar{t}}[\mathrm{GeV}]$} \\
\hline Source of systematic uncertainty & $0-25$ & $25-60$ & $>60$ \\
\hline Lepton reconstruction/identification & $<0.005$ & $<0.005$ & $<0.005$ \\
\hline Lepton energy scale and resolution & 0.011 & 0.013 & 0.006 \\
\hline Jet energy scale and resolution & 0.009 & 0.020 & 0.020 \\
\hline Missing transverse momentum and pile-up modelling & 0.017 & 0.010 & $<0.005$ \\
\hline Multi-jets background normalisation & $<0.005$ & $<0.005$ & $<0.005$ \\
\hline$b$-tagging/mis-tag efficiency & $<0.005$ & $<0.005$ & $<0.005$ \\
\hline Signal modelling & $<0.005$ & $<0.005$ & $<0.005$ \\
\hline Parton shower/hadronisation & $<0.005$ & $<0.005$ & $<0.005$ \\
\hline Monte Carlo sample size & $<0.005$ & $<0.005$ & $<0.005$ \\
\hline $\mathrm{PDF}$ & $<0.005$ & $<0.005$ & $<0.005$ \\
\hline$W+$ jets normalisation and shape & $<0.005$ & $<0.005$ & $<0.005$ \\
\hline \multirow[t]{2}{*}{ Statistical uncertainty } & 0.052 & 0.057 & 0.034 \\
\hline & \multicolumn{3}{|c|}{$\left|y_{t \bar{t}}\right|$} \\
\hline Source of systematic uncertainty & $0-0.3$ & $0.3-0.7$ & $>0.7$ \\
\hline Lepton reconstruction/identification & $<0.005$ & $<0.005$ & $<0.005$ \\
\hline Lepton energy scale and resolution & 0.022 & 0.014 & 0.008 \\
\hline Jet energy scale and resolution & 0.013 & 0.007 & $<0.005$ \\
\hline Missing transverse momentum and pile-up modelling & $<0.005$ & 0.006 & $<0.005$ \\
\hline Multi-jets background normalisation & $<0.005$ & $<0.005$ & $<0.005$ \\
\hline$b$-tagging/mis-tag efficiency & $<0.005$ & $<0.005$ & $<0.005$ \\
\hline Signal modelling & $<0.005$ & $<0.005$ & $<0.005$ \\
\hline Parton shower/hadronisation & $<0.005$ & $<0.005$ & $<0.005$ \\
\hline Monte Carlo sample size & $<0.005$ & $<0.005$ & $<0.005$ \\
\hline $\mathrm{PDF}$ & $<0.005$ & $<0.005$ & $<0.005$ \\
\hline$W+$ jets normalisation and shape & $<0.005$ & $<0.005$ & $<0.005$ \\
\hline Statistical uncertainty & 0.042 & 0.030 & 0.025 \\
\hline
\end{tabular}

Table 5. Systematic uncertainties for the charge asymmetry, $A_{\mathrm{C}}$, measurement for the electron and muon channels combined after unfolding as a function of the $t \bar{t}$ invariant mass, $m_{t \bar{t}}$ (top), the $t \bar{t}$ transverse momentum, $p_{\mathrm{T}, t \bar{t}}$ (middle) and the $t \bar{t}$ rapidity, $\left|y_{t \bar{t}}\right|$ (bottom). For variations resulting in asymmetric uncertainties, the average absolute deviation from the nominal value is reported. The values reported for each systematic uncertainty are the variation of the mean of posterior probability densities computed considering $1 \sigma$ variations. 


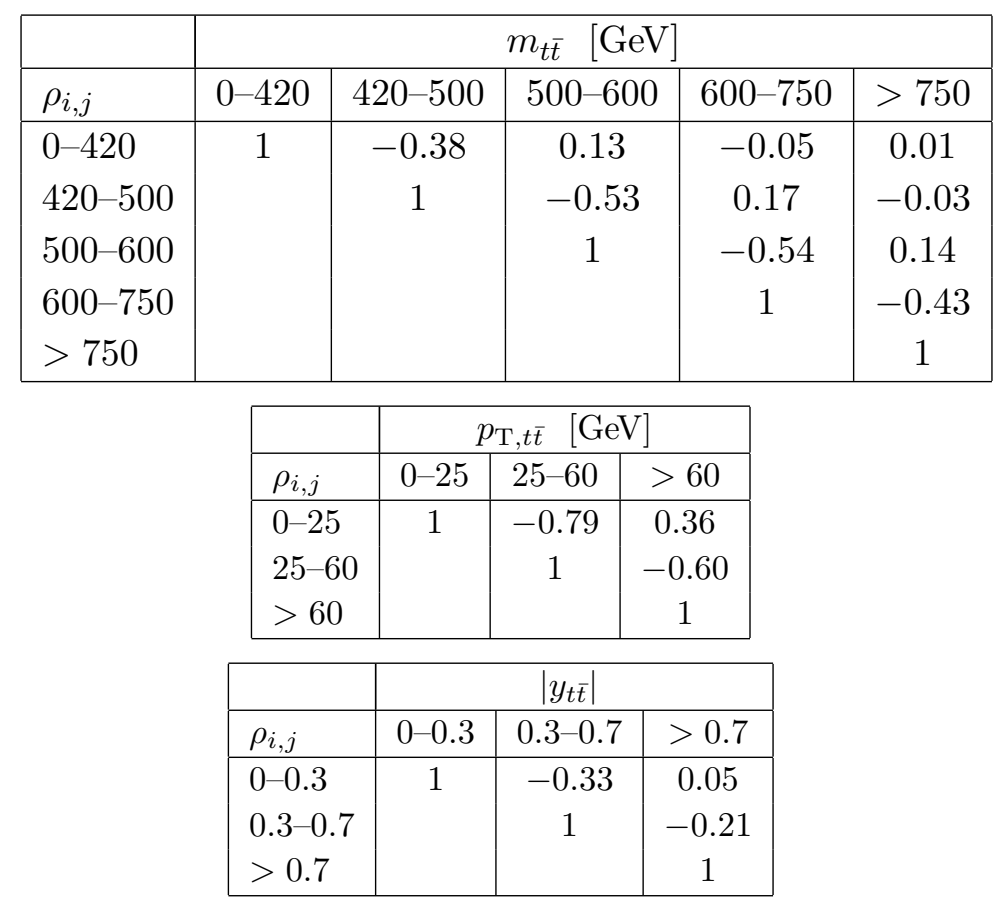

Table 6. Correlation coefficients $\rho_{i, j}$ for the statistical uncertainties between the $i$-th and $j$-th bin of the differential $A_{\mathrm{C}}$ measurement as a function of the $t \bar{t}$ invariant mass, $m_{t \bar{t}}$ (top), the transverse momentum, $p_{\mathrm{T}, t \bar{t}}$ (middle) and the $t \bar{t}$ rapidity, $\left|y_{t \bar{t}}\right|$ (bottom).

\begin{tabular}{|l|c|c|c|c|c|}
\hline & \multicolumn{5}{|c|}{$m_{t \bar{t}}[\mathrm{GeV}]$ for $\beta_{z, t \bar{t}}>0.6$} \\
\hline$A_{\mathrm{C}}$ & $0-420$ & $420-500$ & $500-600$ & $600-750$ & $>750$ \\
\hline Unfolded & $0.054 \pm 0.079$ & $0.008 \pm 0.072$ & $-0.022 \pm 0.075$ & $-0.019 \pm 0.102$ & $0.205 \pm 0.135$ \\
Theory & $0.0145_{-0.0003}^{+0.0005}$ & $0.0213_{-0.0005}^{+0.0006}$ & $0.0240_{-0.0009}^{+0.0003}$ & $0.0280_{-0.0007}^{+0.0012}$ & $0.0607 \pm 0.0002$ \\
\hline
\end{tabular}

Table 7. Measured charge asymmetry, $A_{\mathrm{C}}$, values for the electron and muon channels combined after unfolding as a function of the $t \bar{t}$ invariant mass, $m_{t \bar{t}}$, for $\beta_{z, t \bar{t}}>0.6$. SM predictions, as described in the text, are also reported. The quoted uncertainties include statistical and systematic components after the marginalisation.

We acknowledge the support of ANPCyT, Argentina; YerPhI, Armenia; ARC, Australia; BMWF and FWF, Austria; ANAS, Azerbaijan; SSTC, Belarus; CNPq and FAPESP, Brazil; NSERC, NRC and CFI, Canada; CERN; CONICYT, Chile; CAS, MOST and NSFC, China; COLCIENCIAS, Colombia; MSMT CR, MPO CR and VSC CR, Czech Republic; DNRF, DNSRC and Lundbeck Foundation, Denmark; EPLANET, ERC and NSRF, European Union; IN2P3-CNRS, CEA-DSM/IRFU, France; GNSF, Georgia; BMBF, DFG, HGF, MPG and AvH Foundation, Germany; GSRT and NSRF, Greece; ISF, MINERVA, GIF, DIP and Benoziyo Center, Israel; INFN, Italy; MEXT and JSPS, Japan; CNRST, Morocco; FOM and NWO, Netherlands; BRF and RCN, Norway; MNiSW and NCN, Poland; GRICES and FCT, Portugal; MNE/IFA, Romania; MES of Russia and ROSATOM, Russian Federation; JINR; MSTD, Serbia; MSSR, Slovakia; ARRS and MIZŠ, Slovenia; DST/NRF, South Africa; MINECO, Spain; SRC and Wallenberg Foundation, Sweden; 


\begin{tabular}{|l|c|c|c|c|c|}
\hline & \multicolumn{5}{|c|}{$m_{t \bar{t}}[\mathrm{GeV}]$ for $\beta_{z, t \bar{t}}>0.6$} \\
\hline Source of systematic uncertainty & $0-420$ & $420-500$ & $500-600$ & $600-750$ & $>750$ \\
\hline Lepton reconstruction/identification & $<0.005$ & $<0.005$ & $<0.005$ & $<0.005$ & $<0.005$ \\
Lepton energy scale and resolution & 0.021 & 0.033 & 0.039 & 0.024 & 0.015 \\
Jet energy scale and resolution & 0.014 & 0.026 & 0.061 & 0.095 & 0.111 \\
Missing transverse momentum and pile-up modelling & 0.019 & 0.030 & 0.032 & 0.019 & 0.011 \\
Multi-jets background normalisation & 0.007 & $<0.005$ & $<0.005$ & $<0.005$ & 0.017 \\
b-tagging/mis-tag efficiency & $<0.005$ & $<0.005$ & $<0.005$ & $<0.005$ & $<0.005$ \\
Signal modelling & $<0.005$ & $<0.005$ & $<0.005$ & $<0.005$ & $<0.005$ \\
Parton shower/hadronisation & $<0.005$ & $<0.005$ & $<0.005$ & $<0.005$ & $<0.005$ \\
Monte Carlo sample size & $<0.005$ & $<0.005$ & $<0.005$ & $<0.005$ & $<0.005$ \\
PDF & $<0.005$ & $<0.005$ & $<0.005$ & $<0.005$ & $<0.005$ \\
$W+$ jets normalisation and shape & $<0.005$ & $<0.005$ & $<0.005$ & $<0.005$ & 0.010 \\
\hline Statistical uncertainty & 0.078 & 0.070 & 0.074 & 0.098 & 0.131 \\
\hline
\end{tabular}

Table 8. Systematic uncertainties for the charge asymmetry, $A_{\mathrm{C}}$, measurement for the electron and muon channels combined after unfolding as a function of the $t \bar{t}$ invariant mass, $m_{t \bar{t}}$, for $\beta_{z, t \bar{t}}>$ 0.6. For variations resulting in asymmetric uncertainties, the average absolute deviation from the nominal value is reported. The values reported for each systematic uncertainty are the variation of the mean of posterior probability densities computed considering $1 \sigma$ variations.

\begin{tabular}{|l|c|c|c|c|c|}
\hline & \multicolumn{5}{|c|}{$m_{t \bar{t}}[\mathrm{GeV}]$ for $\beta_{z, t \bar{t}}>0.6$} \\
\hline$\rho_{i, j}$ & $0-420$ & $420-500$ & $500-600$ & $600-750$ & $>750$ \\
\hline $0-420$ & 1 & -0.36 & 0.08 & -0.01 & 0.01 \\
$420-500$ & & 1 & -0.57 & 0.19 & -0.04 \\
$500-600$ & & & 1 & -0.59 & 0.16 \\
$600-750$ & & & & 1 & -0.50 \\
$>750$ & & & & & 1 \\
\hline
\end{tabular}

Table 9. Correlation coefficients $\rho_{i, j}$ for the statistical uncertainties between the $i$-th and $j$-th bin of the differential $A_{\mathrm{C}}$ measurement as a function of the $t \bar{t}$ invariant mass, $m_{t \bar{t}}$, for $\beta_{z, t \bar{t}}>0.6$.

SER, SNSF and Cantons of Bern and Geneva, Switzerland; NSC, Taiwan; TAEK, Turkey; STFC, the Royal Society and Leverhulme Trust, United Kingdom; DOE and NSF, United States of America.

The crucial computing support from all WLCG partners is acknowledged gratefully, in particular from CERN and the ATLAS Tier-1 facilities at TRIUMF (Canada), NDGF (Denmark, Norway, Sweden), CC-IN2P3 (France), KIT/GridKA (Germany), INFN-CNAF (Italy), NL-T1 (Netherlands), PIC (Spain), ASGC (Taiwan), RAL (U.K.) and BNL (U.S.A.) and in the Tier-2 facilities worldwide.

Open Access. This article is distributed under the terms of the Creative Commons Attribution License (CC-BY 4.0), which permits any use, distribution and reproduction in any medium, provided the original author(s) and source are credited. 

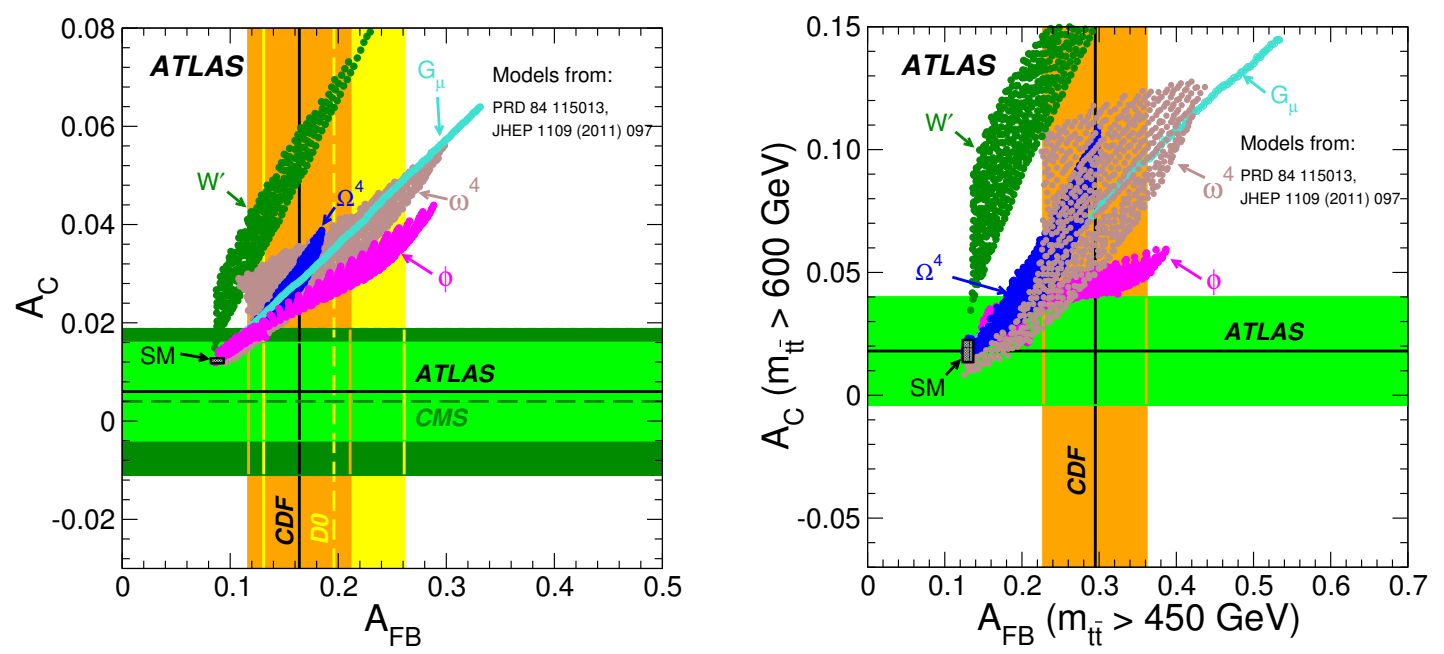

Figure 3. Measured forward-backward asymmetries $A_{\mathrm{FB}}$ at Tevatron and charge asymmetries $A_{\mathrm{C}}$ at LHC, compared with the SM predictions (black box) as well as predictions incorporating various potential new physics contributions (as described in the figure) $[8,60]$. In both plots, where present, the horizontal bands and lines correspond to the ATLAS (light green) and CMS (dark green) measurements, while the vertical ones correspond to the CDF (orange) and D0 (yellow) measurements. The inclusive $A_{\mathrm{C}}$ measurements are reported in the left plot. In the right plot a comparison is reported between the $A_{\mathrm{FB}}$ measurement by $\mathrm{CDF}$ for $m_{t \bar{t}}>450 \mathrm{GeV}$ and the $A_{\mathrm{C}}$ measurement for $m_{t \bar{t}}>600 \mathrm{GeV}$.

\section{References}

[1] S. Jung, A. Pierce and J.D. Wells, Top quark asymmetry from a non-abelian horizontal symmetry, Phys. Rev. D 83 (2011) 114039 [arXiv:1103.4835] [INSPIRE].

[2] O. Antunano, J.H. Kuhn and G. Rodrigo, Top quarks, axigluons and charge asymmetries at hadron colliders, Phys. Rev. D 77 (2008) 014003 [arXiv:0709.1652] [InSPIRE].

[3] A. Djouadi, G. Moreau, F. Richard and R.K. Singh, The forward-backward asymmetry of top quark production at the Tevatron in warped extra dimensional models, Phys. Rev. D 82 (2010) 071702 [arXiv:0906.0604] [INSPIRE].

[4] P. Ferrario and G. Rodrigo, Massive color-octet bosons and the charge asymmetries of top quarks at hadron colliders, Phys. Rev. D 78 (2008) 094018 [arXiv:0809.3354] [INSPIRE].

[5] S. Jung, H. Murayama, A. Pierce and J.D. Wells, Top quark forward-backward asymmetry from new t-channel physics, Phys. Rev. D 81 (2010) 015004 [arXiv:0907.4112] [INSPIRE].

[6] J. Shu, T.M. Tait and K. Wang, Explorations of the top quark forward-backward asymmetry at the Tevatron, Phys. Rev. D 81 (2010) 034012 [arXiv:0911.3237] [InSPIRE].

[7] J. Aguilar-Saavedra and M. Pérez-Victoria, Probing the Tevatron $t \bar{t}$ asymmetry at LHC, JHEP 05 (2011) 034 [arXiv: 1103.2765] [INSPIRE].

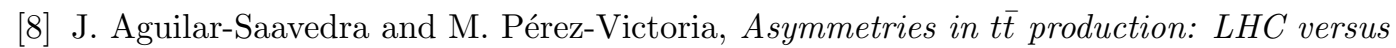
Tevatron, Phys. Rev. D 84 (2011) 115013 [arXiv:1105.4606] [INSPIRE].

[9] I. Dorsner, S. Fajfer, J.F. Kamenik and N. Kosnik, Light colored scalars from grand

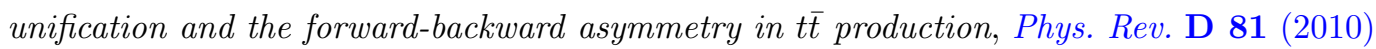
055009 [arXiv:0912.0972] [INSPIRE]. 
[10] B. Grinstein, A.L. Kagan, M. Trott and J. Zupan, Forward-backward asymmetry in $t \bar{t}$ production from flavour symmetries, Phys. Rev. Lett. 107 (2011) 012002 [arXiv:1102.3374] [INSPIRE].

[11] Z. Ligeti, G. Marques Tavares and M. Schmaltz, Explaining the $t \bar{t}$ forward-backward asymmetry without dijet or flavor anomalies, JHEP 06 (2011) 109 [arXiv:1103.2757] [INSPIRE].

[12] P. Ferrario and G. Rodrigo, Constraining heavy colored resonances from top-antitop quark events, Phys. Rev. D 80 (2009) 051701 [arXiv:0906.5541] [INSPIRE].

[13] P.H. Frampton, J. Shu and K. Wang, Axigluon as possible explanation for $p \bar{p} \rightarrow t \bar{t}$ forward-backward asymmetry, Phys. Lett. B 683 (2010) 294 [arXiv:0911.2955] [INSPIRE].

[14] J. Aguilar-Saavedra and M. Pérez-Victoria, Shaping the top asymmetry, Phys. Lett. B 705 (2011) 228 [arXiv: 1107.2120] [INSPIRE].

[15] J.H. Kuhn and G. Rodrigo, Charge asymmetry of heavy quarks at hadron colliders, Phys. Rev. D 59 (1999) 054017 [hep-ph/9807420] [INSPIRE].

[16] J.H. Kuhn and G. Rodrigo, Charge asymmetry in hadroproduction of heavy quarks, Phys. Rev. Lett. 81 (1998) 49 [hep-ph/9802268] [INSPIRE].

[17] W. Bernreuther and Z.-G. Si, Distributions and correlations for top quark pair production and decay at the Tevatron and LHC., Nucl. Phys. B 837 (2010) 90 [arXiv:1003.3926] [InSPIRE].

[18] V. Ahrens, A. Ferroglia, M. Neubert, B.D. Pecjak and L.L. Yang, The top-pair forward-backward asymmetry beyond NLO, Phys. Rev. D 84 (2011) 074004 [arXiv:1106.6051] [INSPIRE].

[19] W. Hollik and D. Pagani, The electroweak contribution to the top quark forward-backward asymmetry at the Tevatron, Phys. Rev. D 84 (2011) 093003 [arXiv:1107.2606] [InSPIRE].

[20] J.H. Kuhn and G. Rodrigo, Charge asymmetries of top quarks at hadron colliders revisited, JHEP 01 (2012) 063 [arXiv:1109.6830] [INSPIRE].

[21] W. Bernreuther and Z.-G. Si, Top quark and leptonic charge asymmetries for the Tevatron and LHC, Phys. Rev. D 86 (2012) 034026 [arXiv:1205.6580] [INSPIRE].

[22] CDF collaboration, T. Aaltonen et al., Forward-backward asymmetry in top quark production in $p \bar{p}$ collisions at sqrts $=1.96$ TeV, Phys. Rev. Lett. 101 (2008) 202001 [arXiv: 0806.2472] [INSPIRE].

[23] CDF collaboration, T. Aaltonen et al., Evidence for a mass dependent forward-backward asymmetry in top quark pair production, Phys. Rev. D 83 (2011) 112003 [arXiv:1101.0034] [INSPIRE].

[24] CDF collaboration, T. Aaltonen et al., Measurement of the top quark forward-backward production asymmetry and its dependence on event kinematic properties, Phys. Rev. D 87 (2013) 092002 [arXiv: 1211.1003] [INSPIRE].

[25] D0 collaboration, V. Abazov et al., First measurement of the forward-backward charge asymmetry in top quark pair production, Phys. Rev. Lett. 100 (2008) 142002 [arXiv: 0712.0851] [INSPIRE].

[26] D0 collaboration, V.M. Abazov et al., Forward-backward asymmetry in top quark-antiquark production, Phys. Rev. D 84 (2011) 112005 [arXiv:1107.4995] [INSPIRE]. 
[27] R. Diener, S. Godfrey and T.A. Martin, Using final state pseudorapidities to improve s-channel resonance observables at the LHC, Phys. Rev. D 80 (2009) 075014 [arXiv:0909.2022] [INSPIRE].

[28] CMS collaboration, Measurement of the charge asymmetry in top-quark pair production in proton-proton collisions at $\sqrt{s}=7 \mathrm{TeV}$, Phys. Lett. B 709 (2012) 28 [arXiv:1112.5100] [INSPIRE].

[29] CMS collaboration, Inclusive and differential measurements of the $t \bar{t}$ charge asymmetry in proton-proton collisions at 7 TeV, Phys. Lett. B 717 (2012) 129 [arXiv:1207.0065] [INSPIRE].

[30] ATLAS collaboration, Measurement of the charge asymmetry in top quark pair production in pp collisions at $\sqrt{s}=7 \mathrm{TeV}$ using the ATLAS detector, Eur. Phys. J. C 72 (2012) 2039 [arXiv: 1203.4211] [INSPIRE].

[31] J. Aguilar-Saavedra and A. Juste, Collider-independent $t \bar{t}$ forward-backward asymmetries, Phys. Rev. Lett. 109 (2012) 211804 [arXiv:1205.1898] [INSPIRE].

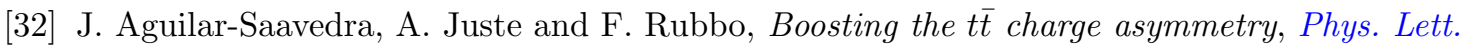
B 707 (2012) 92 [arXiv:1109.3710] [INSPIRE].

[33] ATLAS collaboration, The ATLAS experiment at the CERN Large Hadron Collider, 2008 JINST 3 S08003 [INSPIRE].

[34] ATLAS collaboration, Improved luminosity determination in pp collisions at $\sqrt{s}=7 \mathrm{TeV}$ using the ATLAS detector at the LHC, Eur. Phys. J. C 73 (2013) 2518 [arXiv:1302.4393] [INSPIRE].

[35] M.L. Mangano, M. Moretti, F. Piccinini, R. Pittau and A.D. Polosa, ALPGEN, a generator for hard multiparton processes in hadronic collisions, JHEP 07 (2003) 001 [hep-ph/0206293] [INSPIRE].

[36] J. Pumplin et al., New generation of parton distributions with uncertainties from global QCD analysis, JHEP 07 (2002) 012 [hep-ph/0201195] [INSPIRE].

[37] G. Corcella et al., HERWIG 6: an event generator for hadron emission reactions with interfering gluons (including supersymmetric processes), JHEP 01 (2001) 010 [hep-ph/0011363] [INSPIRE].

[38] J. Butterworth, J.R. Forshaw and M. Seymour, Multiparton interactions in photoproduction at HERA, Z. Phys. C 72 (1996) 637 [hep-ph/9601371] [INSPIRE].

[39] ATLAS collaboration, New ATLAS event generator tunes to 2010 data, ATL-PHYS-PUB-2011-008 (2011).

[40] M. Cacciari, M. Czakon, M. Mangano, A. Mitov and P. Nason, Top-pair production at hadron colliders with next-to-next-to-leading logarithmic soft-gluon resummation, Phys. Lett. B 710 (2012) 612 [arXiv:1111.5869] [INSPIRE].

[41] P. Bärnreuther, M. Czakon and A. Mitov, Percent level precision physics at the Tevatron: first genuine NNLO QCD corrections to $q \bar{q} \rightarrow t \bar{t}+X$, Phys. Rev. Lett. 109 (2012) 132001 [arXiv: 1204.5201] [INSPIRE].

[42] M. Czakon and A. Mitov, NNLO corrections to top-pair production at hadron colliders: the all-fermionic scattering channels, JHEP 12 (2012) 054 [arXiv: 1207.0236] [INSPIRE]. 
[43] M. Czakon and A. Mitov, NNLO corrections to top pair production at hadron colliders: the quark-gluon reaction, JHEP 01 (2013) 080 [arXiv:1210.6832] [INSPIRE].

[44] M. Czakon, P. Fiedler and A. Mitov, The total top quark pair production cross-section at hadron colliders through $O\left(\alpha_{S}^{4}\right)$, Phys. Rev. Lett. 110 (2013) 252004 [arXiv:1303.6254] [INSPIRE].

[45] M. Czakon and A. Mitov, Top++: a program for the calculation of the top-pair cross-section at hadron colliders, arXiv:1112.5675 [INSPIRE].

[46] M. Botje et al., The PDF4LHC working group interim recommendations, arXiv:1101.0538 [INSPIRE].

[47] B.P. Kersevan and E. Richter-Was, The Monte Carlo event generator AcerMC versions 2.0 to 3.8 with interfaces to PYTHIA 6.4, HERWIG 6.5 and ARIADNE 4.1, Comput. Phys. Commun. 184 (2013) 919 [hep-ph/0405247] [INSPIRE].

[48] GEANT4 collaboration, S. Agostinelli et al., GEANT4 - A simulation toolkit, Nucl. Instrum. Meth. A 506 (2003) 250 [inSPIRE].

[49] ATLAS collaboration, The ATLAS simulation infrastructure, Eur. Phys. J. C 70 (2010) 823 [arXiv: 1005.4568] [INSPIRE].

[50] M. Cacciari, G.P. Salam and G. Soyez, The anti- $k_{t}$ jet clustering algorithm, JHEP 04 (2008) 063 [arXiv: 0802.1189] [INSPIRE].

[51] ATLAS collaboration, Jet energy scale and its systematic uncertainty in proton-proton collisions at $\sqrt{s}=7 \mathrm{TeV}$ with ATLAS 2011 data, ATLAS-CONF-2013-004 (2013).

[52] ATLAS collaboration, Commissioning of the ATLAS high-performance b-tagging algorithms in the $7 \mathrm{TeV}$ collision data, ATLAS-CONF-2011-102 (2011).

[53] ATLAS collaboration, Calibrating the b-tag efficiency and mistag rate in $35 \mathrm{pb}^{-1}$ of data with the ATLAS detector, ATLAS-CONF-2011-089 (2011).

[54] ATLAS collaboration, Measurement of the top quark-pair production cross section with ATLAS in pp collisions at $\sqrt{s}=7 \mathrm{TeV}$, Eur. Phys. J. C 71 (2011) 1577 [arXiv:1012.1792] [INSPIRE].

[55] G. Choudalakis, Fully bayesian unfolding, arXiv:1201.4612 [INSPIRE].

[56] G. D'Agostini, A multidimensional unfolding method based on Bayes' theorem, Nucl. Instrum. Meth. A 362 (1995) 487 [INSPIRE].

[57] ATLAS collaboration, Jet energy resolution in proton-proton collisions at $\sqrt{s}=7 \mathrm{TeV}$ recorded in 2010 with the ATLAS detector, Eur. Phys. J. C 73 (2013) 2306 [arXiv: 1210.6210] [INSPIRE].

[58] P. Nason, A new method for combining NLO QCD with shower Monte Carlo algorithms, JHEP 11 (2004) 040 [hep-ph/0409146] [INSPIRE].

[59] S. Frixione, P. Nason and C. Oleari, Matching NLO QCD computations with Parton Shower simulations: the POWHEG method, JHEP 11 (2007) 070 [arXiv:0709.2092] [INSPIRE].

[60] J. Aguilar-Saavedra and M. Pérez-Victoria, Simple models for the top asymmetry: constraints and predictions, JHEP 09 (2011) 097 [arXiv: 1107.0841] [INSPIRE].

[61] J. Aguilar-Saavedra, Single top quark production at LHC with anomalous Wtb couplings, Nucl. Phys. B 804 (2008) 160 [arXiv:0803.3810] [InSPIRE]. 


\section{The ATLAS collaboration}

G. Aad ${ }^{48}$, T. Abajyan ${ }^{21}$, B. Abbott ${ }^{112}$, J. Abdallah ${ }^{12}$, S. Abdel Khalek ${ }^{116}$, O. Abdinov ${ }^{11}$, R. Aben ${ }^{106}$, B. Abi ${ }^{113}$, M. Abolins ${ }^{89}$, O.S. AbouZeid ${ }^{159}$, H. Abramowicz ${ }^{154}$, H. Abreu ${ }^{137}$, Y. Abulaiti ${ }^{147 a, 147 b}$, B.S. Acharya ${ }^{165 a, 165 b, a}$, L. Adamczyk ${ }^{38 a}$, D.L. Adams ${ }^{25}$, T.N. Addy ${ }^{56}$, J. Adelman ${ }^{177}$, S. Adomeit ${ }^{99}$, T. Adye ${ }^{130}$, S. Aefsky ${ }^{23}$, T. Agatonovic-Jovin ${ }^{13 b}$, J.A. Aguilar-Saavedra ${ }^{125 b, b}$, M. Agustoni ${ }^{17}$, S.P. Ahlen ${ }^{22}$, A. Ahmad ${ }^{149}$, F. Ahmadov ${ }^{64, c}$, G. Aielli ${ }^{134 a, 134 b}$, T.P.A. Åkesson ${ }^{80}$, G. Akimoto ${ }^{156}$, A.V. Akimov ${ }^{95}$, M.A. Alam ${ }^{76}$, J. Albert ${ }^{170}$, S. Albrand ${ }^{55}$, M.J. Alconada Verzini ${ }^{70}$, M. Aleksa ${ }^{30}$, I.N. Aleksandrov ${ }^{64}$, F. Alessandria ${ }^{90 a}$, C. Alexa ${ }^{26 a}$, G. Alexander ${ }^{154}$, G. Alexandre ${ }^{49}$, T. Alexopoulos ${ }^{10}$, M. Alhroob ${ }^{165 a, 165 c}$, M. Aliev ${ }^{16}$, G. Alimonti ${ }^{90 a}$, L. Alio ${ }^{84}$, J. Alison ${ }^{31}$, B.M.M. Allbrooke ${ }^{18}$, L.J. Allison ${ }^{71}$, P.P. Allport ${ }^{73}$, S.E. Allwood-Spiers ${ }^{53}$, J. Almond ${ }^{83}$, A. Aloisio ${ }^{103 a, 103 b}$, R. Alon ${ }^{173}$, A. Alonso ${ }^{36}$, F. Alonso ${ }^{70}$, A. Altheimer ${ }^{35}$, B. Alvarez Gonzalez ${ }^{89}$, M.G. Alviggi ${ }^{103 a, 103 b}$, K. Amako ${ }^{65}$,

Y. Amaral Coutinho $24 a$, C. Amelung ${ }^{23}$, V.V. Ammosov ${ }^{129, *}$, S.P. Amor Dos Santos ${ }^{125 a}$, A. Amorim ${ }^{125 a, d}$, S. Amoroso ${ }^{48}$, N. Amram ${ }^{154}$, G. Amundsen ${ }^{23}$, C. Anastopoulos ${ }^{30}$, L.S. Ancu ${ }^{17}$, N. Andari ${ }^{30}$, T. Andeen ${ }^{35}$, C.F. Anders ${ }^{58 b}$, G. Anders ${ }^{58 a}$, K.J. Anderson ${ }^{31}$, A. Andreazza ${ }^{90 a, 90 b}$, V. Andrei ${ }^{58 a}$, X.S. Anduaga ${ }^{70}$, S. Angelidakis ${ }^{9}$, P. Anger ${ }^{44}$, A. Angerami ${ }^{35}$, F. Anghinolfi ${ }^{30}$, A.V. Anisenkov ${ }^{108}$, N. Anjos ${ }^{125 a}$, A. Annovi ${ }^{47}$, A. Antonaki ${ }^{9}$, M. Antonelli ${ }^{47}$, A. Antonov ${ }^{97}$, J. Antos ${ }^{145 b}$, F. Anulli ${ }^{133 a}$, M. Aoki ${ }^{102}$, L. Aperio Bella ${ }^{18}$, R. Apolle ${ }^{119, e}$, G. Arabidze ${ }^{89}$, I. Aracena ${ }^{144}$, Y. Arai ${ }^{65}$, A.T.H. Arce ${ }^{45}$, S. Arfaoui ${ }^{149}$, J-F. Arguin ${ }^{94}$, S. Argyropoulos ${ }^{42}$,

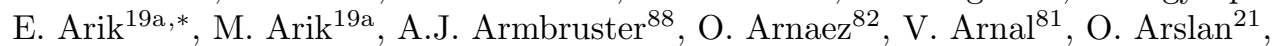

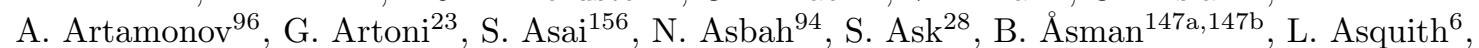
K. Assamagan ${ }^{25}$, R. Astalos ${ }^{145 a}$, A. Astbury ${ }^{170}$, M. Atkinson ${ }^{166}$, N.B. Atlay ${ }^{142}$, B. Auerbach ${ }^{6}$, E. Auge ${ }^{116}$, K. Augsten ${ }^{127}$, M. Aurousseau ${ }^{146 b}$, G. Avolio ${ }^{30}$, G. Azuelos ${ }^{94, f}$, Y. Azuma ${ }^{156}$, M.A. Baak ${ }^{30}$, C. Bacci ${ }^{135 a, 135 b}$, A.M. Bach ${ }^{15}$, H. Bachacou ${ }^{137}$, K. Bachas ${ }^{155}$, M. Backes ${ }^{30}$, M. Backhaus ${ }^{21}$, J. Backus Mayes ${ }^{144}$, E. Badescu ${ }^{26 a}$, P. Bagiacchi133a,133b, P. Bagnaia 133a,133b, Y. Bai ${ }^{33 a}$, D.C. Bailey ${ }^{159}$, T. Bain ${ }^{35}$, J.T. Baines ${ }^{130}$, O.K. Baker ${ }^{177}$, S. Baker ${ }^{77}$, P. Balek ${ }^{128}$, F. Balli ${ }^{137}$, E. Banas ${ }^{39}$, Sw. Banerjee ${ }^{174}$, D. Banfi ${ }^{30}$, A. Bangert ${ }^{151}$, V. Bansal ${ }^{170}$, H.S. Bansil ${ }^{18}$, L. Barak $^{173}$, S.P. Baranov ${ }^{55}$, T. Barber ${ }^{48}$, E.L. Barberio ${ }^{87}$, D. Barberis ${ }^{50 a, 50 b}$, M. Barbero ${ }^{84}$, D.Y. Bardin ${ }^{64}$, T. Barillari ${ }^{100}$, M. Barisonzi ${ }^{176}$, T. Barklow ${ }^{144}$, N. Barlow ${ }^{28}$, B.M. Barnett ${ }^{130}$, R.M. Barnett ${ }^{15}$, A. Baroncelli ${ }^{135 a}$, G. Barone ${ }^{49}$, A.J. Barr ${ }^{119}$, F. Barreiro ${ }^{81}$,

J. Barreiro Guimarães da Costa ${ }^{57}$, R. Bartoldus ${ }^{144}$, A.E. Barton ${ }^{71}$, V. Bartsch ${ }^{150}$, A. Bassalat ${ }^{116}$, A. Basye ${ }^{166}$, R.L. Bates ${ }^{53}$, L. Batkova ${ }^{145 a}$, J.R. Batley ${ }^{28}$, M. Battistin ${ }^{30}$, F. Bauer ${ }^{137}$, H.S. Bawa ${ }^{144, g}$, T. Beau ${ }^{79}$, P.H. Beauchemin ${ }^{162}$, R. Beccherle ${ }^{50 a}$, P. Bechtle ${ }^{21}$, H.P. Beck ${ }^{17}$, K. Becker ${ }^{176}$, S. Becker ${ }^{99}$, M. Beckingham ${ }^{139}$, A.J. Beddall ${ }^{19 c}$, A. Beddall ${ }^{19 c}$, S. Bedikian ${ }^{177}$, V.A. Bednyakov ${ }^{64}$, C.P. Bee ${ }^{84}$, L.J. Beemster ${ }^{106}$, T.A. Beermann ${ }^{176}$, M. Begel ${ }^{25}$, K. Behr ${ }^{119}$, C. Belanger-Champagne ${ }^{86}$, P.J. Bell ${ }^{49}$, W.H. Bell ${ }^{49}$, G. Bella ${ }^{154}$, L. Bellagamba ${ }^{20 a}$, A. Bellerive ${ }^{29}$, M. Bellomo ${ }^{30}$, A. Belloni ${ }^{57}$, O.L. Beloborodova ${ }^{108, h}$, K. Belotskiy ${ }^{97}$, O. Beltramello ${ }^{30}$,

O. Benary ${ }^{154}$, D. Benchekroun ${ }^{136 a}$, K. Bendtz ${ }^{147 a, 147 b}$, N. Benekos ${ }^{166}$, Y. Benhammou ${ }^{154}$, E. Benhar Noccioli ${ }^{49}$, J.A. Benitez Garcia ${ }^{160 b}$, D.P. Benjamin ${ }^{45}$, J.R. Bensinger ${ }^{23}$,

K. Benslama ${ }^{131}$, S. Bentvelsen ${ }^{106}$, D. Berge ${ }^{30}$, E. Bergeaas Kuutmann ${ }^{16}$, N. Berger ${ }^{5}$,

F. Berghaus ${ }^{170}$, E. Berglund ${ }^{106}$, J. Beringer ${ }^{15}$, C. Bernard ${ }^{22}$, P. Bernat ${ }^{77}$, R. Bernhard ${ }^{48}$,

C. Bernius ${ }^{78}$, F.U. Bernlochner ${ }^{170}$, T. Berry ${ }^{76}$, P. Berta ${ }^{128}$, C. Bertella ${ }^{84}$, F. Bertolucci ${ }^{123 a, 123 b}$, M.I. Besana ${ }^{90 a}$, G.J. Besjes ${ }^{105}$, O. Bessidskaia ${ }^{147 a, 147 b}$, N. Besson ${ }^{137}$, S. Bethke ${ }^{100}$, W. Bhimji ${ }^{46}$, R.M. Bianchi ${ }^{124}$, L. Bianchini ${ }^{23}$, M. Bianco ${ }^{30}$, O. Biebel ${ }^{99}$, S.P. Bieniek ${ }^{77}$, K. Bierwagen ${ }^{54}$, J. Biesiada ${ }^{15}$, M. Biglietti ${ }^{135 a}$, J. Bilbao De Mendizabal ${ }^{49}$, H. Bilokon ${ }^{47}$, M. Bindi20a,20b, S. Binet ${ }^{116}$, A. Bingul ${ }^{19 c}$, C. Bini ${ }^{133 a}, 133 b$, B. Bittner ${ }^{100}$, C.W. Black ${ }^{151}$, J.E. Black ${ }^{144}$, K.M. Black ${ }^{22}$, D. Blackburn ${ }^{139}$, R.E. Blair ${ }^{6}$, J.-B. Blanchard ${ }^{137}$, T. Blazek ${ }^{145 a}$, I. Bloch ${ }^{42}$, C. Blocker ${ }^{23}$, J. Blocki ${ }^{39}$, W. Blum ${ }^{82, *}$, U. Blumenschein ${ }^{54}$, G.J. Bobbink ${ }^{106}$, V.S. Bobrovnikov ${ }^{108}$, S.S. Bocchetta ${ }^{80}$, A. Bocci ${ }^{45}$, C.R. Boddy ${ }^{119}$, M. Boehler ${ }^{48}$, J. Boek ${ }^{176}$, T.T. Boek ${ }^{176}$,

N. Boelaert ${ }^{36}$, J.A. Bogaerts ${ }^{30}$, A.G. Bogdanchikov ${ }^{108}$, A. Bogouch ${ }^{91, *}$, C. Bohm ${ }^{147 a}$, J. Bohm ${ }^{126}$, V. Boisvert ${ }^{76}$, T. Bold ${ }^{38 a}$, V. Boldea ${ }^{26 a}$, A.S. Boldyrev ${ }^{98}$, N.M. Bolnet ${ }^{137}$, M. Bomben ${ }^{79}$, M. Bona ${ }^{75}$, M. Boonekamp ${ }^{137}$, S. Bordoni ${ }^{79}$, C. Borer ${ }^{17}$, A. Borisov ${ }^{129}$, G. Borissov ${ }^{71}$, M. Borri ${ }^{83}$, 


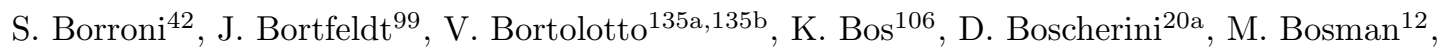
H. Boterenbrood ${ }^{106}$, J. Bouchami ${ }^{94}$, J. Boudreau ${ }^{124}$, E.V. Bouhova-Thacker ${ }^{71}$, D. Boumediene ${ }^{34}$, C. Bourdarios ${ }^{116}$, N. Bousson ${ }^{84}$, S. Boutouil ${ }^{136 \mathrm{~d}}$, A. Boveia ${ }^{31}$, J. Boyd ${ }^{30}$, I.R. Boyko ${ }^{64}$, I. Bozovic-Jelisavcic ${ }^{13 b}$, J. Bracinik ${ }^{18}$, P. Branchini ${ }^{135 a}$, A. Brandt ${ }^{8}$, G. Brandt ${ }^{15}$, O. Brandt ${ }^{54}$, U. Bratzler ${ }^{157}$, B. Brau ${ }^{85}$, J.E. Brau ${ }^{115}$, H.M. Braun ${ }^{176, *}$, S.F. Brazzale ${ }^{165 a, 165 c}$, B. Brelier ${ }^{159}$, K. Brendlinger ${ }^{121}$, R. Brenner ${ }^{167}$, S. Bressler ${ }^{173}$, T.M. Bristow ${ }^{46}$, D. Britton ${ }^{53}$, F.M. Brochu ${ }^{28}$, I. Brock ${ }^{21}$, R. Brock ${ }^{89}$, F. Broggi ${ }^{90 a}$, C. Bromberg ${ }^{89}$, J. Bronner ${ }^{100}$, G. Brooijmans ${ }^{35}$, T. Brooks ${ }^{76}$, W.K. Brooks ${ }^{32 b}$, J. Brosamer ${ }^{15}$, E. Brost ${ }^{115}$, G. Brown ${ }^{83}$, J. Brown ${ }^{55}$, P.A. Bruckman de Renstrom ${ }^{39}$, D. Bruncko ${ }^{145 b}$, R. Bruneliere ${ }^{48}$, S. Brunet ${ }^{60}$, A. Bruni ${ }^{20 a}$, G. Bruni ${ }^{20 a}$, M. Bruschi ${ }^{20 a}$, L. Bryngemark ${ }^{80}$, T. Buanes ${ }^{14}$, Q. Buat ${ }^{55}$, F. Bucci $^{49}$, J. Buchanan ${ }^{119}$, P. Buchholz ${ }^{142}$, R.M. Buckingham ${ }^{119}$, A.G. Buckley ${ }^{46}$, S.I. Buda ${ }^{26 a}$, I.A. Budagov ${ }^{64}$, B. Budick ${ }^{109}$, F. Buehrer ${ }^{48}$, L. Bugge ${ }^{118}$, O. Bulekov ${ }^{97}$, A.C. Bundock ${ }^{73}$, M. Bunse ${ }^{43}$, H. Burckhart ${ }^{30}$, S. Burdin ${ }^{73}$, T. Burgess ${ }^{14}$, S. Burke ${ }^{130}$, I. Burmeister ${ }^{43}$, E. Busato ${ }^{34}$, V. Büscher ${ }^{82}$, P. Bussey ${ }^{53}$, C.P. Buszello ${ }^{167}$, B. Butler ${ }^{57}$, J.M. Butler ${ }^{22}$, A.I. Butt ${ }^{3}$, C.M. Buttar ${ }^{53}$, J.M. Butterworth ${ }^{77}$, W. Buttinger ${ }^{28}$, A. Buzatu ${ }^{53}$, M. Byszewski ${ }^{10}$,

S. Cabrera Urbán ${ }^{168}$, D. Caforio ${ }^{20 a, 20 b}$, O. Cakir ${ }^{4 a}$, P. Calafiura ${ }^{15}$, G. Calderini ${ }^{79}$, P. Calfayan ${ }^{99}$, R. Calkins ${ }^{107}$, L.P. Caloba ${ }^{24 a}$, R. Caloi ${ }^{133 a, 133 b}$, D. Calvet ${ }^{34}$, S. Calvet ${ }^{34}$, R. Camacho Toro ${ }^{49}$, P. Camarri ${ }^{134 a, 134 b}$, D. Cameron ${ }^{118}$, L.M. Caminada ${ }^{15}$, R. Caminal Armadans ${ }^{12}$, S. Campana ${ }^{30}$, M. Campanelli ${ }^{77}$, V. Canale ${ }^{103 a, 103 b}$, F. Canelli ${ }^{31}$, A. Canepa ${ }^{160 a}$, J. Cantero ${ }^{81}$, R. Cantrill ${ }^{76}$,

T. $\mathrm{Cao}^{40}$, M.D.M. Capeans Garrido ${ }^{30}$, I. Caprini ${ }^{26 a}$, M. Caprini ${ }^{26 a}$, M. Capua ${ }^{37 a, 37 b}$,

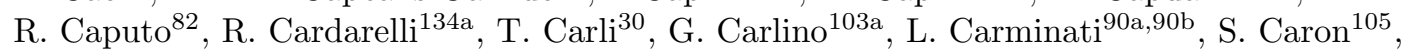

E. Carquin ${ }^{32 \mathrm{a}}$, G.D. Carrillo-Montoya ${ }^{146 c}$, A.A. Carter ${ }^{75}$, J.R. Carter ${ }^{28}$, J. Carvalho ${ }^{125 a, i}$,

D. Casadei ${ }^{77}$, M.P. Casado ${ }^{12}$, C. Caso ${ }^{50 a, 50 b, *}$, E. Castaneda-Miranda ${ }^{146 b}$, A. Castelli ${ }^{106}$,

V. Castillo Gimenez ${ }^{168}$, N.F. Castro ${ }^{125 a}$, P. Catastini ${ }^{57}$, A. Catinaccio ${ }^{30}$, J.R. Catmore $^{71}$,

A. Cattai $^{30}$, G. Cattani ${ }^{134 a, 134 b}$, S. Caughron ${ }^{89}$, V. Cavaliere ${ }^{166}$, D. Cavalli ${ }^{90 a}$,

M. Cavalli-Sforza ${ }^{12}$, V. Cavasinni ${ }^{123 a, 123 b}$, F. Ceradini ${ }^{135 a, 135 b}$, B. Cerio ${ }^{45}$, A.S. Cerqueira ${ }^{24 b}$,

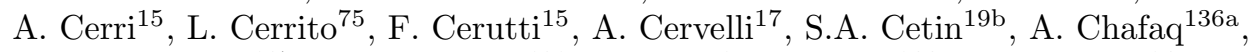

D. Chakraborty ${ }^{107}$, I. Chalupkova ${ }^{128}$, K. Chan $^{3}$, P. Chang ${ }^{166}$, B. Chapleau ${ }^{86}$, J.D. Chapman ${ }^{28}$,

D. Charfeddine ${ }^{116}$, D.G. Charlton ${ }^{18}$, V. Chavda ${ }^{83}$, C.A. Chavez Barajas ${ }^{30}$, S. Cheatham ${ }^{86}$,

S. Chekanov ${ }^{6}$, S.V. Chekulaev ${ }^{160 a}$, G.A. Chelkov ${ }^{64}$, M.A. Chelstowska ${ }^{88}$, C. Chen ${ }^{63}$, H. Chen ${ }^{25}$, K. Chen ${ }^{149}$, S. Chen ${ }^{33 c}$, X. Chen ${ }^{174}$, Y. Chen ${ }^{35}$, Y. Cheng ${ }^{31}$, A. Cheplakov ${ }^{64}$,

R. Cherkaoui El Moursli ${ }^{136 e}$, V. Chernyatin ${ }^{25, *}$, E. Cheu ${ }^{7}$, L. Chevalier ${ }^{137}$, V. Chiarella ${ }^{47}$, G. Chiefari ${ }^{103 a, 103 b}$, J.T. Childers ${ }^{30}$, A. Chilingarov ${ }^{71}$, G. Chiodini ${ }^{72 a}$, A.S. Chisholm ${ }^{18}$,

R.T. Chislett ${ }^{77}$, A. Chitan ${ }^{26 a}$, M.V. Chizhov ${ }^{64}$, G. Choudalakis ${ }^{31}$, S. Chouridou ${ }^{9}$, B.K.B. Chow ${ }^{99}$, I.A. Christidi 77 , D. Chromek-Burckhart ${ }^{30}$, M.L. Chu ${ }^{152}$, J. Chudoba ${ }^{126}$, G. Ciapetti ${ }^{133 a, 133 b}$, A.K. Ciftci ${ }^{4 a}$, R. Ciftci ${ }^{4 a}$, D. Cinca ${ }^{62}$, V. Cindro ${ }^{74}$, A. Ciocio $^{15}$, M. Cirilli ${ }^{88}$, P. Cirkovic ${ }^{13 b}$, Z.H. Citron ${ }^{173}$, M. Citterio ${ }^{90 a}$, M. Ciubancan ${ }^{26 a}$, A. Clark ${ }^{49}$, P.J. Clark ${ }^{46}$, R.N. Clarke ${ }^{15}$, W. Cleland ${ }^{124}$, J.C. Clemens ${ }^{84}$, B. Clement ${ }^{55}$, C. Clement ${ }^{147 a, 147 b}$, Y. Coadou ${ }^{84}$, M. Cobal ${ }^{165 a, 165 c}$, A. Coccaro ${ }^{139}$, J. Cochran ${ }^{63}$, S. Coelli ${ }^{90 a}$, L. Coffey $^{23}$, J.G. Cogan ${ }^{144}$, J. Coggeshall ${ }^{166}$, J. Colas ${ }^{5}$, B. Cole ${ }^{35}$, S. Cole ${ }^{107}$, A.P. Colijn ${ }^{106}$, C. Collins-Tooth ${ }^{53}$, J. Collot ${ }^{55}$, T. Colombo ${ }^{58 c}$, G. Colon ${ }^{85}$, G. Compostella ${ }^{100}$, P. Conde Muiño ${ }^{125 a}$, E. Coniavitis ${ }^{167}$, M.C. Conidi ${ }^{12}$, S.M. Consonni90a,90b , V. Consorti ${ }^{48}$, S. Constantinescu ${ }^{26 a}$, C. Conta ${ }^{120 a, 120 b}$, G. Conti ${ }^{57}$, F. Conventi ${ }^{103 a}, j$, M. Cooke ${ }^{15}$, B.D. Cooper ${ }^{77}$, A.M. Cooper-Sarkar ${ }^{119}$,

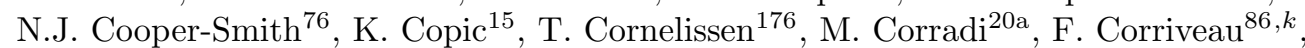

A. Corso-Radu ${ }^{164}$, A. Cortes-Gonzalez ${ }^{12}$, G. Cortiana ${ }^{100}$, G. Costa ${ }^{90 a}$, M.J. Costa ${ }^{168}$,

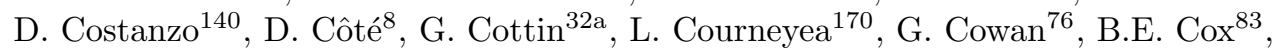

K. Cranmer ${ }^{109}$, G. Cree ${ }^{29}$, S. Crépé-Renaudin ${ }^{55}$, F. Crescioli ${ }^{79}$, M. Crispin Ortuzar ${ }^{119}$,

M. Cristinziani ${ }^{21}$, G. Crosetti ${ }^{37 a}, 37 b$, C.-M. Cuciuc ${ }^{26 a}$, C. Cuenca Almenar ${ }^{177}$,

T. Cuhadar Donszelmann ${ }^{140}$, J. Cummings ${ }^{177}$, M. Curatolo ${ }^{47}$, C. Cuthbert ${ }^{151}$, H. Czirr ${ }^{142}$,

P. Czodrowski ${ }^{44}$, Z. Czyczula ${ }^{177}$, S. D'Auria ${ }^{53}$, M. D'Onofrio ${ }^{73}$, A. D’Orazio $^{133 a, 133 b}$,

M.J. Da Cunha Sargedas De Sousa ${ }^{125 a}$, C. Da Via ${ }^{83}$, W. Dabrowski ${ }^{38 a}$, A. Dafinca ${ }^{19}$, T. Dai ${ }^{88}$, F. Dallaire ${ }^{94}$, C. Dallapiccola ${ }^{85}$, M. Dam $^{36}$, D.S. Damiani ${ }^{138}$, A.C. Daniells ${ }^{18}$, 
M. Dano Hoffmann ${ }^{36}$, V. Dao ${ }^{105}$, G. Darbo ${ }^{50 a}$, G.L. Darlea ${ }^{26 c}$, S. Darmora ${ }^{8}$, J.A. Dassoulas ${ }^{42}$, W. Davey ${ }^{21}$, C. David ${ }^{170}$, T. Davidek ${ }^{128}$, E. Davies ${ }^{119, e}$, M. Davies ${ }^{94}$, O. Davignon $^{79}$, A.R. Davison ${ }^{77}$, Y. Davygora ${ }^{58 a}$, E. Dawe ${ }^{143}$, I. Dawson ${ }^{140}$, R.K. Daya-Ishmukhametova ${ }^{23}$, K. De ${ }^{8}$, R. de Asmundis ${ }^{103 a}$, S. De Castro ${ }^{20 a, 20 b}$, S. De Cecco ${ }^{79}$, J. de Graat ${ }^{99}$, N. De Groot ${ }^{105}$ P. de Jong ${ }^{106}$, C. De La Taille ${ }^{116}$, H. De la Torre ${ }^{81}$, F. De Lorenzi ${ }^{63}$, L. De Nooij ${ }^{106}$, D. De Pedis ${ }^{133 a}$, A. De Salvo ${ }^{133 a}$, U. De Sanctis ${ }^{165 a, 165 c}$, A. De Santo ${ }^{150}$, J.B. De Vivie De Regie ${ }^{116}$, G. De Zorzi ${ }^{133 a, 133 b}$, W.J. Dearnaley ${ }^{71}$, R. Debbe ${ }^{25}$, C. Debenedetti ${ }^{46}$, B. Dechenaux ${ }^{55}$, D.V. Dedovich ${ }^{64}$, J. Degenhardt ${ }^{121}$, J. Del Peso ${ }^{81}$, T. Del Prete ${ }^{123 a, 123 b}$, T. Delemontex ${ }^{55}$, F. Deliot ${ }^{137}$, M. Deliyergiyev ${ }^{74}$, A. Dell'Acqua ${ }^{30}$, L. Dell'Asta ${ }^{22}$, M. Della Pietra ${ }^{103 a, j}$, D. della Volpe ${ }^{49}$, M. Delmastro ${ }^{5}$, P.A. Delsart ${ }^{55}$, C. Deluca ${ }^{106}$, S. Demers ${ }^{177}$, M. Demichev ${ }^{64}$, A. Demilly ${ }^{79}$, B. Demirkoz ${ }^{12, l}$, S.P. Denisov ${ }^{129}$, D. Derendarz ${ }^{39}$, J.E. Derkaouii ${ }^{136 d}$, F. Derue ${ }^{79}$, P. Dervan ${ }^{73}$, K. Desch ${ }^{21}$, P.O. Deviveiros ${ }^{106}$, A. Dewhurst ${ }^{130}$, B. DeWilde ${ }^{149}$, S. Dhaliwal ${ }^{106}$, R. Dhullipudi ${ }^{78, m}$, A. Di Ciaccio ${ }^{134 a, 134 b}$, L. Di Ciaccio ${ }^{5}$, C. Di Donato 103a,103b, A. Di Girolamo ${ }^{30}$, B. Di Girolamo ${ }^{30}$, A. Di Mattia ${ }^{153}$, B. Di Micco ${ }^{135 a, 135 b}$, R. Di Nardo ${ }^{47}$, A. Di Simone ${ }^{48}$, R. Di Sipio ${ }^{20 a, 20 b}$, D. Di Valentino ${ }^{29}$, M.A. Diaz ${ }^{32}$, E.B. Diehl ${ }^{88}$, J. Dietrich ${ }^{42}$, T.A. Dietzsch ${ }^{58 a}$, S. Diglio ${ }^{87}$, K. Dindar Yagci $^{40}$, J. Dingfelder ${ }^{21}$, C. Dionisi ${ }^{133 a, 133 b}$,

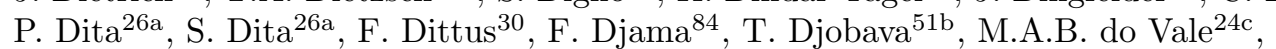

A. Do Valle Wemans ${ }^{125 a, n}$, T.K.O. Doan ${ }^{5}$, D. Dobos ${ }^{30}$, E. Dobson ${ }^{77}$, J. Dodd ${ }^{35}$, C. Doglioni ${ }^{49}$, T. Doherty ${ }^{53}$, T. Dohmae ${ }^{156}$, Y. Doi ${ }^{65, *}$, J. Dolejsi $^{128}$, Z. Dolezal ${ }^{128}$, B.A. Dolgoshein ${ }^{97, *}$,

M. Donadelli24d, S. Donati ${ }^{123 a, 123 b}$, J. Donini ${ }^{34}$, J. Dopke ${ }^{30}$, A. Doria ${ }^{103 a}$, A. Dos Anjos ${ }^{174}$,

A. Dotti ${ }^{123 a, 123 b}$, M.T. Dova ${ }^{70}$, A.T. Doyle ${ }^{53}$, M. Dris ${ }^{10}$, J. Dubbert ${ }^{88}$, S. Dube $^{15}$, E. Dubreuil ${ }^{34}$, E. Duchovni ${ }^{173}$, G. Duckeck ${ }^{99}$, O.A. Ducu ${ }^{26 a}$, D. Duda ${ }^{176}$, A. Dudarev ${ }^{30}$, F. Dudziak ${ }^{63}$,

L. Duflot ${ }^{116}$, L. Duguid ${ }^{76}$, M. Dührssen ${ }^{30}$, M. Dunford ${ }^{58 a}$, H. Duran Yildiz ${ }^{4 a}$, M. Düren ${ }^{52}$, M. Dwuznik ${ }^{38 a}$, J. Ebke ${ }^{99}$, W. Edson ${ }^{2}$, C.A. Edwards ${ }^{76}$, N.C. Edwards ${ }^{46}$, W. Ehrenfeld ${ }^{21}$, T. Eifert ${ }^{144}$, G. Eigen $^{14}$, K. Einsweiler ${ }^{15}$, E. Eisenhandler ${ }^{75}$, T. Ekelof ${ }^{167}$, M. El Kacimi ${ }^{136 c}$, M. Ellert ${ }^{167}$, S. Elles ${ }^{5}$, F. Ellinghaus ${ }^{82}$, K. Ellis ${ }^{75}$, N. Ellis $^{30}$, J. Elmsheuser ${ }^{99}$, M. Elsing ${ }^{30}$,

D. Emeliyanov ${ }^{130}$, Y. Enari ${ }^{156}$, O.C. Endner ${ }^{82}$, M. Endo ${ }^{117}$, R. Engelmann ${ }^{149}$, J. Erdmann ${ }^{177}$, A. Ereditato ${ }^{17}$, D. Eriksson ${ }^{147 a}$, G. Ernis ${ }^{176}$, J. Ernst ${ }^{2}$, M. Ernst ${ }^{25}$, J. Ernwein ${ }^{137}$, D. Errede ${ }^{166}$, S. Errede ${ }^{166}$, E. Ertel ${ }^{82}$, M. Escalier ${ }^{116}$, H. Esch ${ }^{43}$, C. Escobar ${ }^{124}$, X. Espinal Curull ${ }^{12}$, B. Esposito ${ }^{47}$, F. Etienne ${ }^{84}$, A.I. Etienvre ${ }^{137}$, E. Etzion ${ }^{154}$, D. Evangelakou ${ }^{54}$, H. Evans ${ }^{60}$, L. Fabbri ${ }^{20 a}$,20b , G. Facini ${ }^{30}$, R.M. Fakhrutdinov ${ }^{129}$, S. Falciano ${ }^{133 a}$, Y. Fang ${ }^{33 a}$, M. Fanti ${ }^{90 a}{ }^{90 b}$, A. Farbin ${ }^{8}$, A. Farilla ${ }^{135 a}$, T. Farooque ${ }^{159}$, S. Farrell ${ }^{164}$, S.M. Farrington ${ }^{171}$, P. Farthouat ${ }^{30}$, F. Fassi ${ }^{168}$, P. Fassnacht ${ }^{30}$, D. Fassouliotis ${ }^{9}$, B. Fatholahzadeh ${ }^{159}$, A. Favareto ${ }^{50 a, 50 b}$,

L. Fayard ${ }^{116}$, P. Federic ${ }^{145 a}$, O.L. Fedin ${ }^{122}$, W. Fedorko ${ }^{169}$, M. Fehling-Kaschek ${ }^{48}$, L. Feligioni ${ }^{84}$, C. Feng ${ }^{33 d}$, E.J. Feng ${ }^{6}$, H. Feng ${ }^{88}$, A.B. Fenyuk ${ }^{129}$, W. Fernando ${ }^{6}$, S. Ferrag ${ }^{53}$, J. Ferrando ${ }^{53}$, V. Ferrara ${ }^{42}$, A. Ferrari ${ }^{167}$, P. Ferrari ${ }^{106}$, R. Ferrari ${ }^{120 a}$, D.E. Ferreira de Lima ${ }^{53}$, A. Ferrer ${ }^{168}$ D. Ferrere ${ }^{49}$, C. Ferretti ${ }^{88}$, A. Ferretto Parodi ${ }^{50 a, 50 b}$, M. Fiascaris ${ }^{31}$, F. Fiedler ${ }^{82}$, A. Filipčic ${ }^{74}$, M. Filipuzzi ${ }^{42}$, F. Filthaut ${ }^{105}$, M. Fincke-Keeler ${ }^{170}$, K.D. Finelli ${ }^{45}$, M.C.N. Fiolhais ${ }^{125 a, i}$, L. Fiorini ${ }^{168}$, A. Firan ${ }^{40}$, J. Fischer ${ }^{176}$, M.J. Fisher ${ }^{110}$, E.A. Fitzgerald ${ }^{23}$, M. Flechl ${ }^{48}$, I. Fleck ${ }^{142}$, P. Fleischmann ${ }^{175}$, S. Fleischmann ${ }^{176}$, G.T. Fletcher ${ }^{140}$, G. Fletcher ${ }^{75}$, T. Flick ${ }^{176}$, A. Floderus ${ }^{80}$, L.R. Flores Castillo ${ }^{174}$, A.C. Florez Bustos ${ }^{160 b}$, M.J. Flowerdew ${ }^{100}$, T. Fonseca Martin ${ }^{17}$, A. Formica ${ }^{137}$, A. Forti ${ }^{83}$, D. Fortin ${ }^{160 a}$, D. Fournier ${ }^{116}$, H. Fox $^{71}$, P. Francavilla ${ }^{12}$, M. Franchini ${ }^{20 a}$,20b , S. Franchino ${ }^{30}$, D. Francis ${ }^{30}$, M. Franklin ${ }^{57}$, S. Franz ${ }^{61}$, M. Fraternali ${ }^{120 a, 120 b}$, S. Fratina ${ }^{121}$, S.T. French ${ }^{28}$, C. Friedrich ${ }^{42}$, F. Friedrich ${ }^{44}$, D. Froidevaux ${ }^{30}$, J.A. Frost ${ }^{28}$, C. Fukunaga ${ }^{157}$, E. Fullana Torregrosa ${ }^{128}$, B.G. Fulsom ${ }^{144}$, J. Fuster ${ }^{168}$, C. Gabaldon ${ }^{55}$,

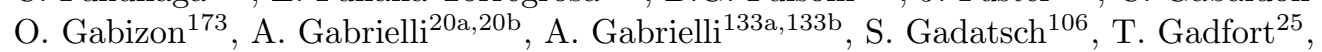
S. Gadomski ${ }^{49}$, G. Gagliardi ${ }^{50 a, 50 b}$, P. Gagnon ${ }^{60}$, C. Galea ${ }^{99}$, B. Galhardo ${ }^{125 a}$, E.J. Gallas ${ }^{119}$, V. Gallo ${ }^{17}$, B.J. Gallop ${ }^{130}$, P. Gallus ${ }^{127}$, G. Galster ${ }^{36}$, K.K. Gan ${ }^{110}$, R.P. Gandrajula ${ }^{62}$, J. Gao ${ }^{33 b, o}$, Y.S. Gao ${ }^{144, g}$, F.M. Garay Walls ${ }^{46}$, F. Garberson ${ }^{177}$, C. García ${ }^{168}$, J.E. García Navarro ${ }^{168}$, M. Garcia-Sciveres ${ }^{15}$, R.W. Gardner ${ }^{31}$, N. Garelli ${ }^{144}$, V. Garonne ${ }^{30}$, C. Gatti ${ }^{47}$, G. Gaudio ${ }^{120 a}$, B. Gaur ${ }^{142}$, L. Gauthier ${ }^{94}$, P. Gauzzi1 ${ }^{133 a, 133 b}$, I.L. Gavrilenko ${ }^{95}$,

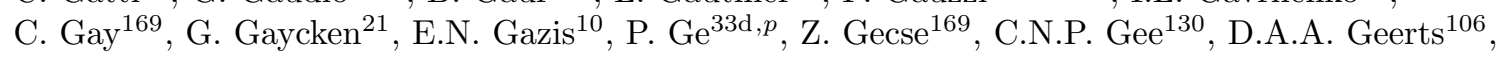


Ch. Geich-Gimbel ${ }^{21}$, K. Gellerstedt ${ }^{147 a, 147 b}$, C. Gemme ${ }^{50 a}$, A. Gemmell ${ }^{53}$, M.H. Genest ${ }^{55}$, S. Gentile ${ }^{133 a, 133 b}$, M. George ${ }^{54}$, S. George ${ }^{76}$, D. Gerbaudo ${ }^{164}$, A. Gershon ${ }^{154}$, H. Ghazlane ${ }^{136 b}$, N. Ghodbane ${ }^{34}$, B. Giacobbe ${ }^{20 a}$, S. Giagu ${ }^{133 a, 133 b}$, V. Giangiobbe ${ }^{12}$, P. Giannetti ${ }^{123 a, 123 b}$, F. Gianotti ${ }^{30}$, B. Gibbard ${ }^{25}$, S.M. Gibson ${ }^{76}$, M. Gilchriese ${ }^{15}$, T.P.S. Gillam ${ }^{28}$, D. Gillberg ${ }^{30}$, A.R. Gillman ${ }^{130}$, D.M. Gingrich ${ }^{3, f}$, N. Giokaris ${ }^{9}$, M.P. Giordani ${ }^{165 a, 165 c}$, R. Giordano ${ }^{103 a, 103 b}$, F.M. Giorgi ${ }^{16}$, P. Giovannini ${ }^{100}$, P.F. Giraud ${ }^{137}$, D. Giugni ${ }^{90 a}$, C. Giuliani ${ }^{48}$, M. Giunta ${ }^{94}$, B.K. Gjelsten ${ }^{118}$, I. Gkialas ${ }^{155, q}$, L.K. Gladilin ${ }^{98}$, C. Glasman ${ }^{81}$, J. Glatzer ${ }^{21}$, A. Glazov ${ }^{42}$, G.L. Glonti ${ }^{64}$, M. Goblirsch-Kolb ${ }^{100}$, J.R. Goddard ${ }^{75}$, J. Godfrey ${ }^{143}$, J. Godlewski ${ }^{30}$, C. Goeringer ${ }^{82}$, S. Goldfarb ${ }^{88}$, T. Golling ${ }^{177}$, D. Golubkov ${ }^{129}$, A. Gomes ${ }^{125 a, d}$, L.S. Gomez Fajardo ${ }^{42}$, R. Gonçalo ${ }^{76}$, J. Goncalves Pinto Firmino Da Costa ${ }^{42}$, L. Gonella ${ }^{21}$, S. González de la Hoz ${ }^{168}$, G. Gonzalez Parra ${ }^{12}$, M.L. Gonzalez Silva ${ }^{27}$, S. Gonzalez-Sevilla ${ }^{49}$, J.J. Goodson ${ }^{149}$, L. Goossens ${ }^{30}$, P.A. Gorbounov ${ }^{96}$, H.A. Gordon ${ }^{25}$, I. Gorelov ${ }^{104}$, G. Gorfine ${ }^{176}$, B. Gorini ${ }^{30}$, E. Gorini ${ }^{72 a, 72 b}$, A. Gorišek ${ }^{74}$, E. Gornicki ${ }^{39}$, A.T. Goshaw ${ }^{6}$, C. Gössling ${ }^{43}$, M.I. Gostkin ${ }^{64}$, I. Gough Eschrich ${ }^{164}$, M. Gouighri ${ }^{136 a}$, D. Goujdami ${ }^{136 c}$, M.P. Goulette ${ }^{49}$, A.G. Goussiou ${ }^{139}$, C. Goy ${ }^{5}$, S. Gozpinar ${ }^{23}$, H.M.X. Grabas ${ }^{137}$, L. Graber ${ }^{54}$, I. Grabowska-Bold ${ }^{38 a}$,

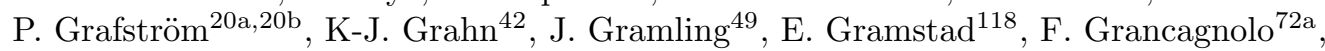
S. Grancagnolo ${ }^{16}$, V. Grassi ${ }^{149}$, V. Gratchev ${ }^{122}$, H.M. Gray ${ }^{30}$, J.A. Gray ${ }^{149}$, E. Graziani ${ }^{135 a}$, O.G. Grebenyuk ${ }^{122}$, Z.D. Greenwood ${ }^{78, m}$, K. Gregersen ${ }^{36}$, I.M. Gregor ${ }^{42}$, P. Grenier ${ }^{144}$, J. Griffiths ${ }^{8}$, N. Grigalashvili ${ }^{64}$, A.A. Grillo ${ }^{138}$, K. Grimm ${ }^{71}$, S. Grinstein ${ }^{12, r}$, Ph. Gris ${ }^{34}$, Y.V. Grishkevich ${ }^{98}$, J.-F. Grivaz ${ }^{116}$, J.P. Grohs ${ }^{44}$, A. Grohsjean ${ }^{42}$, E. Gross ${ }^{173}$,

J. Grosse-Knetter ${ }^{54}$, G.C. Grossi ${ }^{134 a, 134 b}$, J. Groth-Jensen ${ }^{173}$, Z.J. Grout ${ }^{150}$, K. Grybel ${ }^{142}$, F. Guescini ${ }^{49}$, D. Guest ${ }^{177}$, O. Gueta ${ }^{154}$, C. Guicheney ${ }^{34}$, E. Guido ${ }^{50 a, 50 b}$, T. Guillemin ${ }^{116}$ S. Guindon ${ }^{2}$, U. Gul ${ }^{53}$, C. Gumpert ${ }^{44}$, J. Gunther ${ }^{127}$, J. Guo ${ }^{35}$, S. Gupta ${ }^{119}$, P. Gutierrez ${ }^{112}$, N.G. Gutierrez Ortiz ${ }^{53}$, C. Gutschow ${ }^{77}$, N. Guttman ${ }^{154}$, C. Guyot ${ }^{137}$, C. Gwenlan ${ }^{119}$, C.B. Gwilliam ${ }^{73}$, A. Haas ${ }^{109}$, C. Haber ${ }^{15}$, H.K. Hadavand ${ }^{8}$, P. Haefner ${ }^{21}$, S. Hageboeck ${ }^{21}$, Z. Hajduk ${ }^{39}$, H. Hakobyan ${ }^{178}$, M. Haleem ${ }^{41}$, D. Hall ${ }^{119}$, G. Halladjian ${ }^{62}$, K. Hamacher ${ }^{176}$, P. Hamal ${ }^{114}$, K. Hamano ${ }^{87}$, M. Hamer ${ }^{54}$, A. Hamilton ${ }^{146 a, s}$, S. Hamilton ${ }^{162}$, L. Han ${ }^{33 b}$, K. Hanagaki ${ }^{117}$, K. Hanawa ${ }^{156}$, M. Hance ${ }^{15}$, C. Handel ${ }^{82}$, P. Hanke ${ }^{58 a}$, J.R. Hansen ${ }^{36}$,

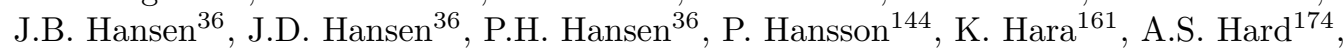
T. Harenberg ${ }^{176}$, S. Harkusha ${ }^{91}$, D. Harper ${ }^{88}$, R.D. Harrington ${ }^{46}$, O.M. Harris ${ }^{139}$, P.F. Harrison ${ }^{171}$, F. Hartjes ${ }^{106}$, A. Harvey ${ }^{56}$, S. Hasegawa ${ }^{102}$, Y. Hasegawa ${ }^{141}$, S. Hassani ${ }^{137}$, S. Haug ${ }^{17}$, M. Hauschild ${ }^{30}$, R. Hauser ${ }^{89}$, M. Havranek ${ }^{21}$, C.M. Hawkes ${ }^{18}$, R.J. Hawkings ${ }^{30}$, A.D. Hawkins ${ }^{80}$, T. Hayashi ${ }^{161}$, D. Hayden ${ }^{89}$, C.P. Hays ${ }^{119}$, H.S. Hayward ${ }^{73}$, S.J. Haywood ${ }^{130}$, S.J. Head ${ }^{18}$, T. Heck ${ }^{82}$, V. Hedberg ${ }^{80}$, L. Heelan ${ }^{8}$, S. Heim ${ }^{121}$, B. Heinemann ${ }^{15}$, S. Heisterkamp ${ }^{36}$, J. Hejbal ${ }^{126}$, L. Helary ${ }^{22}$, C. Heller ${ }^{99}$, M. Heller ${ }^{30}$, S. Hellman ${ }^{147 a, 147 b}$, D. Hellmich ${ }^{21}$, C. Helsens ${ }^{30}$, J. Henderson ${ }^{119}$, R.C.W. Henderson ${ }^{71}$, A. Henrichs ${ }^{177}$, A.M. Henriques Correia ${ }^{30}$, S. Henrot-Versille ${ }^{116}$, C. Hensel ${ }^{54}$, G.H. Herbert ${ }^{16}$, C.M. Hernandez ${ }^{8}$, Y. Hernández Jiménez ${ }^{168}$, R. Herrberg-Schubert ${ }^{16}$, G. Herten ${ }^{48}$, R. Hertenberger ${ }^{99}$, L. Hervas ${ }^{30}$, G.G. Hesketh ${ }^{77}$, N.P. Hessey ${ }^{106}$, R. Hickling ${ }^{75}$, E. Higón-Rodriguez ${ }^{168}$, J.C. Hill ${ }^{28}$, K.H. Hiller ${ }^{42}$, S. Hillert ${ }^{21}$, S.J. Hillier ${ }^{18}$, I. Hinchliffe ${ }^{15}$, E. Hines ${ }^{121}$, M. Hirose ${ }^{117}$, D. Hirschbuehl ${ }^{176}$, J. Hobbs ${ }^{149}$, N. Hod ${ }^{106}$, M.C. Hodgkinson ${ }^{140}$, P. Hodgson ${ }^{140}$, A. Hoecker ${ }^{30}$, M.R. Hoeferkamp ${ }^{104}$, J. Hoffman ${ }^{40}$, D. Hoffmann ${ }^{84}$, J.I. Hofmann ${ }^{58 a}$, M. Hohlfeld ${ }^{82}$, S.O. Holmgren ${ }^{147 a}$, T.M. Hong ${ }^{121}$,

L. Hooft van Huysduynen ${ }^{109}$, J-Y. Hostachy ${ }^{55}$, S. Hou ${ }^{152}$, A. Hoummada ${ }^{136 a}$, J. Howard ${ }^{119}$, J. Howarth ${ }^{83}$, M. Hrabovsky ${ }^{114}$, I. Hristova ${ }^{16}$, J. Hrivnac ${ }^{116}$, T. Hryn'ova ${ }^{5}$, P.J. Hsu ${ }^{82}$, S.-C. Hsu ${ }^{139}$, D. $\mathrm{Hu}^{35}, \mathrm{X} . \mathrm{Hu}^{25}$, Y. Huang ${ }^{146 c}$, Z. Hubacek ${ }^{30}$, F. Hubaut ${ }^{84}$, F. Huegging ${ }^{21}$,

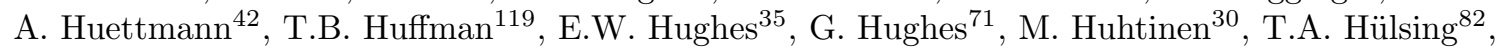
M. Hurwitz ${ }^{15}$, N. Huseynov ${ }^{64, c}$, J. Huston ${ }^{89}$, J. Huth ${ }^{57}$, G. Iacobucci ${ }^{49}$, G. Iakovidis ${ }^{10}$, I. Ibragimov $^{142}$, L. Iconomidou-Fayard ${ }^{116}$, J. Idarraga ${ }^{116}$, P. Iengo ${ }^{103 a}$, O. Igonkina ${ }^{106}$,

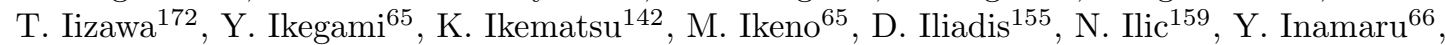
T. Ince ${ }^{100}$, P. Ioannou ${ }^{9}$, M. Iodice ${ }^{135 a}$, K. Iordanidou ${ }^{9}$, V. Ippolito ${ }^{133 a, 133 b}$, A. Irles Quiles ${ }^{168}$,

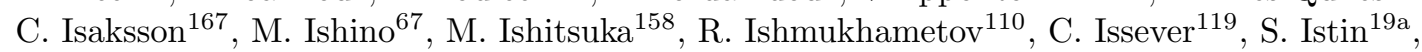
A.V. Ivashin ${ }^{129}$, W. Iwanski ${ }^{39}$, H. Iwasaki ${ }^{65}$, J.M. Izen ${ }^{41}$, V. Izzo ${ }^{103 a}$, B. Jackson ${ }^{121}$, 
J.N. Jackson ${ }^{73}$, M. Jackson ${ }^{73}$, P. Jackson ${ }^{1}$, M.R. Jaekel ${ }^{30}$, V. Jain ${ }^{2}$, K. Jakobs ${ }^{48}$, S. Jakobsen ${ }^{36}$, T. Jakoubek ${ }^{126}$, J. Jakubek ${ }^{127}$, D.O. Jamin ${ }^{152}$, D.K. Jana ${ }^{112}$, E. Jansen ${ }^{77}$, H. Jansen ${ }^{30}$, J. Janssen ${ }^{21}$, M. Janus ${ }^{171}$, R.C. Jared ${ }^{174}$, G. Jarlskog ${ }^{80}$, L. Jeanty ${ }^{57}$, G.-Y. Jeng ${ }^{151}$, I. Jen-La Plante ${ }^{31}$, D. Jennens ${ }^{87}$, P. Jenni ${ }^{48, t}$, J. Jentzsch ${ }^{43}$, C. Jeske ${ }^{171}$, S. Jézéquel ${ }^{5}$, M.K. Jha ${ }^{20 a}$, H. Ji ${ }^{174}$, W. Ji ${ }^{82}$, J. Jia ${ }^{149}$, Y. Jiang ${ }^{33 b}$, M. Jimenez Belenguer ${ }^{42}$, S. Jin ${ }^{33 a}$, A. Jinaru ${ }^{26 a}$, O. Jinnouchi ${ }^{158}$, M.D. Joergensen ${ }^{36}$, D. Joffe ${ }^{40}$, K.E. Johansson ${ }^{147 a}$, P. Johansson ${ }^{140}$, K.A. Johns ${ }^{7}$, K. Jon-And ${ }^{147 a, 147 b}$, G. Jones ${ }^{171}$, R.W.L. Jones ${ }^{71}$, T.J. Jones ${ }^{73}$, P.M. Jorge ${ }^{125 a}$, K.D. Joshi ${ }^{83}$, J. Jovicevic ${ }^{148}$, X. Ju ${ }^{174}$, C.A. Jung ${ }^{43}$, R.M. Jungst ${ }^{30}$, P. Jussel ${ }^{61}$, A. Juste Rozas ${ }^{12, r}$, M. Kaci ${ }^{168}$, A. Kaczmarska ${ }^{39}$, P. Kadlecik ${ }^{36}$, M. Kado ${ }^{116}$, H. Kagan ${ }^{110}$, M. Kagan ${ }^{144}$, E. Kajomovitz ${ }^{45}$, S. Kalinin ${ }^{176}$, S. Kama ${ }^{40}$, N. Kanaya ${ }^{156}$, M. Kaneda ${ }^{30}$, S. Kaneti ${ }^{28}$, T. Kanno ${ }^{158}$, V.A. Kantserov ${ }^{97}$, J. Kanzaki ${ }^{65}$, B. Kaplan ${ }^{109}$, A. Kapliy ${ }^{31}$, D. Kar ${ }^{53}$, K. Karakostas ${ }^{10}$, N. Karastathis ${ }^{10}$, M. Karnevskiy ${ }^{82}$, S.N. Karpov ${ }^{64}$, K. Karthik ${ }^{109}$, V. Kartvelishvili ${ }^{71}$, A.N. Karyukhin ${ }^{129}$, L. Kashif ${ }^{174}$, G. Kasieczka ${ }^{58 b}$, R.D. Kass ${ }^{110}$, A. Kastanas ${ }^{14}$, Y. Kataoka ${ }^{156}$, A. Katre ${ }^{49}$, J. Katzy ${ }^{42}$, V. Kaushik ${ }^{7}$, K. Kawagoe ${ }^{69}$, T. Kawamoto ${ }^{156}$, G. Kawamura ${ }^{54}$, S. Kazama ${ }^{156}$, V.F. Kazanin ${ }^{108}$, M.Y. Kazarinov ${ }^{64}$, R. Keeler ${ }^{170}$, P.T. Keener ${ }^{121}$, R. Kehoe ${ }^{40}$, M. Keil ${ }^{54}$, J.S. Keller ${ }^{139}$, H. Keoshkerian ${ }^{5}$, O. Kepka ${ }^{126}$, B.P. Kerševan ${ }^{74}$, S. Kersten ${ }^{176}$, K. Kessoku ${ }^{156}$, J. Keung ${ }^{159}$, F. Khalil-zada ${ }^{11}$, H. Khandanyan ${ }^{147 a, 147 b}$, A. Khanov ${ }^{113}$, D. Kharchenko ${ }^{64}$, A. Khodinov ${ }^{97}$, A. Khomich ${ }^{58 a}$, T.J. Khoo ${ }^{28}$, G. Khoriauli ${ }^{21}$, A. Khoroshilov ${ }^{176}$, V. Khovanskiy ${ }^{96}$, E. Khramov ${ }^{64}$, J. Khubua ${ }^{51 b}$, H. Kim ${ }^{147 a, 147 b}$, S.H. Kim ${ }^{161}$, N. Kimura ${ }^{172}$, O. Kind ${ }^{16}$, B.T. King ${ }^{73}$, M. King ${ }^{66}$, R.S.B. King ${ }^{119}$, S.B. King ${ }^{169}$, J. Kirk ${ }^{130}$, A.E. Kiryunin ${ }^{100}$, T. Kishimoto ${ }^{66}$, D. Kisielewska ${ }^{38 a}$, T. Kitamura ${ }^{66}$, T. Kittelmann ${ }^{124}$, K. Kiuchi ${ }^{161}$, E. Kladiva ${ }^{145 b}$, M. Klein ${ }^{73}$, U. Klein ${ }^{73}$, K. Kleinknecht ${ }^{82}$, P. Klimek ${ }^{147 a, 147 b}$, A. Klimentov ${ }^{25}$, R. Klingenberg ${ }^{43}$, J.A. Klinger ${ }^{83}$, E.B. Klinkby ${ }^{36}$, T. Klioutchnikova ${ }^{30}$, P.F. Klok ${ }^{105}$, E.-E. Kluge ${ }^{58 a}$, P. Kluit ${ }^{106}$, S. Kluth ${ }^{100}$, E. Kneringer ${ }^{61}$, E.B.F.G. Knoops ${ }^{84}$, A. Knue ${ }^{54}$, B.R. Ko ${ }^{45}$, T. Kobayashi ${ }^{156}$, M. Kobel ${ }^{44}$, M. Kocian ${ }^{144}$, P. Kodys ${ }^{128}$, S. Koenig ${ }^{82}$, P. Koevesarki ${ }^{21}$, T. Koffas ${ }^{29}$, E. Koffeman ${ }^{106}$, L.A. Kogan ${ }^{119}$, S. Kohlmann ${ }^{176}$, Z. Kohout ${ }^{127}$, T. Kohriki ${ }^{65}$, T. Koi ${ }^{144}$, H. Kolanoski ${ }^{16}$, I. Koletsou ${ }^{5}$, J. Koll ${ }^{89}$, A.A. Komar ${ }^{95, *}$, Y. Komori ${ }^{156}$, T. Kondo ${ }^{65}$, K. Köneke ${ }^{48}$, A.C. König ${ }^{105}$, T. Kono ${ }^{65, u}$, R. Konoplich ${ }^{109, v}$, N. Konstantinidis ${ }^{77}$, R. Kopeliansky ${ }^{153}$, S. Koperny ${ }^{38 a}$, L. Köpke ${ }^{82}$, A.K. Kopp ${ }^{48}$, K. Korcyl ${ }^{39}$, K. Kordas ${ }^{155}$, A. Korn ${ }^{46}$, A.A. Korol ${ }^{108}$, I. Korolkov ${ }^{12}$ E.V. Korolkova ${ }^{140}$, V.A. Korotkov ${ }^{129}$, O. Kortner ${ }^{100}$, S. Kortner ${ }^{100}$, V.V. Kostyukhin ${ }^{21}$, S. Kotov ${ }^{100}$, V.M. Kotov ${ }^{64}$, A. Kotwal ${ }^{45}$, C. Kourkoumelis ${ }^{9}$, V. Kouskoura ${ }^{155}$, A. Koutsman ${ }^{160 a}$, R. Kowalewski ${ }^{170}$, T.Z. Kowalski ${ }^{38 a}$, W. Kozanecki ${ }^{137}$, A.S. Kozhin ${ }^{129}$, V. Kral ${ }^{127}$, V.A. Kramarenko ${ }^{98}$, G. Kramberger ${ }^{74}$, M.W. Krasny ${ }^{79}$, A. Krasznahorkay ${ }^{109}$, J.K. Kraus ${ }^{21}$, A. Kravchenko ${ }^{25}$, S. Kreiss ${ }^{109}$, J. Kretzschmar ${ }^{73}$, K. Kreutzfeldt ${ }^{52}$, N. Krieger ${ }^{54}$, P. Krieger ${ }^{159}$, K. Kroeninger ${ }^{54}$, H. Kroha ${ }^{100}$, J. Kroll ${ }^{121}$, J. Kroseberg ${ }^{21}$, J. Krstic ${ }^{13 a}$, U. Kruchonak ${ }^{64}$, H. Krüger ${ }^{21}$, T. Kruker ${ }^{17}$, N. Krumnack ${ }^{63}$, Z.V. Krumshteyn ${ }^{64}$, A. Kruse ${ }^{174}$, M.C. Kruse ${ }^{45}$, M. Kruskal ${ }^{22}$, T. Kubota ${ }^{87}$, S. Kuday ${ }^{4 a}$, S. Kuehn ${ }^{48}$, A. Kugel ${ }^{58 c}$, T. Kuhl ${ }^{42}$, V. Kukhtin ${ }^{64}$, Y. Kulchitsky ${ }^{91}$, S. Kuleshov ${ }^{32 b}$, M. Kuna ${ }^{133 a, 133 b}$, J. Kunkle ${ }^{121}$, A. Kupco ${ }^{126}$, H. Kurashige ${ }^{66}$, M. Kurata ${ }^{161}$, Y.A. Kurochkin ${ }^{91}$, R. Kurumida ${ }^{66}$, V. Kus ${ }^{126}$, E.S. Kuwertz ${ }^{148}$, M. Kuze ${ }^{158}$, J. Kvita ${ }^{143}$, R. Kwee ${ }^{16}$, A. La Rosa ${ }^{49}$, L. La Rotonda ${ }^{37 a, 37 b}$, L. Labarga ${ }^{81}$, S. Lablak ${ }^{136 a}$, C. Lacasta $^{168}$, F. Lacava ${ }^{133 a, 133 b}$, J. Lacey ${ }^{29}$, H. Lacker ${ }^{16}$, D. Lacour ${ }^{79}$, V.R. Lacuesta ${ }^{168}$,

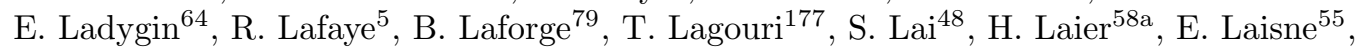
L. Lambourne ${ }^{77}$, C.L. Lampen ${ }^{7}$, W. Lampl ${ }^{7}$, E. Lançon ${ }^{137}$, U. Landgraf ${ }^{48}$, M.P.J. Landon ${ }^{75}$, V.S. Lang ${ }^{58 a}$, C. Lange ${ }^{42}$, A.J. Lankford ${ }^{164}$, F. Lanni ${ }^{25}$, K. Lantzsch $^{30}$, A. Lanza ${ }^{120 a}$, S. Laplace ${ }^{79}$, C. Lapoire ${ }^{21}$, J.F. Laporte ${ }^{137}$, T. Lari90a, A. Larner ${ }^{119}$, M. Lassnig ${ }^{30}$, P. Laurelli" ${ }^{47}$,

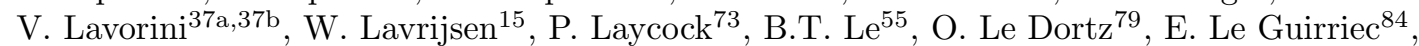
E. Le Menedeu ${ }^{12}$, T. LeCompte ${ }^{6}$, F. Ledroit-Guillon ${ }^{55}$, C.A. Lee ${ }^{152}$, H. Lee ${ }^{106}$, J.S.H. Lee ${ }^{117}$, S.C. Lee ${ }^{152}$, L. Lee ${ }^{177}$, G. Lefebvre ${ }^{79}$, M. Lefebvre ${ }^{170}$, M. Legendre ${ }^{137}$, F. Legger ${ }^{99}$, C. Leggett ${ }^{15}$, A. Lehan $^{73}$, M. Lehmacher ${ }^{21}$, G. Lehmann Miotto ${ }^{30}$, A.G. Leister ${ }^{177}$, M.A.L. Leite ${ }^{24 d}$, R. Leitner ${ }^{128}$, D. Lellouch ${ }^{173}$, B. Lemmer ${ }^{54}$, V. Lendermann ${ }^{58 a}$, K.J.C. Leney ${ }^{146 c}$, T. Lenz ${ }^{106}$,

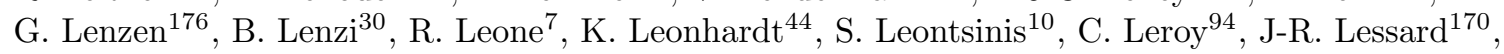


C.G. Lester $^{28}$, C.M. Lester ${ }^{121}$, J. Levêque ${ }^{5}$, D. Levin ${ }^{88}$, L.J. Levinson ${ }^{173}$, A. Lewis ${ }^{119}$, G.H. Lewis ${ }^{109}$, A.M. Leyko ${ }^{21}$, M. Leyton ${ }^{16}$, B. Li ${ }^{33 b, w}$, B. Li ${ }^{84}, \mathrm{H}^{2} \mathrm{Li}^{149}$, H.L. Li ${ }^{31}, \mathrm{~S}^{2} \mathrm{Li}^{45}$,

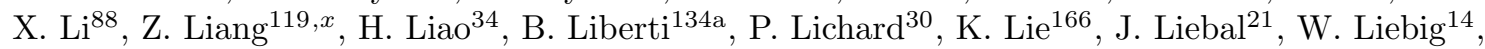
C. $\operatorname{Limbach}^{21}$, A. Limosani ${ }^{87}$, M. Limper ${ }^{62}$, S.C. $\operatorname{Lin}^{152, y}$, F. Linde ${ }^{106}$, B.E. Lindquist ${ }^{149}$, J.T. Linnemann ${ }^{89}$, E. Lipeles ${ }^{121}$, A. Lipniacka ${ }^{14}$, M. Lisovyi ${ }^{42}$, T.M. Liss ${ }^{166}$, D. Lissauer ${ }^{25}$, A. Lister ${ }^{169}$, A.M. Litke138, B. Liu ${ }^{152}$, D. Liu ${ }^{152}$, J.B. Liu ${ }^{33 b}$, K. Liu 33 b, $z$, L. Liu ${ }^{88}$, M. Liu ${ }^{45}$, M. Liu ${ }^{33 b}$, Y. Liu ${ }^{33 b}$, M. Livan ${ }^{120 a, 120 b}$, S.S.A. Livermore ${ }^{119}$, A. Lleres ${ }^{55}$, J. Llorente Merino ${ }^{81}$, S.L. Lloyd ${ }^{75}$, F. Lo Sterzo ${ }^{133 a, 133 b}$, E. Lobodzinska ${ }^{42}$, P. Loch $^{7}$, W.S. Lockman ${ }^{138}$,

T. Loddenkoetter ${ }^{21}$, F.K. Loebinger ${ }^{83}$, A.E. Loevschall-Jensen ${ }^{36}$, A. Loginov ${ }^{177}$, C.W. Loh ${ }^{169}$, T. Lohse ${ }^{16}$, K. Lohwasser ${ }^{48}$, M. Lokajicek ${ }^{126}$, V.P. Lombardo ${ }^{5}$, J.D. Long ${ }^{88}$, R.E. Long ${ }^{71}$, L. Lopes ${ }^{125 a}$, D. Lopez Mateos ${ }^{57}$, B. Lopez Paredes ${ }^{140}$, J. Lorenz ${ }^{99}$, N. Lorenzo Martinez ${ }^{116}$, M. Losada ${ }^{163}$, P. Loscutoff ${ }^{15}$, M.J. Losty ${ }^{160 a, *}$, X. Lou ${ }^{41}$, A. Lounis ${ }^{116}$, J. Love ${ }^{6}$, P.A. Love ${ }^{71}$, A.J. Lowe ${ }^{144, g}$, F. Lu ${ }^{33 a}$, H.J. Lubatti ${ }^{139}$, C. Luci ${ }^{133 a, 133 b}$, A. Lucotte ${ }^{55}$, D. Ludwig ${ }^{42}$,

I. Ludwig ${ }^{48}$, F. Luehring ${ }^{60}$, W. Lukas ${ }^{61}$, L. Luminari ${ }^{133 a}$, E. Lund ${ }^{118}$, J. Lundberg ${ }^{147 a, 147 b}$, O. Lundberg ${ }^{147 a, 147 b}$, B. Lund-Jensen ${ }^{148}$, M. Lungwitz ${ }^{82}$, D. Lynn ${ }^{25}$, R. Lysak ${ }^{126}$, E. Lytken ${ }^{80}$, H. $\mathrm{Ma}^{25}$, L.L. Ma ${ }^{33 \mathrm{~d}}$, G. Maccarrone ${ }^{47}$, A. Macchiolo ${ }^{100}$, B. Maček ${ }^{74}$, J. Machado Miguens ${ }^{125 a}$, D. Macina ${ }^{30}$, R. Mackeprang ${ }^{36}$, R. Madar ${ }^{48}$, R.J. Madaras ${ }^{15}$, H.J. Maddocks ${ }^{71}$, W.F. Mader ${ }^{44}$, A. Madsen ${ }^{167}$, M. Maeno ${ }^{8}$, T. Maeno ${ }^{25}$, L. Magnoni ${ }^{164}$, E. Magradze $^{54}$, K. Mahboubi ${ }^{48}$, J. Mahlstedt ${ }^{106}$, S. Mahmoud ${ }^{73}$, G. Mahout ${ }^{18}$, C. Maiani ${ }^{137}$, C. Maidantchik ${ }^{24 a}$, A. Maio ${ }^{125 a, d}$, S. Majewski ${ }^{115}$, Y. Makida ${ }^{65}$, N. Makovec ${ }^{116}$, P. Mal ${ }^{137, a a}$, B. Malaescu ${ }^{79}$, Pa. Malecki ${ }^{39}$, V.P. Maleev ${ }^{122}$, F. Malek ${ }^{55}$, U. Mallik ${ }^{62}$, D. Malon ${ }^{6}$, C. Malone ${ }^{144}$, S. Maltezos ${ }^{10}$, V.M. Malyshev ${ }^{108}$, S. Malyukov ${ }^{30}$, J. Mamuzic ${ }^{13 b}$, L. Mandelli ${ }^{90 a}$, I. Mandić ${ }^{74}$, R. Mandrysch ${ }^{62}$, J. Maneira ${ }^{125 a}$, A. Manfredini ${ }^{100}$, L. Manhaes de Andrade Filho ${ }^{24 b}$, J.A. Manjarres Ramos ${ }^{137}$, A. Mann ${ }^{99}$, P.M. Manning ${ }^{138}$, A. Manousakis-Katsikakis ${ }^{9}$, B. Mansoulie ${ }^{137}$, R. Mantifel ${ }^{86}$, L. Mapelli ${ }^{30}$, L. March ${ }^{168}$, J.F. Marchand ${ }^{29}$, F. Marchese ${ }^{134 a, 134 b}$, G. Marchiori ${ }^{79}$, M. Marcisovsky ${ }^{126}$, C.P. Marino ${ }^{170}$, C.N. Marques ${ }^{125 a}$, F. Marroquim ${ }^{24 a}$, Z. Marshall ${ }^{15}$,

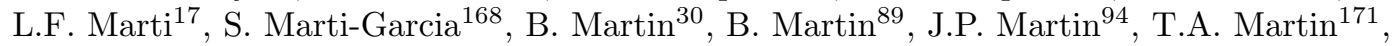
V.J. Martin ${ }^{46}$, B. Martin dit Latour ${ }^{49}$, H. Martinez ${ }^{137}$, M. Martinez ${ }^{12, r}$, S. Martin-Haugh ${ }^{150}$, A.C. Martyniuk ${ }^{170}$, M. Marx ${ }^{139}$, F. Marzano ${ }^{133 a}$, A. Marzin ${ }^{112}$, L. Masetti ${ }^{82}$, T. Mashimo ${ }^{156}$, R. Mashinistov ${ }^{95}$, J. Masik ${ }^{83}$, A.L. Maslennikov ${ }^{108}$, I. Massa ${ }^{20 a, 20 b}$, N. Massol ${ }^{5}$, P. Mastrandrea ${ }^{149}$, A. Mastroberardino ${ }^{37 a, 37 b}$, T. Masubuchi ${ }^{156}$, H. Matsunaga ${ }^{156}$, T. Matsushita ${ }^{66}$, P. Mättig ${ }^{176}$, S. Mättig ${ }^{42}$, J. Mattmann ${ }^{82}$, C. Mattravers ${ }^{119, e}$, J. Maurer ${ }^{84}$, S.J. Maxfield ${ }^{73}$, D.A. Maximov ${ }^{108, h}$, R. Mazini ${ }^{152}$, L. Mazzaferro ${ }^{134 a, 134 b}$, M. Mazzanti90a, G. Mc Goldrick ${ }^{159}$, S.P. Mc Kee ${ }^{88}$, A. McCarn ${ }^{88}$, R.L. McCarthy ${ }^{149}$, T.G. McCarthy ${ }^{29}$, N.A. McCubbin ${ }^{130}$, K.W. McFarlane ${ }^{56, *}$, J.A. Mcfayden ${ }^{140}$, G. Mchedlidze ${ }^{51 b}$, T. Mclaughlan ${ }^{18}$, S.J. McMahon ${ }^{130}$, R.A. McPherson ${ }^{170, k}$, A. Meade ${ }^{85}$, J. Mechnich ${ }^{106}$, M. Mechtel $^{176}$,

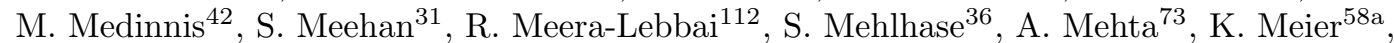
C. Meineck $^{99}$, B. Meirose ${ }^{80}$, C. Melachrinos ${ }^{31}$, B.R. Mellado Garcia ${ }^{146 c}$, F. Meloni ${ }^{90 a, 90 b}$, L. Mendoza Navas ${ }^{163}$, A. Mengarelli ${ }^{20 a, 20 b}$, S. Menke ${ }^{100}$, E. Meoni ${ }^{162}$, K.M. Mercurio ${ }^{57}$, S. Mergelmeyer ${ }^{21}$, N. Meric ${ }^{137}$, P. Mermod ${ }^{49}$, L. Merola103a,103b, C. Meroni ${ }^{90 a}$, F.S. Merritt ${ }^{31}$, H. Merritt ${ }^{110}$, A. Messina ${ }^{30, a b}$, J. Metcalfe ${ }^{25}$, A.S. Mete ${ }^{164}$, C. Meyer ${ }^{82}$, C. Meyer ${ }^{31}$, J-P. Meyer ${ }^{137}$, J. Meyer ${ }^{30}$, J. Meyer ${ }^{54}$, S. Michal ${ }^{30}$, R.P. Middleton ${ }^{130}$, S. Migas ${ }^{73}$, L. Mijović ${ }^{137}$, G. Mikenberg ${ }^{173}$, M. Mikestikova ${ }^{126}$, M. Mikuž ${ }^{74}$, D.W. Miller ${ }^{31}$, W.J. Mills ${ }^{169}$, C. Mills ${ }^{57}$, A. Milov $^{173}$, D.A. Milstead ${ }^{147 a, 147 b}$, D. Milstein ${ }^{173}$, A.A. Minaenko ${ }^{129}$, M. Miñano Moya ${ }^{168}$, I.A. Minashvili ${ }^{64}$, A.I. Mincer ${ }^{109}$, B. Mindur ${ }^{38 a}$, M. Mineev ${ }^{64}$, Y. Ming ${ }^{174}$, L.M. Mir ${ }^{12}$, G. Mirabelli ${ }^{133 a}$, T. Mitani ${ }^{172}$, J. Mitrevski ${ }^{138}$, V.A. Mitsou ${ }^{168}$, S. Mitsui ${ }^{65}$, P.S. Miyagawa ${ }^{140}$, J.U. Mjörnmark ${ }^{80}$, T. Moa ${ }^{147 a, 147 b}$, V. Moeller ${ }^{28}$, S. Mohapatra ${ }^{149}$, W. Mohr ${ }^{48}$, S. Molander ${ }^{147 a, 147 b}$, R. Moles-Valls ${ }^{168}$, A. Molfetas ${ }^{30}$, K. Mönig ${ }^{42}$, C. Monini ${ }^{55}$, J. Monk ${ }^{36}$, E. Monnier ${ }^{84}$, J. Montejo Berlingen ${ }^{12}$, F. Monticelli ${ }^{70}$, S. Monzani ${ }^{20 a, 20 b}$, R.W. Moore ${ }^{3}$, C. Mora Herrera ${ }^{49}$, A. Moraes ${ }^{53}$, N. Morange ${ }^{62}$, J. Morel ${ }^{54}$, D. Moreno ${ }^{82}$, M. Moreno Llácer ${ }^{168}$, P. Morettini ${ }^{50 a}$, M. Morgenstern ${ }^{44}$, M. Morii ${ }^{57}$, S. Moritz ${ }^{82}$, A.K. Morley ${ }^{148}$, G. Mornacchi ${ }^{30}$, J.D. Morris ${ }^{75}$, L. Morvaj ${ }^{102}$, H.G. Moser ${ }^{100}$, M. Mosidze ${ }^{51 b}$, J. Moss ${ }^{110}$, R. Mount ${ }^{144}$, 
E. Mountricha ${ }^{25}$, S.V. Mouraviev ${ }^{95, *}$, E.J.W. Moyse ${ }^{85}$, R.D. Mudd ${ }^{18}$, F. Mueller ${ }^{58 a}$, J. Mueller ${ }^{124}$, K. Mueller ${ }^{21}$, T. Mueller ${ }^{28}$, T. Mueller ${ }^{82}$, D. Muenstermann ${ }^{49}$, Y. Munwes ${ }^{154}$, J.A. Murillo Quijada ${ }^{18}$, W.J. Murray ${ }^{130}$, I. Mussche ${ }^{106}$, E. Musto ${ }^{153}$, A.G. Myagkov ${ }^{129, a c}$, M. Myska ${ }^{126}$, O. Nackenhorst ${ }^{54}$, J. Nadal ${ }^{12}$, K. Nagai ${ }^{61}$, R. Nagai ${ }^{158}$, Y. Nagai ${ }^{84}$, K. Nagano ${ }^{65}$, A. Nagarkar ${ }^{110}$, Y. Nagasaka ${ }^{59}$, M. Nagel ${ }^{100}$, A.M. Nairz ${ }^{30}$, Y. Nakahama ${ }^{30}$, K. Nakamura ${ }^{65}$, T. Nakamura ${ }^{156}$, I. Nakano ${ }^{111}$, H. Namasivayam ${ }^{41}$, G. Nanava ${ }^{21}$, A. Napier ${ }^{162}$, R. Narayan ${ }^{58 b}$ M. Nash ${ }^{77, e}$, T. Nattermann ${ }^{21}$, T. Naumann ${ }^{42}$, G. Navarro ${ }^{163}$, H.A. Neal ${ }^{88}$, P.Yu. Nechaeva ${ }^{95}$, T.J. Neep ${ }^{83}$, A. Negri ${ }^{120 a, 120 b}$, G. Negri ${ }^{30}$, M. Negrini ${ }^{20 a}$, S. Nektarijevic ${ }^{49}$, A. Nelson ${ }^{164}$, T.K. Nelson ${ }^{144}$, S. Nemecek ${ }^{126}$, P. Nemethy ${ }^{109}$, A.A. Nepomuceno ${ }^{24 a}$, M. Nessi ${ }^{30, a d}$, M.S. Neubauer ${ }^{166}$, M. Neumann ${ }^{176}$, A. Neusiedl ${ }^{82}$, R.M. Neves ${ }^{109}$, P. Nevski ${ }^{25}$, F.M. Newcomer ${ }^{121}$, P.R. Newman ${ }^{18}$, D.H. Nguyen ${ }^{6}$, V. Nguyen Thi Hong ${ }^{137}$, R.B. Nickerson ${ }^{119}$, R. Nicolaidou ${ }^{137}$, B. Nicquevert ${ }^{30}$, J. Nielsen ${ }^{138}$, N. Nikiforou ${ }^{35}$, A. Nikiforov ${ }^{16}$, V. Nikolaenko ${ }^{129, a c}$, I. Nikolic-Audit ${ }^{79}$, K. Nikolics ${ }^{49}$, K. Nikolopoulos ${ }^{18}$, P. Nilsson ${ }^{8}$, Y. Ninomiya ${ }^{156}$, A. Nisati ${ }^{133 a}$, R. Nisius ${ }^{100}$, T. Nobe ${ }^{158}$, L. Nodulman $^{6}$, M. Nomachi ${ }^{117}$, I. Nomidis ${ }^{155}$, S. Norberg ${ }^{112}$, M. Nordberg ${ }^{30}$, J. Novakova ${ }^{128}$, M. Nozaki ${ }^{65}$, L. Nozka ${ }^{114}$, K. Ntekas ${ }^{10}$, A.-E. Nuncio-Quiroz ${ }^{21}$, G. Nunes Hanninger ${ }^{87}$, T. Nunnemann ${ }^{99}$, E. Nurse ${ }^{77}$, B.J. O'Brien ${ }^{46}$, F. O'grady ${ }^{7}$, D.C. O'Neil ${ }^{143}$, V. O'Shea ${ }^{53}$, L.B. Oakes ${ }^{99}$, F.G. Oakham ${ }^{29, f}$, H. Oberlack ${ }^{100}$, J. Ocariz ${ }^{79}$, A. Ochi ${ }^{66}$, M.I. Ochoa ${ }^{77}$, S. Oda ${ }^{69}$, S. Odaka ${ }^{65}$, H. Ogren ${ }^{60}$, A. $\mathrm{Oh}^{83}$, S.H. $\mathrm{Oh}^{45}$, C.C. $\mathrm{Ohm}^{30}$, T. Ohshima ${ }^{102}$, W. Okamura ${ }^{117}$, H. Okawa ${ }^{25}$, Y. Okumura ${ }^{31}$,

T. Okuyama ${ }^{156}$, A. Olariu ${ }^{26 a}$, A.G. Olchevski ${ }^{64}$, S.A. Olivares Pino ${ }^{46}$, M. Oliveira ${ }^{125 a, i}$,

D. Oliveira Damazio ${ }^{25}$, E. Oliver Garcia ${ }^{168}$, D. Olivito ${ }^{121}$, A. Olszewski ${ }^{39}$, J. Olszowska ${ }^{39}$,

A. Onofre ${ }^{125 a, a e}$, P.U.E. Onyisi ${ }^{31, a f}$, C.J. Oram ${ }^{160 a}$, M.J. Oreglia ${ }^{31}$, Y. Oren ${ }^{154}$,

D. Orestano ${ }^{135 a, 135 b}$, N. Orlando ${ }^{72 a, 72 b}$, C. Oropeza Barrera ${ }^{53}$, R.S. Orr ${ }^{159}$, B. Osculati ${ }^{50 a, 50 b}$, R. Ospanov ${ }^{121}$, G. Otero y Garzon ${ }^{27}$, H. Otono ${ }^{69}$, J.P. Ottersbach ${ }^{106}$, M. Ouchrif ${ }^{136 d}$, E.A. Ouellette ${ }^{170}$, F. Ould-Saada ${ }^{118}$, A. Ouraou ${ }^{137}$, K.P. Oussoren ${ }^{106}$, Q. Ouyang ${ }^{33 a}$,

A. Ovcharova ${ }^{15}$, M. Owen ${ }^{83}$, S. Owen ${ }^{140}$, V.E. Ozcan ${ }^{19 a}$, N. Ozturk ${ }^{8}$, K. Pachal ${ }^{119}$,

A. Pacheco Pages ${ }^{12}$, C. Padilla Aranda ${ }^{12}$, S. Pagan Griso ${ }^{15}$, E. Paganis ${ }^{140}$, C. Pahl ${ }^{100}$, F. Paige ${ }^{25}$, P. Pais ${ }^{85}$, K. Pajchel ${ }^{118}$, G. Palacino ${ }^{160 b}$, S. Palestini ${ }^{30}$, D. Pallin ${ }^{34}$, A. Palma ${ }^{125 a}$, J.D. Palmer ${ }^{18}$, Y.B. $\operatorname{Pan}^{174}$, E. Panagiotopoulou ${ }^{10}$, J.G. Panduro Vazquez ${ }^{76}$, P. Pani ${ }^{106}$, N. Panikashvili ${ }^{88}$, S. Panitkin ${ }^{25}$, D. Pantea ${ }^{26 a}$, Th.D. Papadopoulou ${ }^{10}$, K. Papageorgiou ${ }^{155, q}$, A. Paramonov ${ }^{6}$, D. Paredes Hernandez ${ }^{34}$, M.A. Parker ${ }^{28}$, F. Parodi ${ }^{50 a, 50 b}$, J.A. Parsons ${ }^{35}$, U. Parzefall ${ }^{48}$, S. Pashapour ${ }^{54}$, E. Pasqualucci ${ }^{133 a}$, S. Passaggio ${ }^{50 a}$, A. Passeri ${ }^{135 a}$, F. Pastore ${ }^{135 a, 135 b, *}$, Fr. Pastore ${ }^{76}$, G. Pásztor ${ }^{49, a g}$, S. Pataraia ${ }^{176}$, N.D. Patel ${ }^{151}$, J.R. Pater ${ }^{83}$, S. Patricelli ${ }^{103 a, 103 b}$, T. Pauly ${ }^{30}$, J. Pearce ${ }^{170}$, M. Pedersen ${ }^{118}$, S. Pedraza Lopez ${ }^{168}$, M.I. Pedraza Morales ${ }^{174}$, S.V. Peleganchuk ${ }^{108}$, D. Pelikan ${ }^{167}$, H. Peng ${ }^{33 b}$, B. Penning ${ }^{31}$, A. Penson ${ }^{35}$, J. Penwell ${ }^{60}$, D.V. Perepelitsa ${ }^{35}$, T. Perez Cavalcanti ${ }^{42}$, E. Perez Codina ${ }^{160 a}$, M.T. Pérez García-Estañ ${ }^{168}$, V. Perez Reale ${ }^{35}$, L. Perini ${ }^{90 a, 90 b}$, H. Pernegger ${ }^{30}$, R. Perrino ${ }^{72 a}$, V.D. Peshekhonov ${ }^{64}$, K. Peters ${ }^{30}$, R.F.Y. Peters ${ }^{54, a h}$, B.A. Petersen ${ }^{30}$, J. Petersen ${ }^{30}$, T.C. Petersen ${ }^{36}$, E. Petit ${ }^{5}$, A. Petridis ${ }^{147 a, 147 b}$, C. Petridou ${ }^{155}$, E. Petrolo133a, F. Petrucci ${ }^{135 a, 135 b}$, M. Petteni ${ }^{143}$, R. Pezoa ${ }^{32 b}$, P.W. Phillips ${ }^{130}$, G. Piacquadio ${ }^{144}$, E. Pianori ${ }^{171}$, A. Picazio ${ }^{49}$, E. Piccaro ${ }^{75}$, M. Piccinini ${ }^{20 a}, 20 b$, S.M. Piec ${ }^{42}$, R. Piegaia ${ }^{27}$, D.T. Pignotti ${ }^{110}$, J.E. Pilcher ${ }^{31}$, A.D. Pilkington ${ }^{77}$, J. Pina ${ }^{125 a, d}$, M. Pinamonti ${ }^{165 a, 165 c, a i}$, A. Pinder ${ }^{119}$, J.L. Pinfold ${ }^{3}$, A. Pingel ${ }^{36}$, B. Pinto ${ }^{125 a}$, C. Pizio ${ }^{90 a, 90 b}$, M.-A. Pleier ${ }^{25}$, V. Pleskot ${ }^{128}$, E. Plotnikova ${ }^{64}$, P. Plucinski ${ }^{147 a, 147 b}$, S. Poddar $^{58 a}$, F. Podlyski ${ }^{34}$, R. Poettgen ${ }^{82}$, L. Poggioli ${ }^{116}$, D. Pohl $^{21}$, M. Pohl ${ }^{49}$, G. Polesello ${ }^{120 a}$, A. Policicchio ${ }^{37 a, 37 b}$, R. Polifka ${ }^{159}$, A. Polini ${ }^{20 a}$, C.S. Pollard ${ }^{45}$, V. Polychronakos ${ }^{25}$, D. Pomeroy $^{23}$, K. Pommès ${ }^{30}$, L. Pontecorvo ${ }^{133 a}$, B.G. Pope ${ }^{89}$, G.A. Popeneciu ${ }^{26 b}$, D.S. Popovic ${ }^{13 a}$, A. Poppleton ${ }^{30}$, X. Portell Bueso ${ }^{12}$, G.E. Pospelov ${ }^{100}$, S. Pospisil ${ }^{127}$, K. Potamianos $^{15}$, I.N. Potrap ${ }^{64}$, C.J. Potter ${ }^{150}$, C.T. Potter ${ }^{115}$, G. Poulard ${ }^{30}$, J. Poveda ${ }^{60}$,

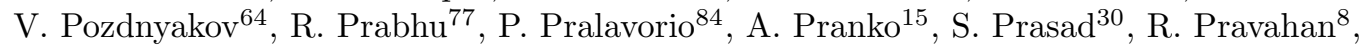
S. Prell ${ }^{63}$, D. Price ${ }^{83}$, J. Price ${ }^{73}$, L.E. Price ${ }^{6}$, D. Prieur ${ }^{124}$, M. Primavera ${ }^{72 a}$, M. Proissl ${ }^{46}$, K. Prokofiev ${ }^{109}$, F. Prokoshin ${ }^{32 b}$, E. Protopapadaki ${ }^{137}$, S. Protopopescu ${ }^{25}$, J. Proudfoot ${ }^{6}$, X. Prudent ${ }^{44}$, M. Przybycien ${ }^{38 a}$, H. Przysiezniak ${ }^{5}$, S. Psoroulas ${ }^{21}$, E. Ptacek ${ }^{115}$, E. Pueschel ${ }^{85}$, 
D. Puldon ${ }^{149}$, M. Purohit ${ }^{25, a j}$, P. Puzo ${ }^{116}$, Y. Pylypchenko ${ }^{62}$, J. Qian ${ }^{88}$, A. Quadt ${ }^{54}$, D.R. Quarrie ${ }^{15}$, W.B. Quayle ${ }^{146 c}$, D. Quilty ${ }^{53}$, V. Radeka ${ }^{25}$, V. Radescu ${ }^{42}$, P. Radloff ${ }^{115}$, F. Ragusa ${ }^{90 a, 90 b}$, G. Rahal ${ }^{179}$, S. Rajagopalan ${ }^{25}$, M. Rammensee ${ }^{48}$, M. Rammes ${ }^{142}$, A.S. Randle-Conde ${ }^{40}$, C. Rangel-Smith ${ }^{79}$, K. Rao ${ }^{164}$, F. Rauscher ${ }^{99}$, T.C. Rave ${ }^{48}$, T. Ravenscroft ${ }^{53}$, M. Raymond ${ }^{30}$, A.L. Read ${ }^{118}$, D.M. Rebuzzi ${ }^{120 a, 120 b}$, A. Redelbach ${ }^{175}$, G. Redlinger ${ }^{25}$, R. Reece ${ }^{121}$, K. Reeves ${ }^{41}$, A. Reinsch ${ }^{115}$, I. Reisinger ${ }^{43}$, M. Relich ${ }^{164}$, C. Rembser $^{30}$, Z.L. Ren ${ }^{152}$, A. Renaud ${ }^{116}$, M. Rescigno ${ }^{133 a}$, S. Resconi ${ }^{90 a}$, B. Resende ${ }^{137}$, P. Reznicek ${ }^{99}$, R. Rezvani ${ }^{94}$, R. Richter ${ }^{100}$, M. Ridel $^{79}$, P. Rieck ${ }^{16}$, M. Rijssenbeek ${ }^{149}$,

A. Rimoldi ${ }^{120 a, 120 b}$, L. Rinaldi ${ }^{20 a}$, R.R. Rios ${ }^{40}$, E. Ritsch ${ }^{61}$, I. Riu ${ }^{12}$, G. Rivoltella ${ }^{90 a, 90 b}$, F. Rizatdinova ${ }^{113}$, E. Rizvi ${ }^{75}$, S.H. Robertson ${ }^{86, k}$, A. Robichaud-Veronneau ${ }^{119}$, D. Robinson ${ }^{28}$, J.E.M. Robinson ${ }^{83}$, A. Robson ${ }^{53}$, J.G. Rocha de Lima ${ }^{107}$, C. Roda ${ }^{123 a, 123 b}$,

D. Roda Dos Santos ${ }^{126}$, L. Rodrigues ${ }^{30}$, A. Roe ${ }^{54}$, S. Roe ${ }^{30}$, O. Røhne ${ }^{118}$, S. Rolli ${ }^{162}$,

A. Romaniouk ${ }^{97}$, M. Romano ${ }^{20 a, 20 b}$, G. Romeo ${ }^{27}$, E. Romero Adam ${ }^{168}$, N. Rompotis ${ }^{139}$,

L. $\operatorname{Roos}^{79}$, E. $\operatorname{Ros}^{168}$, S. Rosati ${ }^{133 a}$, K. Rosbach ${ }^{49}$, A. Rose ${ }^{150}$, M. Rose ${ }^{76}$, P.L. Rosendahl ${ }^{14}$,

O. Rosenthal ${ }^{142}$, V. Rossetti ${ }^{12}$, E. Rossi ${ }^{103 a, 103 b}$, L.P. Rossi ${ }^{50 a}$, R. Rosten ${ }^{139}$, M. Rotaru ${ }^{26 a}$, I. Roth ${ }^{173}$, J. Rothberg ${ }^{139}$, D. Rousseau ${ }^{116}$, C.R. Royon ${ }^{137}$, A. Rozanov ${ }^{84}$, Y. Rozen ${ }^{153}$, X. Ruan ${ }^{146 c}$, F. Rubbo ${ }^{12}$, I. Rubinskiy ${ }^{42}$, V.I. Rud ${ }^{98}$, C. Rudolph ${ }^{44}$, M.S. Rudolph ${ }^{159}$, F. Rühr ${ }^{7}$, A. Ruiz-Martinez ${ }^{63}$, L. Rumyantsev ${ }^{64}$, Z. Rurikova ${ }^{48}$, N.A. Rusakovich ${ }^{64}$, A. Ruschke ${ }^{99}$, J.P. Rutherfoord ${ }^{7}$, N. Ruthmann ${ }^{48}$, P. Ruzicka ${ }^{126}$, Y.F. Ryabov ${ }^{122}$, M. Rybar ${ }^{128}$, G. Rybkin ${ }^{116}$, N.C. Ryder ${ }^{119}$, A.F. Saavedra ${ }^{151}$, A. Saddique ${ }^{3}$, I. Sadeh ${ }^{154}$, H.F-W. Sadrozinski ${ }^{138}$, R. Sadykov ${ }^{64}$, F. Safai Tehrani ${ }^{133 a}$, H. Sakamoto ${ }^{156}$, Y. Sakurai ${ }^{172}$, G. Salamanna ${ }^{75}$, A. Salamon ${ }^{134 a}$, M. Saleem ${ }^{112}$, D. Salek ${ }^{106}$, D. Salihagic ${ }^{100}$, A. Salnikov ${ }^{144}$, J. Salt ${ }^{168}$, B.M. Salvachua Ferrando ${ }^{6}$, D. Salvatore ${ }^{37 a, 37 b}$, F. Salvatore ${ }^{150}$, A. Salvucci ${ }^{105}$, A. Salzburger ${ }^{30}$, D. Sampsonidis ${ }^{155}$, A. Sanchez ${ }^{103 a, 103 b}$, J. Sánchez ${ }^{168}$, V. Sanchez Martinez ${ }^{168}$, H. Sandaker ${ }^{14}$, H.G. Sander ${ }^{82}$, M.P. Sanders ${ }^{99}$, M. Sandhoff ${ }^{176}$, T. Sandoval ${ }^{28}$, C. Sandoval ${ }^{163}$, R. Sandstroem ${ }^{100}$, D.P.C. Sankey ${ }^{130}$, A. Sansoni ${ }^{47}$, C. Santoni ${ }^{34}$, R. Santonico ${ }^{134 a, 134 b}$, H. Santos ${ }^{125 a}$,

I. Santoyo Castillo ${ }^{150}$, K. Sapp ${ }^{124}$, A. Sapronov ${ }^{64}$, J.G. Saraiva ${ }^{125 a}$, E. Sarkisyan-Grinbaum ${ }^{8}$, B. Sarrazin ${ }^{21}$, G. Sartisohn ${ }^{176}$, O. Sasaki ${ }^{65}$, Y. Sasaki ${ }^{156}$, N. Sasao ${ }^{67}$, I. Satsounkevitch ${ }^{91}$, G. Sauvage ${ }^{5, *}$, E. Sauvan ${ }^{5}$, J.B. Sauvan ${ }^{116}$, P. Savard ${ }^{159, f}$, V. Savinov ${ }^{124}$, D.O. Savu ${ }^{30}$, C. Sawyer ${ }^{119}$, L. Sawyer ${ }^{78, m}$, D.H. Saxon ${ }^{53}$, J. Saxon ${ }^{121}$, C. Sbarra ${ }^{20 a}$, A. Sbrizzi ${ }^{3}$, T. Scanlon ${ }^{30}$, D.A. Scannicchio ${ }^{164}$, M. Scarcella ${ }^{151}$, J. Schaarschmidt ${ }^{116}$, P. Schacht ${ }^{100}$, D. Schaefer ${ }^{121}$,

A. Schaelicke ${ }^{46}$, S. Schaepe ${ }^{21}$, S. Schaetzel ${ }^{58 b}$, U. Schäfer ${ }^{82}$, A.C. Schaffer ${ }^{116}$, D. Schaile ${ }^{99}$, R.D. Schamberger ${ }^{149}$, V. Scharf ${ }^{58 a}$, V.A. Schegelsky ${ }^{122}$, D. Scheirich ${ }^{88}$, M. Schernau ${ }^{164}$, M.I. Scherzer ${ }^{35}$, C. Schiavi ${ }^{50 a, 50 b}$, J. Schieck ${ }^{99}$, C. Schillo ${ }^{48}$, M. Schioppa ${ }^{37 a, 37 b}$, S. Schlenker ${ }^{30}$, E. Schmidt ${ }^{48}$, K. Schmieden ${ }^{30}$, C. Schmitt ${ }^{82}$, C. Schmitt ${ }^{99}$, S. Schmitt ${ }^{58 b}$, B. Schneider ${ }^{17}$, Y.J. Schnellbach ${ }^{73}$, U. Schnoor ${ }^{44}$, L. Schoeffel ${ }^{137}$, A. Schoening ${ }^{58 b}$, B.D. Schoenrock ${ }^{89}$, A.L.S. Schorlemmer ${ }^{54}$, M. Schott ${ }^{82}$, D. Schouten ${ }^{160 a}$, J. Schovancova ${ }^{25}$, M. Schram ${ }^{86}$, S. Schramm ${ }^{159}$, M. Schreyer ${ }^{175}$, C. Schroeder ${ }^{82}$, N. Schroer ${ }^{58 c}$, N. Schuh ${ }^{82}$, M.J. Schultens ${ }^{21}$, H.-C. Schultz-Coulon ${ }^{58 a}$, H. Schulz ${ }^{16}$, M. Schumacher ${ }^{48}$, B.A. Schumm ${ }^{138}$, Ph. Schune ${ }^{137}$, A. Schwartzman ${ }^{144}$, Ph. Schwegler ${ }^{100}$, Ph. Schwemling ${ }^{137}$, R. Schwienhorst ${ }^{89}$, J. Schwindling ${ }^{137}$, T. Schwindt ${ }^{21}$, M. Schwoerer ${ }^{5}$, F.G. Sciacca ${ }^{17}$, E. Scifo ${ }^{116}$, G. Sciolla ${ }^{23}$, W.G. Scott ${ }^{130}$, F. Scutti ${ }^{21}$, J. Searcy ${ }^{88}$, G. Sedor ${ }^{42}$, E. Sedykh ${ }^{122}$, S.C. Seidel ${ }^{104}$, A. Seiden ${ }^{138}$, F. Seifert ${ }^{44}$, J.M. Seixas ${ }^{24 a}$, G. Sekhniaidze ${ }^{103 a}$, S.J. Sekula ${ }^{40}$, K.E. Selbach ${ }^{46}$, D.M. Seliverstov ${ }^{122}$, G. Sellers ${ }^{73}$, M. Seman ${ }^{145 b}$, N. Semprini-Cesariin ${ }^{20 a, 20 b}$, C. Serfon ${ }^{30}$, L. Serin ${ }^{116}$, L. Serkin ${ }^{54}$, T. Serre ${ }^{84}$, R. Seuster ${ }^{160 a}$, H. Severini ${ }^{112}$, F. Sforza ${ }^{100}$, A. Sfyrla ${ }^{30}$, E. Shabalina ${ }^{54}$, M. Shamim ${ }^{115}$, L.Y. Shan ${ }^{33 a}$, J.T. Shank ${ }^{22}$, Q.T. Shao ${ }^{87}$, M. Shapiro ${ }^{15}$, P.B. Shatalov ${ }^{96}$, K. Shaw ${ }^{165 a, 165 c}$, P. Sherwood ${ }^{77}$, S. Shimizu ${ }^{66}$, M. Shimojima ${ }^{101}$, T. Shin ${ }^{56}$, M. Shiyakova ${ }^{64}$, A. Shmeleva ${ }^{95}$, M.J. Shochet ${ }^{31}$, D. Short ${ }^{119}$, S. Shrestha ${ }^{63}$, E. Shulga ${ }^{97}$, M.A. Shupe ${ }^{7}$, S. Shushkevich ${ }^{42}$, P. Sicho ${ }^{126}$, D. Sidorov ${ }^{113}$, A. Sidoti ${ }^{133 a}$, F. Siegert ${ }^{48}$, Dj. Sijacki ${ }^{13 a}$, O. Silbert ${ }^{173}$, J. Silva ${ }^{125 a}$, Y. Silver ${ }^{154}$, D. Silverstein ${ }^{144}$, S.B. Silverstein ${ }^{147 a}$, V. Simak ${ }^{127}$, O. Simard ${ }^{5}$, Lj. Simic ${ }^{13 a}$, S. Simion ${ }^{116}$, E. Simioni ${ }^{82}$, B. Simmons ${ }^{77}$, R. Simoniello ${ }^{90 a}$,90b, M. Simonyan ${ }^{36}$, P. Sinervo $^{159}$, N.B. Sinev ${ }^{115}$, V. Sipica ${ }^{142}$, G. Siragusa ${ }^{175}$, A. Sircar ${ }^{78}$, A.N. Sisakyan ${ }^{64, *}$, S.Yu. Sivoklokov ${ }^{98}$, 
J. Sjölin ${ }^{147 a, 147 b}$, T.B. Sjursen ${ }^{14}$, L.A. Skinnari ${ }^{15}$, H.P. Skottowe ${ }^{57}$, K.Yu. Skovpen ${ }^{108}$, P. Skubic ${ }^{112}$, M. Slater ${ }^{18}$, T. Slavicek ${ }^{127}$, K. Sliwa ${ }^{162}$, V. Smakhtin ${ }^{173}$, B.H. Smart ${ }^{46}$,

L. Smestad ${ }^{118}$, S.Yu. Smirnov ${ }^{97}$, Y. Smirnov ${ }^{97}$, L.N. Smirnova ${ }^{98, a k}$, O. Smirnova ${ }^{80}$, K.M. Smith ${ }^{53}$, M. Smizanska ${ }^{71}$, K. Smolek ${ }^{127}$, A.A. Snesarev ${ }^{95}$, G. Snidero ${ }^{75}$, J. Snow ${ }^{112}$, S. Snyder ${ }^{25}$,

R. Sobie ${ }^{170, k}$, F. Socher ${ }^{44}$, J. Sodomka ${ }^{127}$, A. Soffer ${ }^{154}$, D.A. Soh ${ }^{152, x}$, C.A. Solans ${ }^{30}$, M. Solar ${ }^{127}$, J. Solc ${ }^{127}$, E.Yu. Soldatov ${ }^{97}$, U. Soldevila ${ }^{168}$, E. Solfaroli Camillocci ${ }^{133 a, 133 b}$, A.A. Solodkov ${ }^{129}$, O.V. Solovyanov ${ }^{129}$, V. Solovyev ${ }^{122}$, N. Soni ${ }^{1}$, A. Sood ${ }^{15}$, V. Sopko ${ }^{127}$, B. Sopko ${ }^{127}$, M. Sosebee ${ }^{8}$, R. Soualah ${ }^{165 a, 165 c}$, P. Soueid ${ }^{94}$, A.M. Soukharev ${ }^{108}$, D. South ${ }^{42}$, S. Spagnolo ${ }^{72 a, 72 b}$, F. Spanò ${ }^{76}$, W.R. Spearman ${ }^{57}$, R. Spighi ${ }^{20 a}$, G. Spigo ${ }^{30}$, M. Spousta ${ }^{128}$, T. Spreitzer ${ }^{159}$, B. Spurlock ${ }^{8}$, R.D. St. Denis ${ }^{53}$, J. Stahlman ${ }^{121}$, R. Stamen ${ }^{58 a}$, E. Stanecka ${ }^{39}$, R.W. Stanek ${ }^{6}$, C. Stanescu ${ }^{135 a}$, M. Stanescu-Bellu ${ }^{42}$, M.M. Stanitzki ${ }^{42}$, S. Stapnes ${ }^{118}$, E.A. Starchenko ${ }^{129}$, J. Stark ${ }^{55}$, P. Staroba ${ }^{126}$, P. Starovoitov ${ }^{42}$, R. Staszewski ${ }^{39}$, P. Stavina ${ }^{145 a, *}$, G. Steele ${ }^{53}$, P. Steinbach ${ }^{44}$, P. Steinberg ${ }^{25}$, I. Stekl ${ }^{127}$, B. Stelzer ${ }^{143}$, H.J. Stelzer ${ }^{89}$, O. Stelzer-Chilton ${ }^{160 a}$, H. Stenzel ${ }^{52}$, S. Stern ${ }^{100}$, G.A. Stewart ${ }^{30}$, J.A. Stillings ${ }^{21}$, M.C. Stockton ${ }^{86}$, M. Stoebe ${ }^{86}$, K. Stoerig ${ }^{48}$, G. Stoicea ${ }^{26 a}$, S. Stonjek ${ }^{100}$, A.R. Stradling 8 , A. Straessner ${ }^{44}$, J. Strandberg ${ }^{148}$,

S. Strandberg ${ }^{147 a, 147 b}$, A. Strandlie ${ }^{118}$, E. Strauss ${ }^{144}$, M. Strauss ${ }^{112}$, P. Strizenec ${ }^{145 b}$, R. Ströhmer ${ }^{175}$, D.M. Strom ${ }^{115}$, R. Stroynowski ${ }^{40}$, S.A. Stucci ${ }^{17}$, B. Stugu ${ }^{14}$, I. Stumer ${ }^{25, *}$, J. Stupak ${ }^{149}$, P. Sturm ${ }^{176}$, N.A. Styles ${ }^{42}$, D. Su ${ }^{144}$, HS. Subramania ${ }^{3}$, R. Subramaniam ${ }^{78}$, A. Succurro ${ }^{12}$, Y. Sugaya ${ }^{117}$, C. Suhr ${ }^{107}$, M. Suk ${ }^{127}$, V.V. Sulin ${ }^{95}$, S. Sultansoy ${ }^{4 c}$, T. Sumida ${ }^{67}$, X. $\operatorname{Sun}^{55}$, J.E. Sundermann ${ }^{48}$, K. Suruliz ${ }^{140}$, G. Susinno ${ }^{37 a, 37 b}$, M.R. Sutton ${ }^{150}$, Y. Suzuki ${ }^{65}$, M. Svatos ${ }^{126}$, S. Swedish ${ }^{169}$, M. Swiatlowski ${ }^{144}$, I. Sykora ${ }^{145 a}$, T. Sykora ${ }^{128}$, D. Ta ${ }^{89}$, K. Tackmann ${ }^{42}$, J. Taenzer ${ }^{159}$, A. Taffard ${ }^{164}$, R. Tafirout ${ }^{160 a}$, N. Taiblum ${ }^{154}$, Y. Takahashi ${ }^{102}$,

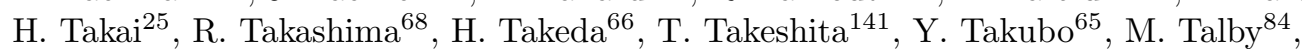
A.A. Talyshev ${ }^{108, h}$, J.Y.C. Tam ${ }^{175}$, M.C. Tamsett ${ }^{78, a l}$, K.G. Tan ${ }^{87}$, J. Tanaka ${ }^{156}$, R. Tanaka ${ }^{116}$, S. Tanaka ${ }^{132}$, S. Tanaka ${ }^{65}$, A.J. Tanasijczuk ${ }^{143}$, K. Tani ${ }^{66}$, N. Tannoury ${ }^{84}$, S. Tapprogge ${ }^{82}$,

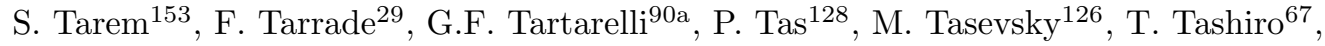
E. Tassi ${ }^{37 a, 37 b}$, A. Tavares Delgado ${ }^{125 a}$, Y. Tayalati ${ }^{136 d}$, C. Taylor ${ }^{77}$, F.E. Taylor ${ }^{93}$, G.N. Taylor ${ }^{87}$, W. Taylor ${ }^{160 b}$, F.A. Teischinger ${ }^{30}$, M. Teixeira Dias Castanheira ${ }^{75}$, P. Teixeira-Dias ${ }^{76}$, K.K. Temming ${ }^{48}$, H. Ten Kate ${ }^{30}$, P.K. Teng ${ }^{152}$, S. Terada ${ }^{65}$, K. Terashi ${ }^{156}$, J. Terron ${ }^{81}$, S. Terzo ${ }^{100}$, M. Testa ${ }^{47}$, R.J. Teuscher ${ }^{159, k}$, J. Therhaag ${ }^{21}$, T. Theveneaux-Pelzer ${ }^{34}$, S. Thoma ${ }^{48}$, J.P. Thomas ${ }^{18}$, E.N. Thompson ${ }^{35}$, P.D. Thompson ${ }^{18}$, P.D. Thompson ${ }^{159}$, A.S. Thompson ${ }^{53}$, L.A. Thomsen ${ }^{36}$, E. Thomson ${ }^{121}$, M. Thomson ${ }^{28}$, W.M. Thong ${ }^{87}$, R.P. Thun ${ }^{88, *}$, F. $\operatorname{Tian}^{35}$, M.J. Tibbetts ${ }^{15}$, T. Tic ${ }^{126}$, V.O. Tikhomirov ${ }^{95, a m}$, Yu.A. Tikhonov ${ }^{108, h}$, S. Timoshenko ${ }^{97}$, E. Tiouchichine ${ }^{84}$, P. Tipton ${ }^{177}$, S. Tisserant ${ }^{84}$, T. Todorov ${ }^{5}$, S. Todorova-Nova ${ }^{128}$, B. Toggerson ${ }^{164}$, J. Tojo ${ }^{69}$, S. Tokár ${ }^{145 a}$, K. Tokushuku ${ }^{65}$, K. Tollefson ${ }^{89}$, L. Tomlinson ${ }^{83}$, M. Tomoto ${ }^{102}$, L. Tompkins ${ }^{31}$, K. Toms ${ }^{104}$, A. Tonoyan ${ }^{14}$, N.D. Topilin ${ }^{64}$, E. Torrence ${ }^{115}$, H. Torres ${ }^{143}$, E. Torró Pastor ${ }^{168}$, J. $\operatorname{Toth}^{84, a g}$, F. Touchard ${ }^{84}$,

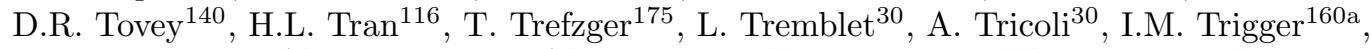
S. Trincaz-Duvoid ${ }^{79}$, M.F. Tripiana ${ }^{70}$, N. Triplett ${ }^{25}$, W. Trischuk ${ }^{159}$, B. Trocmé55, C. Troncon ${ }^{90 a}$, M. Trottier-McDonald ${ }^{143}$, M. Trovatelli ${ }^{135 a, 135 b}$, P. True ${ }^{89}$, M. Trzebinski ${ }^{39}$, A. Trzupek ${ }^{39}$, C. Tsarouchas ${ }^{30}$, J.C-L. Tseng ${ }^{119}$, P.V. Tsiareshka ${ }^{91}$, D. Tsionou ${ }^{137}$, G. Tsipolitis ${ }^{10}$,

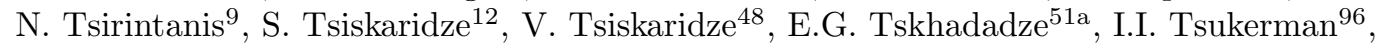
V. Tsulaia ${ }^{15}$, J.-W. Tsung ${ }^{21}$, S. Tsuno ${ }^{65}$, D. Tsybychev ${ }^{149}$, A. Tua ${ }^{140}$, A. Tudorache ${ }^{26 a}$, V. Tudorache ${ }^{26 a}$, J.M. Tuggle ${ }^{31}$, A.N. Tuna ${ }^{121}$, S.A. Tupputi ${ }^{20 a, 20 b}$, S. Turchikhin ${ }^{98, a k}$, D. Turecek ${ }^{127}$, I. Turk Cakir ${ }^{4 d}$, R. Turra90a,90b, P.M. Tuts ${ }^{35}$, A. Tykhonov ${ }^{74}$, M. Tylmad ${ }^{147 a, 147 b}$, M. Tyndel ${ }^{130}$, K. Uchida ${ }^{21}$, I. Ueda ${ }^{156}$, R. Ueno ${ }^{29}$, M. Ughetto ${ }^{84}$, M. Ugland ${ }^{14}$, M. Uhlenbrock ${ }^{21}$,

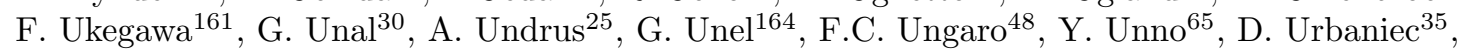
P. Urquijo ${ }^{21}$, G. Usai ${ }^{8}$, A. Usanova ${ }^{61}$, L. Vacavant ${ }^{84}$, V. Vacek ${ }^{127}$, B. Vachon ${ }^{86}$, S. Vahsen $^{15}$, N. Valencic ${ }^{106}$, S. Valentinetti ${ }^{20 a, 20 b}$, A. Valero ${ }^{168}$, L. Valery $^{34}$, S. Valkar $^{128}$,

E. Valladolid Gallego ${ }^{168}$, S. Vallecorsa ${ }^{49}$, J.A. Valls Ferrer ${ }^{168}$, R. Van Berg ${ }^{121}$,

P.C. Van Der Deijl ${ }^{106}$, R. van der Geer ${ }^{106}$, H. van der Graaf ${ }^{106}$, R. Van Der Leeuw ${ }^{106}$, D. van der $\operatorname{Ster}^{30}$, N. van Eldik ${ }^{30}$, P. van Gemmeren ${ }^{6}$, J. Van Nieuwkoop ${ }^{143}$, I. van Vulpen ${ }^{106}$, 


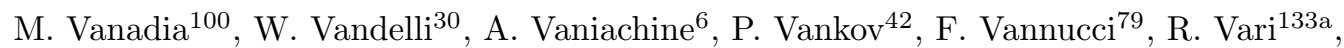
E.W. Varnes ${ }^{7}$, T. Varol $^{85}$, D. Varouchas ${ }^{15}$, A. Vartapetian ${ }^{8}$, K.E. Varvell ${ }^{151}$,

V.I. Vassilakopoulos ${ }^{56}$, F. Vazeille ${ }^{34}$, T. Vazquez Schroeder ${ }^{54}$, J. Veatch ${ }^{7}$, F. Veloso ${ }^{125 a}$, S. Veneziano ${ }^{133 a}$, A. Ventura ${ }^{72 a}, 72 b$, D. Ventura ${ }^{85}$, M. Venturi ${ }^{48}$, N. Venturi ${ }^{159}, V^{14}$. Vercesi ${ }^{120 a}$, M. Verducci ${ }^{139}$, W. Verkerke ${ }^{106}$, J.C. Vermeulen ${ }^{106}$, A. Vest ${ }^{44}$, M.C. Vetterli ${ }^{143, f}$, O. Viazlo ${ }^{80}$, I. Vichou ${ }^{166}$, T. Vickey ${ }^{146 c, a n}$, O.E. Vickey Boeriu ${ }^{146 c}$, G.H.A. Viehhauser ${ }^{119}$, S. Viel ${ }^{169}$, R. Vigne ${ }^{30}$, M. Villa ${ }^{20 a}, 20 \mathrm{~b}$, M. Villaplana Perez ${ }^{168}$, E. Vilucchi ${ }^{47}$, M.G. Vincter ${ }^{29}$, V.B. Vinogradov ${ }^{64}$, J. Virzi ${ }^{15}$, O. Vitells ${ }^{173}$, M. Viti ${ }^{42}$, I. Vivarelli ${ }^{150}$, F. Vives Vaque ${ }^{3}$, S. Vlachos ${ }^{10}$, D. Vladoiu ${ }^{99}$, M. Vlasak ${ }^{127}$, A. Vogel ${ }^{21}$, P. Vokac $^{127}$, G. Volpi ${ }^{47}$, M. Volpi ${ }^{87}$, G. Volpini ${ }^{90 a}$, H. von der Schmitt ${ }^{100}$, H. von Radziewski ${ }^{48}$, E. von Toerne ${ }^{21}$, V. Vorobel ${ }^{128}$, M. Vos ${ }^{168}$, R. $\operatorname{Voss}^{30}$, J.H. Vossebeld ${ }^{73}$, N. Vranjes ${ }^{137}$, M. Vranjes Milosavljevic ${ }^{106}$, V. Vrba ${ }^{126}$, M. Vreeswijk ${ }^{106}$, T. Vu $\mathrm{Anh}^{48}$, R. Vuillermet ${ }^{30}$, I. Vukotic ${ }^{31}$, Z. Vykydal ${ }^{127}$, W. Wagner ${ }^{176}$, P. Wagner ${ }^{21}$, S. Wahrmund ${ }^{44}$, J. Wakabayashi ${ }^{102}$, S. Walch ${ }^{88}$, J. Walder ${ }^{71}$, R. Walker ${ }^{99}$, W. Walkowiak ${ }^{142}$, R. Wall ${ }^{177}$, P. Waller ${ }^{73}$, B. Walsh ${ }^{177}$, C. Wang ${ }^{45}$, H. Wang ${ }^{174}$, H. Wang ${ }^{40}$, J. Wang ${ }^{152}$, J. Wang ${ }^{33 a}$, K. Wang ${ }^{86}$, R. Wang ${ }^{104}$, S.M. Wang ${ }^{152}$, T. Wang ${ }^{21}$, X. Wang ${ }^{177}$, A. Warburton ${ }^{86}$, C.P. Ward ${ }^{28}$, D.R. Wardrope ${ }^{77}$, M. Warsinsky ${ }^{48}$, A. Washbrook ${ }^{46}$, C. Wasicki ${ }^{42}$, I. Watanabe ${ }^{66}$, P.M. Watkins ${ }^{18}$, A.T. Watson ${ }^{18}$, I.J. Watson ${ }^{151}$, M.F. Watson ${ }^{18}$, G. Watts ${ }^{139}$, S. Watts ${ }^{83}$, A.T. Waugh ${ }^{151}$, B.M. Waugh ${ }^{77}$, S. Webb ${ }^{83}$, M.S. Weber ${ }^{17}$, S.W. Weber ${ }^{175}$, J.S. Webster ${ }^{31}$, A.R. Weidberg ${ }^{119}$, P. Weigell ${ }^{100}$, J. Weingarten ${ }^{54}$, C. Weiser ${ }^{48}$, H. Weits ${ }^{106}$, P.S. Wells ${ }^{30}$, T. Wenaus ${ }^{25}$, D. Wendland ${ }^{16}$, Z. Weng ${ }^{152, x}$, T. Wengler ${ }^{30}$, S. Wenig ${ }^{30}$, N. Wermes ${ }^{21}$, M. Werner ${ }^{48}$, P. Werner ${ }^{30}$, M. Werth ${ }^{164}$, M. Wessels ${ }^{58 a}$, J. Wetter ${ }^{162}$, K. Whalen ${ }^{29}$, A. White ${ }^{8}$, M.J. White ${ }^{1}$, R. White ${ }^{32 b}$, S. White ${ }^{123 a, 123 b}$, D. Whiteson ${ }^{164}$, D. Whittington ${ }^{60}$, D. Wicke ${ }^{176}$, F.J. Wickens ${ }^{130}$, W. Wiedenmann ${ }^{174}$, M. Wielers ${ }^{80, e}$, P. Wienemann $^{21}$, C. Wiglesworth ${ }^{36}$, L.A.M. Wiik-Fuchs ${ }^{21}$, P.A. Wijeratne ${ }^{77}$, A. Wildauer ${ }^{100}$, M.A. Wildt ${ }^{42, a o}$, I. Wilhelm ${ }^{128}$, H.G. Wilkens ${ }^{30}$, J.Z. Will ${ }^{99}$, E. Williams ${ }^{35}$, H.H. Williams ${ }^{121}$, S. Williams ${ }^{28}$, W. Willis ${ }^{35, *}$, S. Willocq ${ }^{85}$, J.A. Wilson ${ }^{18}$, A. Wilson ${ }^{88}$, I. Wingerter-Seez ${ }^{5}$, S. Winkelmann ${ }^{48}$, F. Winklmeier ${ }^{115}$, M. Wittgen ${ }^{144}$, T. Wittig ${ }^{43}$, J. Wittkowski ${ }^{99}$, S.J. Wollstadt ${ }^{82}$, M.W. Wolter ${ }^{39}$, H. Wolters ${ }^{125 a, i}$, W.C. Wong ${ }^{41}$, B.K. Wosiek ${ }^{39}$, J. Wotschack ${ }^{30}$, M.J. Woudstra ${ }^{83}$, K.W. Wozniak ${ }^{39}$, K. Wraight ${ }^{53}$, M. Wright ${ }^{53}$, S.L. $\mathrm{Wu}^{174}$, X. Wu ${ }^{49}$, Y. Wu ${ }^{88}$, E. Wulf ${ }^{35}$, T.R. Wyatt ${ }^{83}$, B.M. Wynne ${ }^{46}$, S. Xella ${ }^{36}$, M. Xiao ${ }^{137}$, C. Xu ${ }^{33 b, a p}$, D. Xu ${ }^{33 a}$, L. Xu ${ }^{33 b, a q}$, B. Yabsley ${ }^{151}$, S. Yacoob ${ }^{146 b, a r}$, M. Yamada ${ }^{65}$, H. Yamaguchi ${ }^{156}$, Y. Yamaguchi ${ }^{156}$, A. Yamamoto ${ }^{65}$, K. Yamamoto $^{63}$, S. Yamamoto ${ }^{156}$,

T. Yamamura ${ }^{156}$, T. Yamanaka ${ }^{156}$, K. Yamauchi ${ }^{102}$, Y. Yamazaki ${ }^{66}$, Z. Yan ${ }^{22}$, H. Yang ${ }^{33 e}$, H. Yang ${ }^{174}$, U.K. Yang ${ }^{83}$, Y. Yang ${ }^{110}$, Z. Yang ${ }^{147 a, 147 b}$, S. Yanush ${ }^{92}$, L. Yao ${ }^{33 a}$, Y. Yasu ${ }^{65}$, E. Yatsenko ${ }^{42}$, K.H. Yau Wong ${ }^{21}$, J. Ye ${ }^{40}$, S. Ye ${ }^{25}$, A.L. Yen ${ }^{57}$, E. Yildirim ${ }^{42}$, M. Yilmaz ${ }^{4 b}$, R. Yoosoofmiya ${ }^{124}$, K. Yorita ${ }^{172}$, R. Yoshida ${ }^{6}$, K. Yoshihara ${ }^{156}$, C. Young ${ }^{144}$, C.J.S. Young ${ }^{30}$, S. Youssef ${ }^{22}$, D.R. $\mathrm{Yu}^{15}$, J. $\mathrm{Yu}^{8}$, J. Yu ${ }^{113}$, L. Yuan ${ }^{66}$, A. Yurkewicz ${ }^{107}$, B. Zabinski ${ }^{39}$, R. Zaidan ${ }^{62}$, A.M. Zaitsev ${ }^{129, a c}$, A. Zaman ${ }^{149}$, S. Zambito ${ }^{23}$, L. Zanello ${ }^{133 a, 133 b}$, D. Zanzi ${ }^{100}$, A. Zaytsev ${ }^{25}$,

C. Zeitnitz ${ }^{176}$, M. Zeman ${ }^{127}$, A. Zemla ${ }^{38 a}$, O. Zenin ${ }^{129}$, T. Ženišs ${ }^{145 a}$, D. Zerwas ${ }^{116}$,

G. Zevi della Porta ${ }^{57}$, D. Zhang ${ }^{88}$, H. Zhang ${ }^{89}$, J. Zhang ${ }^{6}$, L. Zhang ${ }^{152}$, X. Zhang ${ }^{33 d}$, Z. Zhang ${ }^{116}$, Z. Zhao ${ }^{33 b}$, A. Zhemchugov ${ }^{64}$, J. Zhong ${ }^{119}$, B. Zhou ${ }^{88}$, L. Zhou ${ }^{35}$, N. Zhou ${ }^{164}$, C.G. Zhu ${ }^{33 d}$, H. Zhu ${ }^{42}$, J. Zhu ${ }^{88}$, Y. Zhu ${ }^{33 b}$, X. Zhuang ${ }^{33 a}$, A. Zibell ${ }^{99}$, D. Zieminska ${ }^{60}$, N.I. Zimin ${ }^{64}$,

C. Zimmermann ${ }^{82}$, R. Zimmermann ${ }^{21}$, S. Zimmermann ${ }^{21}$, S. Zimmermann ${ }^{48}$, Z. Zinonos ${ }^{123 a, 123 b}$,

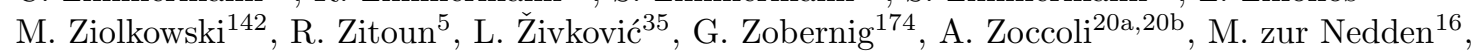
G. Zurzolo ${ }^{103 a, 103 b}$, V. Zutshi ${ }^{107}$, L. Zwalinski ${ }^{30}$

1 School of Chemistry and Physics, University of Adelaide, Adelaide, Australia

2 Physics Department, SUNY Albany, Albany NY, United States of America

3 Department of Physics, University of Alberta, Edmonton AB, Canada

4 (a) Department of Physics, Ankara University, Ankara; ${ }^{(b)}$ Department of Physics, Gazi University, Ankara; ${ }^{(c)}$ Division of Physics, TOBB University of Economics and Technology, Ankara; ${ }^{(d)}$ Turkish Atomic Energy Authority, Ankara, Turkey

5 LAPP, CNRS/IN2P3 and Université de Savoie, Annecy-le-Vieux, France

6 High Energy Physics Division, Argonne National Laboratory, Argonne IL, United States of America 
7 Department of Physics, University of Arizona, Tucson AZ, United States of America

8 Department of Physics, The University of Texas at Arlington, Arlington TX, United States of America

9 Physics Department, University of Athens, Athens, Greece

10 Physics Department, National Technical University of Athens, Zografou, Greece

11 Institute of Physics, Azerbaijan Academy of Sciences, Baku, Azerbaijan

12 Institut de Física d'Altes Energies and Departament de Física de la Universitat Autònoma de Barcelona, Barcelona, Spain

13 (a) Institute of Physics, University of Belgrade, Belgrade; ${ }^{(b)}$ Vinca Institute of Nuclear Sciences, University of Belgrade, Belgrade, Serbia

14 Department for Physics and Technology, University of Bergen, Bergen, Norway

15 Physics Division, Lawrence Berkeley National Laboratory and University of California, Berkeley CA, United States of America

16 Department of Physics, Humboldt University, Berlin, Germany

17 Albert Einstein Center for Fundamental Physics and Laboratory for High Energy Physics, University of Bern, Bern, Switzerland

18 School of Physics and Astronomy, University of Birmingham, Birmingham, United Kingdom

19 (a) Department of Physics, Bogazici University, Istanbul; ${ }^{(b)}$ Department of Physics, Dogus University, Istanbul; ${ }^{(c)}$ Department of Physics Engineering, Gaziantep University, Gaziantep, Turkey

20 (a) INFN Sezione di Bologna; ${ }^{(b)}$ Dipartimento di Fisica e Astronomia, Università di Bologna, Bologna, Italy

21 Physikalisches Institut, University of Bonn, Bonn, Germany

22 Department of Physics, Boston University, Boston MA, United States of America

23 Department of Physics, Brandeis University, Waltham MA, United States of America

24 (a) Universidade Federal do Rio De Janeiro COPPE/EE/IF, Rio de Janeiro; ${ }^{(b)}$ Federal University of Juiz de Fora (UFJF), Juiz de Fora; ${ }^{(c)}$ Federal University of Sao Joao del Rei (UFSJ), Sao Joao del Rei; ${ }^{(d)}$ Instituto de Fisica, Universidade de Sao Paulo, Sao Paulo, Brazil

25 Physics Department, Brookhaven National Laboratory, Upton NY, United States of America

26 (a) National Institute of Physics and Nuclear Engineering, Bucharest; ${ }^{(b)}$ National Institute for Research and Development of Isotopic and Molecular Technologies, Physics Department, Cluj Napoca; ${ }^{(c)}$ University Politehnica Bucharest, Bucharest; ${ }^{(d)}$ West University in Timisoara, Timisoara, Romania

27 Departamento de Física, Universidad de Buenos Aires, Buenos Aires, Argentina

28 Cavendish Laboratory, University of Cambridge, Cambridge, United Kingdom

29 Department of Physics, Carleton University, Ottawa ON, Canada

30 CERN, Geneva, Switzerland

31 Enrico Fermi Institute, University of Chicago, Chicago IL, United States of America

$32{ }^{(a)}$ Departamento de Física, Pontificia Universidad Católica de Chile, Santiago; ${ }^{(b)}$ Departamento de Física, Universidad Técnica Federico Santa María, Valparaíso, Chile

33 (a) Institute of High Energy Physics, Chinese Academy of Sciences, Beijing; ${ }^{(b)}$ Department of Modern Physics, University of Science and Technology of China, Anhui; ${ }^{(c)}$ Department of Physics, Nanjing University, Jiangsu; ${ }^{(d)}$ School of Physics, Shandong University, Shandong; ${ }^{(e)}$ Physics Department, Shanghai Jiao Tong University, Shanghai, China

34 Laboratoire de Physique Corpusculaire, Clermont Université and Université Blaise Pascal and CNRS/IN2P3, Clermont-Ferrand, France

35 Nevis Laboratory, Columbia University, Irvington NY, United States of America

36 Niels Bohr Institute, University of Copenhagen, Kobenhavn, Denmark

37 (a) INFN Gruppo Collegato di Cosenza; ${ }^{(b)}$ Dipartimento di Fisica, Università della Calabria, Rende, Italy

38 (a) AGH University of Science and Technology, Faculty of Physics and Applied Computer Science, Krakow; ${ }^{(b)}$ Marian Smoluchowski Institute of Physics, Jagiellonian University, Krakow, Poland 
The Henryk Niewodniczanski Institute of Nuclear Physics, Polish Academy of Sciences, Krakow, Poland

40 Physics Department, Southern Methodist University, Dallas TX, United States of America

${ }^{41}$ Physics Department, University of Texas at Dallas, Richardson TX, United States of America

42 DESY, Hamburg and Zeuthen, Germany

43 Institut für Experimentelle Physik IV, Technische Universität Dortmund, Dortmund, Germany

${ }^{44}$ Institut für Kern- und Teilchenphysik, Technische Universität Dresden, Dresden, Germany

45 Department of Physics, Duke University, Durham NC, United States of America

${ }^{46}$ SUPA - School of Physics and Astronomy, University of Edinburgh, Edinburgh, United Kingdom

47 INFN Laboratori Nazionali di Frascati, Frascati, Italy

48 Fakultät für Mathematik und Physik, Albert-Ludwigs-Universität, Freiburg, Germany

49 Section de Physique, Université de Genève, Geneva, Switzerland

50 (a) INFN Sezione di Genova; ${ }^{(b)}$ Dipartimento di Fisica, Università di Genova, Genova, Italy

51 (a) E. Andronikashvili Institute of Physics, Iv. Javakhishvili Tbilisi State University, Tbilisi; ${ }^{(b)}$

High Energy Physics Institute, Tbilisi State University, Tbilisi, Georgia

52 II Physikalisches Institut, Justus-Liebig-Universität Giessen, Giessen, Germany

${ }^{53}$ SUPA - School of Physics and Astronomy, University of Glasgow, Glasgow, United Kingdom

54 II Physikalisches Institut, Georg-August-Universität, Göttingen, Germany

55 Laboratoire de Physique Subatomique et de Cosmologie, Université Joseph Fourier and CNRS/IN2P3 and Institut National Polytechnique de Grenoble, Grenoble, France

56 Department of Physics, Hampton University, Hampton VA, United States of America

57 Laboratory for Particle Physics and Cosmology, Harvard University, Cambridge MA, United States of America

58 (a) Kirchhoff-Institut für Physik, Ruprecht-Karls-Universität Heidelberg, Heidelberg; ${ }^{(b)}$ Physikalisches Institut, Ruprecht-Karls-Universität Heidelberg, Heidelberg; ${ }^{(c)}$ ZITI Institut für technische Informatik, Ruprecht-Karls-Universität Heidelberg, Mannheim, Germany

59 Faculty of Applied Information Science, Hiroshima Institute of Technology, Hiroshima, Japan

60 Department of Physics, Indiana University, Bloomington IN, United States of America

${ }^{61}$ Institut für Astro- und Teilchenphysik, Leopold-Franzens-Universität, Innsbruck, Austria

62 University of Iowa, Iowa City IA, United States of America

63 Department of Physics and Astronomy, Iowa State University, Ames IA, United States of America

${ }^{64}$ Joint Institute for Nuclear Research, JINR Dubna, Dubna, Russia

${ }^{65}$ KEK, High Energy Accelerator Research Organization, Tsukuba, Japan

${ }^{66}$ Graduate School of Science, Kobe University, Kobe, Japan

${ }^{67}$ Faculty of Science, Kyoto University, Kyoto, Japan

68 Kyoto University of Education, Kyoto, Japan

69 Department of Physics, Kyushu University, Fukuoka, Japan

${ }^{70}$ Instituto de Física La Plata, Universidad Nacional de La Plata and CONICET, La Plata, Argentina

71 Physics Department, Lancaster University, Lancaster, United Kingdom

72 (a) INFN Sezione di Lecce; ${ }^{(b)}$ Dipartimento di Matematica e Fisica, Università del Salento, Lecce, Italy

${ }^{73}$ Oliver Lodge Laboratory, University of Liverpool, Liverpool, United Kingdom

74 Department of Physics, Jožef Stefan Institute and University of Ljubljana, Ljubljana, Slovenia

75 School of Physics and Astronomy, Queen Mary University of London, London, United Kingdom

76 Department of Physics, Royal Holloway University of London, Surrey, United Kingdom

77 Department of Physics and Astronomy, University College London, London, United Kingdom

78 Louisiana Tech University, Ruston LA, United States of America

79 Laboratoire de Physique Nucléaire et de Hautes Energies, UPMC and Université Paris-Diderot and CNRS/IN2P3, Paris, France

80 Fysiska institutionen, Lunds universitet, Lund, Sweden

81 Departamento de Fisica Teorica C-15, Universidad Autonoma de Madrid, Madrid, Spain

82 Institut für Physik, Universität Mainz, Mainz, Germany 
School of Physics and Astronomy, University of Manchester, Manchester, United Kingdom

${ }^{84}$ CPPM, Aix-Marseille Université and CNRS/IN2P3, Marseille, France

85 Department of Physics, University of Massachusetts, Amherst MA, United States of America

86 Department of Physics, McGill University, Montreal QC, Canada

87 School of Physics, University of Melbourne, Victoria, Australia

88 Department of Physics, The University of Michigan, Ann Arbor MI, United States of America

${ }^{89}$ Department of Physics and Astronomy, Michigan State University, East Lansing MI, United States of America

90 (a) INFN Sezione di Milano; ${ }^{(b)}$ Dipartimento di Fisica, Università di Milano, Milano, Italy

91 B.I. Stepanov Institute of Physics, National Academy of Sciences of Belarus, Minsk, Republic of Belarus

92 National Scientific and Educational Centre for Particle and High Energy Physics, Minsk, Republic of Belarus

93 Department of Physics, Massachusetts Institute of Technology, Cambridge MA, United States of America

94 Group of Particle Physics, University of Montreal, Montreal QC, Canada

${ }^{9}$ P.N. Lebedev Institute of Physics, Academy of Sciences, Moscow, Russia

96 Institute for Theoretical and Experimental Physics (ITEP), Moscow, Russia

97 Moscow Engineering and Physics Institute (MEPhI), Moscow, Russia

98 D.V.Skobeltsyn Institute of Nuclear Physics, M.V.Lomonosov Moscow State University, Moscow, Russia

99 Fakultät für Physik, Ludwig-Maximilians-Universität München, München, Germany

100 Max-Planck-Institut für Physik (Werner-Heisenberg-Institut), München, Germany

101 Nagasaki Institute of Applied Science, Nagasaki, Japan

102 Graduate School of Science and Kobayashi-Maskawa Institute, Nagoya University, Nagoya, Japan

103 (a) INFN Sezione di Napoli; ${ }^{(b)}$ Dipartimento di Scienze Fisiche, Università di Napoli, Napoli, Italy

104 Department of Physics and Astronomy, University of New Mexico, Albuquerque NM, United States of America

105 Institute for Mathematics, Astrophysics and Particle Physics, Radboud University Nijmegen/Nikhef, Nijmegen, Netherlands

106 Nikhef National Institute for Subatomic Physics and University of Amsterdam, Amsterdam, Netherlands

107 Department of Physics, Northern Illinois University, DeKalb IL, United States of America

108 Budker Institute of Nuclear Physics, SB RAS, Novosibirsk, Russia

109 Department of Physics, New York University, New York NY, United States of America

110 Ohio State University, Columbus OH, United States of America

111 Faculty of Science, Okayama University, Okayama, Japan

112 Homer L. Dodge Department of Physics and Astronomy, University of Oklahoma, Norman OK, United States of America

113 Department of Physics, Oklahoma State University, Stillwater OK, United States of America

114 Palacký University, RCPTM, Olomouc, Czech Republic

115 Center for High Energy Physics, University of Oregon, Eugene OR, United States of America

116 LAL, Université Paris-Sud and CNRS/IN2P3, Orsay, France

117 Graduate School of Science, Osaka University, Osaka, Japan

118 Department of Physics, University of Oslo, Oslo, Norway

119 Department of Physics, Oxford University, Oxford, United Kingdom

120 (a) INFN Sezione di Pavia; ${ }^{(b)}$ Dipartimento di Fisica, Università di Pavia, Pavia, Italy

121 Department of Physics, University of Pennsylvania, Philadelphia PA, United States of America

122 Petersburg Nuclear Physics Institute, Gatchina, Russia

123 (a) INFN Sezione di Pisa; ${ }^{(b)}$ Dipartimento di Fisica E. Fermi, Università di Pisa, Pisa, Italy

124 Department of Physics and Astronomy, University of Pittsburgh, Pittsburgh PA, United States of America 

Departamento de Fisica Teorica y del Cosmos and CAFPE, Universidad de Granada, Granada, Spain

126 Institute of Physics, Academy of Sciences of the Czech Republic, Praha, Czech Republic

127 Czech Technical University in Prague, Praha, Czech Republic

128 Faculty of Mathematics and Physics, Charles University in Prague, Praha, Czech Republic

129 State Research Center Institute for High Energy Physics, Protvino, Russia

130 Particle Physics Department, Rutherford Appleton Laboratory, Didcot, United Kingdom

131 Physics Department, University of Regina, Regina SK, Canada

132 Ritsumeikan University, Kusatsu, Shiga, Japan

133 (a) INFN Sezione di Roma I; ${ }^{(b)}$ Dipartimento di Fisica, Università La Sapienza, Roma, Italy

134 (a) INFN Sezione di Roma Tor Vergata; ${ }^{(b)}$ Dipartimento di Fisica, Università di Roma Tor Vergata, Roma, Italy

135 (a) INFN Sezione di Roma Tre; ${ }^{(b)}$ Dipartimento di Matematica e Fisica, Università Roma Tre, Roma, Italy

136 (a) Faculté des Sciences Ain Chock, Réseau Universitaire de Physique des Hautes Energies Université Hassan II, Casablanca; ${ }^{(b)}$ Centre National de l'Energie des Sciences Techniques Nucleaires, Rabat; ${ }^{(c)}$ Faculté des Sciences Semlalia, Université Cadi Ayyad, LPHEA-Marrakech; ${ }^{(d)}$ Faculté des Sciences, Université Mohamed Premier and LPTPM, Oujda; ${ }^{(e)}$ Faculté des sciences, Université Mohammed V-Agdal, Rabat, Morocco

137 DSM/IRFU (Institut de Recherches sur les Lois Fondamentales de l'Univers), CEA Saclay (Commissariat à l'Energie Atomique et aux Energies Alternatives), Gif-sur-Yvette, France

138 Santa Cruz Institute for Particle Physics, University of California Santa Cruz, Santa Cruz CA, United States of America

139 Department of Physics, University of Washington, Seattle WA, United States of America

140 Department of Physics and Astronomy, University of Sheffield, Sheffield, United Kingdom

141 Department of Physics, Shinshu University, Nagano, Japan

142 Fachbereich Physik, Universität Siegen, Siegen, Germany

143 Department of Physics, Simon Fraser University, Burnaby BC, Canada

144 SLAC National Accelerator Laboratory, Stanford CA, United States of America

$145{ }^{(a)}$ Faculty of Mathematics, Physics \& Informatics, Comenius University, Bratislava; ${ }^{(b)}$

Department of Subnuclear Physics, Institute of Experimental Physics of the Slovak Academy of Sciences, Kosice, Slovak Republic

146 (a) Department of Physics, University of Cape Town, Cape Town; ${ }^{(b)}$ Department of Physics, University of Johannesburg, Johannesburg; (c) School of Physics, University of the Witwatersrand, Johannesburg, South Africa

147 (a) Department of Physics, Stockholm University; ${ }^{(b)}$ The Oskar Klein Centre, Stockholm, Sweden 148 Physics Department, Royal Institute of Technology, Stockholm, Sweden

149 Departments of Physics \& Astronomy and Chemistry, Stony Brook University, Stony Brook NY, United States of America

150 Department of Physics and Astronomy, University of Sussex, Brighton, United Kingdom

151 School of Physics, University of Sydney, Sydney, Australia

152 Institute of Physics, Academia Sinica, Taipei, Taiwan

153 Department of Physics, Technion: Israel Institute of Technology, Haifa, Israel

154 Raymond and Beverly Sackler School of Physics and Astronomy, Tel Aviv University, Tel Aviv, Israel

155 Department of Physics, Aristotle University of Thessaloniki, Thessaloniki, Greece

156 International Center for Elementary Particle Physics and Department of Physics, The University of Tokyo, Tokyo, Japan

157 Graduate School of Science and Technology, Tokyo Metropolitan University, Tokyo, Japan

158 Department of Physics, Tokyo Institute of Technology, Tokyo, Japan

159 Department of Physics, University of Toronto, Toronto ON, Canada 
160 (a) TRIUMF, Vancouver BC; ${ }^{(b)}$ Department of Physics and Astronomy, York University, Toronto ON, Canada

161 Faculty of Pure and Applied Sciences, University of Tsukuba, Tsukuba, Japan

162 Department of Physics and Astronomy, Tufts University, Medford MA, United States of America

163 Centro de Investigaciones, Universidad Antonio Narino, Bogota, Colombia

164 Department of Physics and Astronomy, University of California Irvine, Irvine CA, United States of America

165 (a) INFN Gruppo Collegato di Udine; ${ }^{(b)}$ ICTP, Trieste; ${ }^{(c)}$ Dipartimento di Chimica, Fisica e Ambiente, Università di Udine, Udine, Italy

166 Department of Physics, University of Illinois, Urbana IL, United States of America

167 Department of Physics and Astronomy, University of Uppsala, Uppsala, Sweden

168 Instituto de Física Corpuscular (IFIC) and Departamento de Física Atómica, Molecular y Nuclear and Departamento de Ingeniería Electrónica and Instituto de Microelectrónica de Barcelona (IMB-CNM), University of Valencia and CSIC, Valencia, Spain

169 Department of Physics, University of British Columbia, Vancouver BC, Canada

170 Department of Physics and Astronomy, University of Victoria, Victoria BC, Canada

171 Department of Physics, University of Warwick, Coventry, United Kingdom

172 Waseda University, Tokyo, Japan

173 Department of Particle Physics, The Weizmann Institute of Science, Rehovot, Israel

174 Department of Physics, University of Wisconsin, Madison WI, United States of America

175 Fakultät für Physik und Astronomie, Julius-Maximilians-Universität, Würzburg, Germany

176 Fachbereich C Physik, Bergische Universität Wuppertal, Wuppertal, Germany

177 Department of Physics, Yale University, New Haven CT, United States of America

178 Yerevan Physics Institute, Yerevan, Armenia

179 Centre de Calcul de l'Institut National de Physique Nucléaire et de Physique des Particules (IN2P3), Villeurbanne, France

a Also at Department of Physics, King's College London, London, United Kingdom

$b$ Also at Laboratorio de Instrumentacao e Fisica Experimental de Particulas - LIP, Lisboa, Portugal

c Also at Institute of Physics, Azerbaijan Academy of Sciences, Baku, Azerbaijan

$d$ Also at Faculdade de Ciencias and CFNUL, Universidade de Lisboa, Lisboa, Portugal

e Also at Particle Physics Department, Rutherford Appleton Laboratory, Didcot, United Kingdom

$f$ Also at TRIUMF, Vancouver BC, Canada

$g$ Also at Department of Physics, California State University, Fresno CA, United States of America

$h$ Also at Novosibirsk State University, Novosibirsk, Russia

${ }^{i}$ Also at Department of Physics, University of Coimbra, Coimbra, Portugal

$j$ Also at Università di Napoli Parthenope, Napoli, Italy

$k$ Also at Institute of Particle Physics (IPP), Canada

$l$ Also at Department of Physics, Middle East Technical University, Ankara, Turkey

$m$ Also at Louisiana Tech University, Ruston LA, United States of America

$n$ Also at Dep Fisica and CEFITEC of Faculdade de Ciencias e Tecnologia, Universidade Nova de Lisboa, Caparica, Portugal

o Also at CPPM, Aix-Marseille Université and CNRS/IN2P3, Marseille, France

$p$ Also at Department of Physics and Astronomy, Michigan State University, East Lansing MI, United States of America

$q$ Also at Department of Financial and Management Engineering, University of the Aegean, Chios, Greece

$r$ Also at Institucio Catalana de Recerca i Estudis Avancats, ICREA, Barcelona, Spain

$s$ Also at Department of Physics, University of Cape Town, Cape Town, South Africa

${ }^{t}$ Also at CERN, Geneva, Switzerland

u Also at Ochadai Academic Production, Ochanomizu University, Tokyo, Japan

$v$ Also at Manhattan College, New York NY, United States of America 
w Also at Institute of Physics, Academia Sinica, Taipei, Taiwan

$x$ Also at School of Physics and Engineering, Sun Yat-sen University, Guanzhou, China

y Also at Academia Sinica Grid Computing, Institute of Physics, Academia Sinica, Taipei, Taiwan

$z$ Also at Laboratoire de Physique Nucléaire et de Hautes Energies, UPMC and Université Paris-Diderot and CNRS/IN2P3, Paris, France

aa Also at School of Physical Sciences, National Institute of Science Education and Research, Bhubaneswar, India

$a b$ Also at Dipartimento di Fisica, Università La Sapienza, Roma, Italy

ac Also at Moscow Institute of Physics and Technology State University, Dolgoprudny, Russia

ad Also at Section de Physique, Université de Genève, Geneva, Switzerland

ae Also at Departamento de Fisica, Universidade de Minho, Braga, Portugal

af Also at Department of Physics, The University of Texas at Austin, Austin TX, United States of America

ag Also at Institute for Particle and Nuclear Physics, Wigner Research Centre for Physics, Budapest, Hungary

ah Also at DESY, Hamburg and Zeuthen, Germany

ai Also at International School for Advanced Studies (SISSA), Trieste, Italy

aj Also at Department of Physics and Astronomy, University of South Carolina, Columbia SC, United States of America

ak Also at Faculty of Physics, M.V.Lomonosov Moscow State University, Moscow, Russia

al Also at Physics Department, Brookhaven National Laboratory, Upton NY, United States of America

am Also at Moscow Engineering and Physics Institute (MEPhI), Moscow, Russia

an Also at Department of Physics, Oxford University, Oxford, United Kingdom

ao Also at Institut für Experimentalphysik, Universität Hamburg, Hamburg, Germany

ap Also at DSM/IRFU (Institut de Recherches sur les Lois Fondamentales de l'Univers), CEA Saclay (Commissariat à l'Energie Atomique et aux Energies Alternatives), Gif-sur-Yvette, France

aq Also at Department of Physics, The University of Michigan, Ann Arbor MI, United States of America

ar Also at Discipline of Physics, University of KwaZulu-Natal, Durban, South Africa

* Deceased 